Network Working Group

Request for Comments: 4306

Obsoletes: 2407,2408, 2409

Category: Standards Track
C. Kaufman, Ed. Microsoft

December 2005

\title{
Internet Key Exchange (IKEv2) Protocol
}

Status of This Memo

This document specifies an Internet standards track protocol for the Internet community, and requests discussion and suggestions for improvements. Please refer to the current edition of the "Internet Official Protocol Standards" (STD 1) for the standardization state and status of this protocol. Distribution of this memo is unlimited.

Copyright Notice

Copyright (C) The Internet Society (2005) .

Abstract

This document describes version 2 of the Internet Key Exchange (IKE) protocol. IKE is a component of IPsec used for performing mutual authentication and establishing and maintaining security associations (SAs) .

This version of the IKE specification combines the contents of what were previously separate documents, including Internet security Association and Key Management Protocol (ISAKMP, RFC 2408), IKE (RFC 2409), the Internet Domain of Interpretation (DOI, RFC 2407), Network Address Translation (NAT) Traversal, Legacy authentication, and remote address acquisition.

Version 2 of IKE does not interoperate with version 1, but it has enough of the header format in common that both versions can unambiguously run over the same UDP port. 
Table of Contents

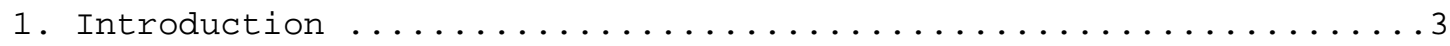

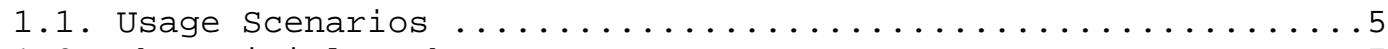

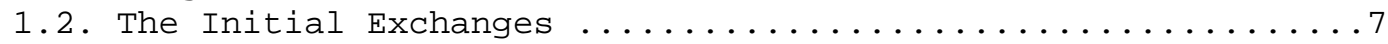

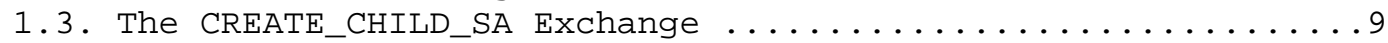

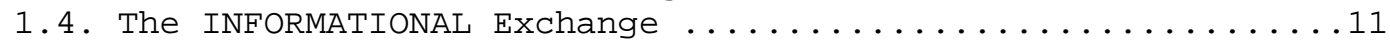

1.5. Informational Messages outside of an IKE_SA ..........12

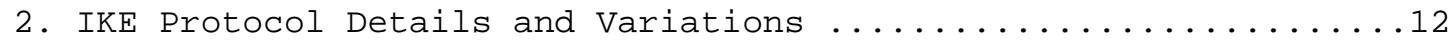

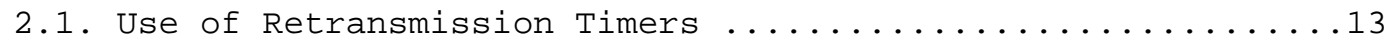

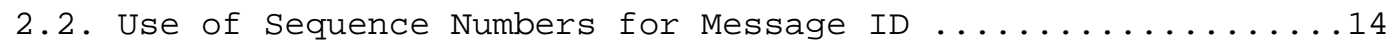

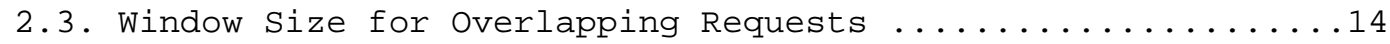

2.4. State Synchronization and Connection Timeouts .........15

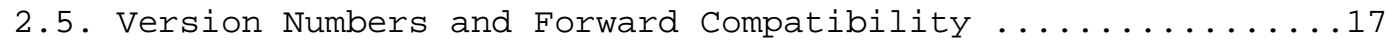

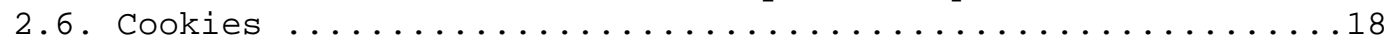

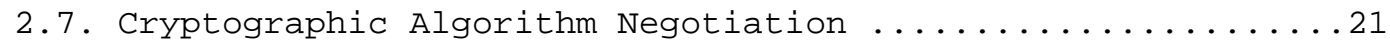

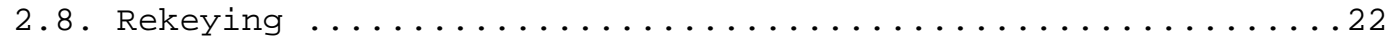

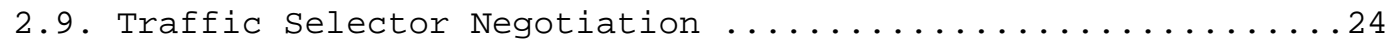

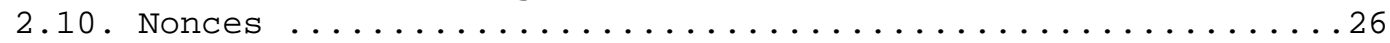

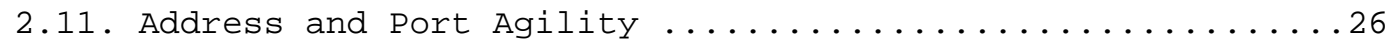

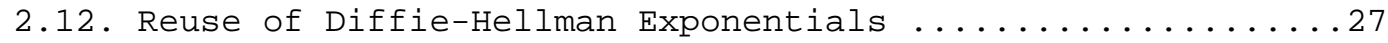

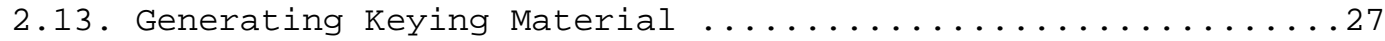

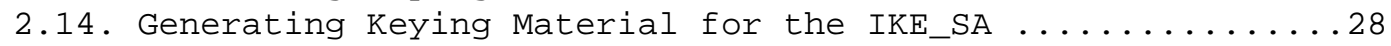

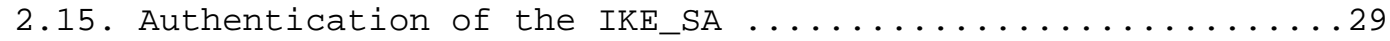

2.16. Extensible Authentication Protocol Methods ............31

2.17. Generating Keying Material for CHILD_SAs ....................33

2.18. Rekeying IKE_SAs Using a CREATE_CHILD_SA exchange .......34

2.19. Requesting an Internal Address on a Remote Network ......34

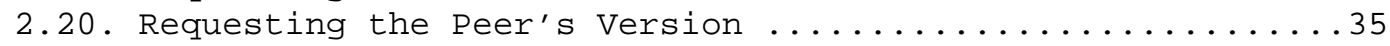

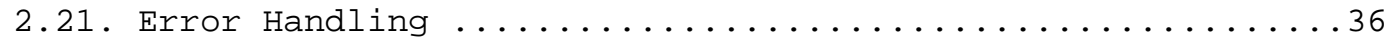

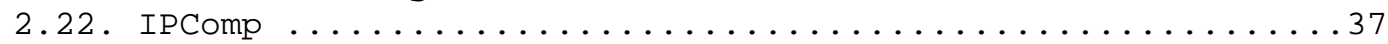

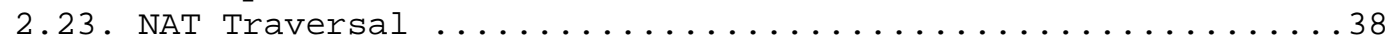

2.24. Explicit Congestion Notification (ECN) ...........40

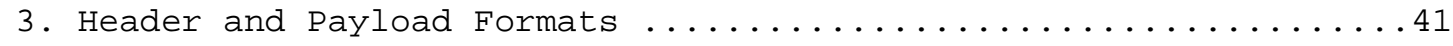

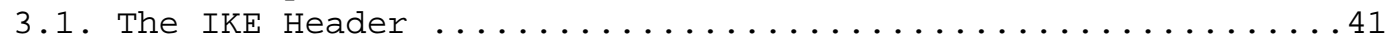

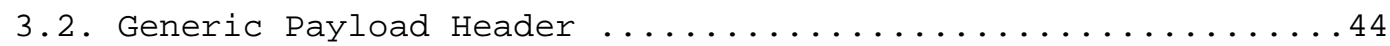

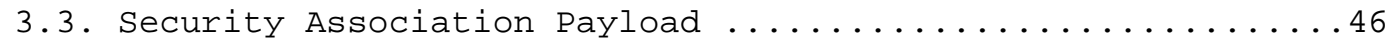

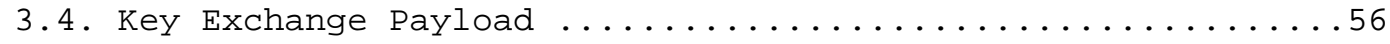

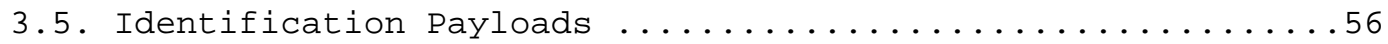

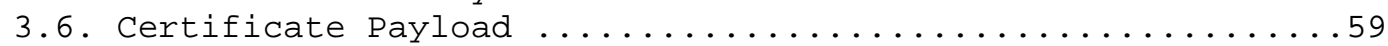

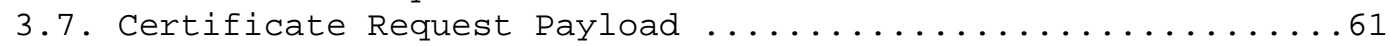

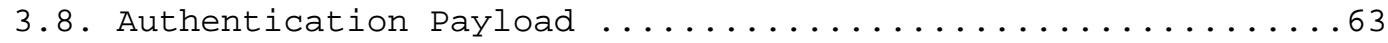

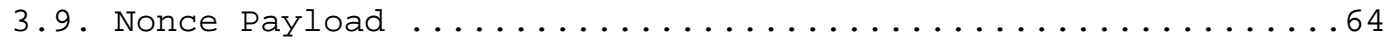

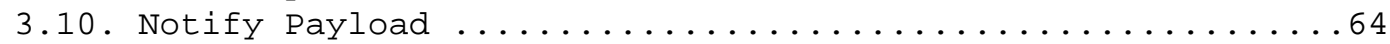

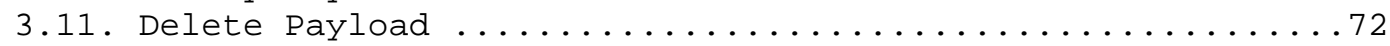

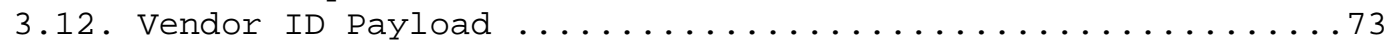

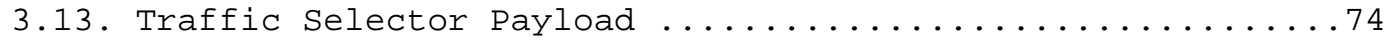

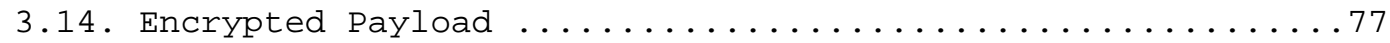




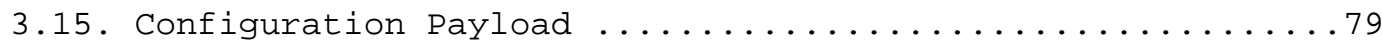

3.16. Extensible Authentication Protocol (EAP) Payload ........84

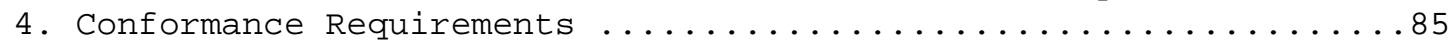

5. Security Considerations . ......................... 88

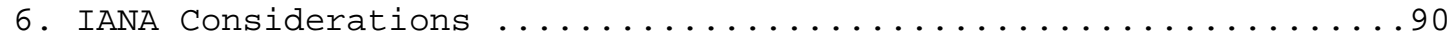

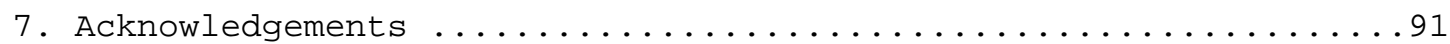

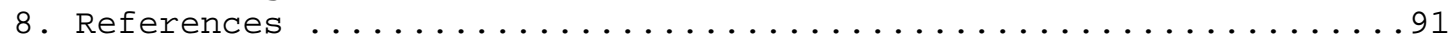

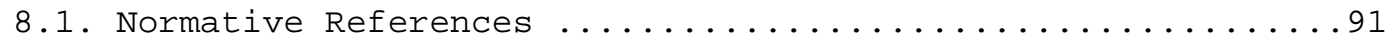

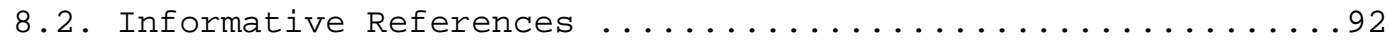

Appendix A: Summary of Changes from IKEv1 ................. 96

Appendix B: Diffie-Hellman Groups ....................... 97

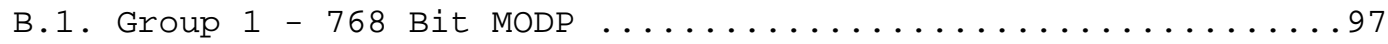

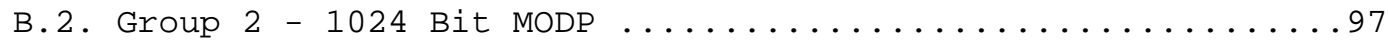

1. Introduction

IP Security (IPsec) provides confidentiality, data integrity, access control, and data source authentication to IP datagrams. These services are provided by maintaining shared state between the source and the sink of an IP datagram. This state defines, among other things, the specific services provided to the datagram, which cryptographic algorithms will be used to provide the services, and the keys used as input to the cryptographic algorithms.

Establishing this shared state in a manual fashion does not scale well. Therefore, a protocol to establish this state dynamically is needed. This memo describes such a protocol -- the Internet Key Exchange (IKE). This is version 2 of IKE. Version 1 of IKE was defined in RFCs 2407, 2408, and 2409 [Pip98, MSST98, HC98]. This single document is intended to replace all three of those RFCs.

Definitions of the primitive terms in this document (such as Security Association or SA) can be found in [RFC4301].

Keywords "MUST", "MUST NOT", "REQUIRED", "SHOULD", "SHOULD NOT" and "MAY" that appear in this document are to be interpreted as described in [Bra97].

The term "Expert Review" is to be interpreted as defined in [RFC2434].

IKE performs mutual authentication between two parties and establishes an IKE security association (SA) that includes shared secret information that can be used to efficiently establish SAs for Encapsulating Security Payload (ESP) [RFC4303] and/or Authentication Header (AH) [RFC4302] and a set of cryptographic algorithms to be used by the SAs to protect the traffic that they carry. In this document, the term "suite" or "cryptographic suite" refers to a 
complete set of algorithms used to protect an SA. An initiator proposes one or more suites by listing supported algorithms that can be combined into suites in a mix-and-match fashion. IKE can also negotiate use of IP Compression (IPComp) [IPCOMP] in connection with an ESP and/or AH SA. We call the IKE SA an "IKE_SA". The SAs for ESP and/or AH that get set up through that IKE_SA we call "CHILD_SAS".

All IKE communications consist of pairs of messages: a request and a response. The pair is called an "exchange". We call the first messages establishing an IKE_SA IKE_SA_INIT and IKE_AUTH exchanges and subsequent IKE exchanges CREATE_CHILD_SA or INFORMATIONAL exchanges. In the common case, there is a single IKE_SA_INIT exchange and a single IKE_AUTH exchange (a total of four messages) to establish the IKE_SA and the first CHILD_SA. In exceptional cases, there may be more than one of each of these exchanges. In all cases, all IKE_SA_INIT exchanges MUST complete before any other exchange type, then all IKE_AUTH exchanges MUST complete, and following that any number of CREATE_CHILD_SA and INFORMATIONAL exchanges may occur in any order. In some scenarios, only a single CHILD_SA is needed between the IPsec endpoints, and therefore there would be no additional exchanges. Subsequent exchanges MAY be used to establish additional CHILD_SAs between the same authenticated pair of endpoints and to perform housekeeping functions.

IKE message flow always consists of a request followed by a response. It is the responsibility of the requester to ensure reliability. If the response is not received within a timeout interval, the requester needs to retransmit the request (or abandon the connection).

The first request/response of an IKE session (IKE_SA_INIT) negotiates security parameters for the IKE_SA, sends nonces, and sends DiffieHellman values.

The second request/response (IKE_AUTH) transmits identities, proves knowledge of the secrets corresponding to the two identities, and sets up an SA for the first (and often only) AH and/or ESP CHILD_SA.

The types of subsequent exchanges are CREATE_CHILD_SA (which creates a CHILD_SA) and INFORMATIONAL (which deletes an SA, reports error conditions, or does other housekeeping). Every request requires a response. An INFORMATIONAL request with no payloads (other than the empty Encrypted payload required by the syntax) is commonly used as a check for liveness. These subsequent exchanges cannot be used until the initial exchanges have completed. 
In the description that follows, we assume that no errors occur. Modifications to the flow should errors occur are described in section 2.21 .

\subsection{Usage Scenarios}

IKE is expected to be used to negotiate ESP and/or AH SAs in a number of different scenarios, each with its own special requirements.

1.1.1. Security Gateway to Security Gateway Tunnel

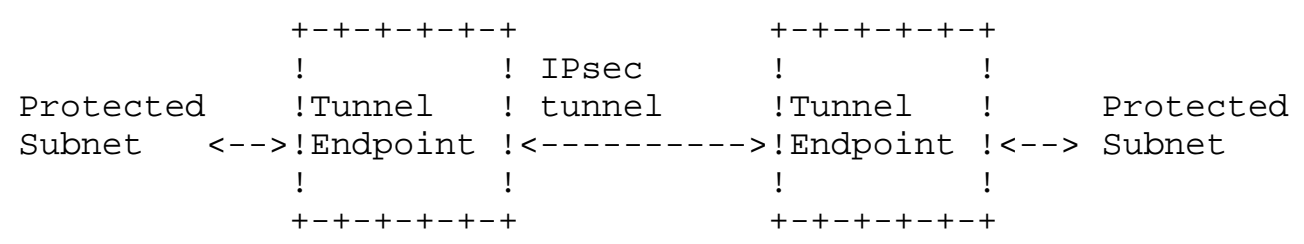

Figure 1: Security Gateway to Security Gateway Tunnel

In this scenario, neither endpoint of the IP connection implements IPsec, but network nodes between them protect traffic for part of the way. Protection is transparent to the endpoints, and depends on ordinary routing to send packets through the tunnel endpoints for processing. Each endpoint would announce the set of addresses "behind" it, and packets would be sent in tunnel mode where the inner IP header would contain the IP addresses of the actual endpoints.

1.1.2. Endpoint-to-Endpoint Transport

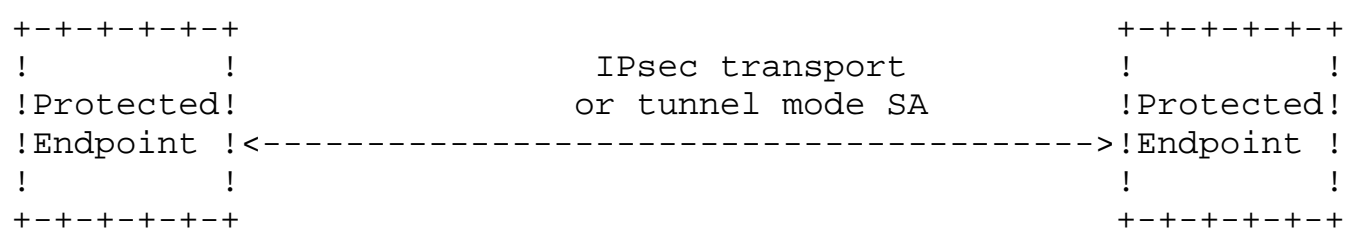

Figure 2: Endpoint to Endpoint

In this scenario, both endpoints of the IP connection implement IPsec, as required of hosts in [RFC4301]. Transport mode will commonly be used with no inner IP header. If there is an inner IP header, the inner addresses will be the same as the outer addresses. A single pair of addresses will be negotiated for packets to be protected by this SA. These endpoints MAY implement application layer access controls based on the IPsec authenticated identities of the participants. This scenario enables the end-to-end security that has been a guiding principle for the Internet since [RFC1958], 
[RFC2775], and a method of limiting the inherent problems with complexity in networks noted by [RFC3439]. Although this scenario may not be fully applicable to the IPv4 Internet, it has been deployed successfully in specific scenarios within intranets using IKEv1. It should be more broadly enabled during the transition to IPv6 and with the adoption of IKEv2.

It is possible in this scenario that one or both of the protected endpoints will be behind a network address translation (NAT) node, in which case the tunneled packets will have to be UDP encapsulated so that port numbers in the UDP headers can be used to identify individual endpoints "behind" the NAT (see section 2.23).

1.1.3. Endpoint to Security Gateway Tunnel

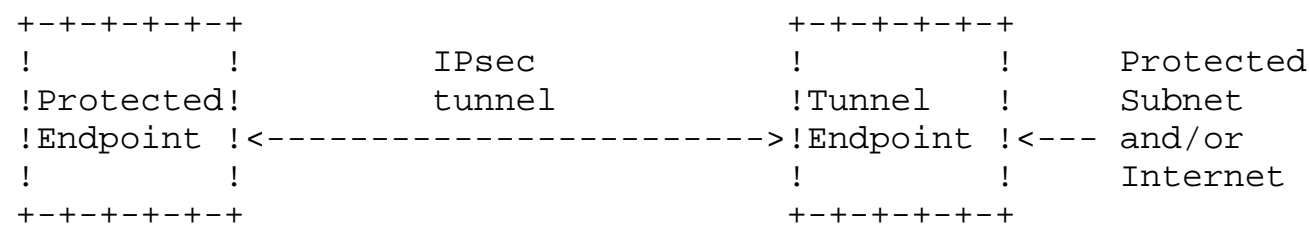

Figure 3: Endpoint to Security Gateway Tunnel

In this scenario, a protected endpoint (typically a portable roaming computer) connects back to its corporate network through an IPsecprotected tunnel. It might use this tunnel only to access information on the corporate network, or it might tunnel all of its traffic back through the corporate network in order to take advantage of protection provided by a corporate firewall against Internet-based attacks. In either case, the protected endpoint will want an IP address associated with the security gateway so that packets returned to it will go to the security gateway and be tunneled back. This IP address may be static or may be dynamically allocated by the security gateway. In support of the latter case, IKEv2 includes a mechanism for the initiator to request an IP address owned by the security gateway for use for the duration of its SA.

In this scenario, packets will use tunnel mode. On each packet from the protected endpoint, the outer IP header will contain the source IP address associated with its current location (i.e., the address that will get traffic routed to the endpoint directly), while the inner IP header will contain the source IP address assigned by the security gateway (i.e., the address that will get traffic routed to the security gateway for forwarding to the endpoint). The outer destination address will always be that of the security gateway, while the inner destination address will be the ultimate destination for the packet. 
In this scenario, it is possible that the protected endpoint will be behind a NAT. In that case, the IP address as seen by the security gateway will not be the same as the IP address sent by the protected endpoint, and packets will have to be UDP encapsulated in order to be routed properly.

\subsubsection{Other Scenarios}

Other scenarios are possible, as are nested combinations of the above. One notable example combines aspects of 1.1 .1 and 1.1.3. A subnet may make all external accesses through a remote security gateway using an IPsec tunnel, where the addresses on the subnet are routed to the security gateway by the rest of the Internet. An example would be someone's home network being virtually on the Internet with static IP addresses even though connectivity is provided by an ISP that assigns a single dynamically assigned IP address to the user's security gateway (where the static IP addresses and an IPsec relay are provided by a third party located elsewhere).

\subsection{The Initial Exchanges}

Communication using IKE always begins with IKE_SA_INIT and IKE_AUTH exchanges (known in IKEv1 as Phase 1). These initial exchanges normally consist of four messages, though in some scenarios that number can grow. All communications using IKE consist of request/response pairs. We'll describe the base exchange first, followed by variations. The first pair of messages (IKE_SA_INIT) negotiate cryptographic algorithms, exchange nonces, and do a Diffie-Hellman exchange [DH].

The second pair of messages (IKE_AUTH) authenticate the previous messages, exchange identities and certificates, and establish the first CHILD_SA. Parts of these messages are encrypted and integrity protected with keys established through the IKE_SA_INIT exchange, so the identities are hidden from eavesdroppers and all fields in all the messages are authenticated.

In the following descriptions, the payloads contained in the message are indicated by names as listed below.

\begin{tabular}{ll} 
Notation & \multicolumn{1}{c}{ Payload } \\
AUTH & Authentication \\
CERT & Certificate \\
CERTREQ & Certificate Request \\
CP & Configuration \\
D & Delete \\
E & Encrypted
\end{tabular}




$\begin{array}{ll}\text { EAP } & \text { Extensible Authentication } \\ \text { HDR } & \text { IKE Header } \\ \text { IDi } & \text { Identification - Initiator } \\ \text { IDr } & \text { Identification - Responder } \\ \text { KE } & \text { Key Exchange } \\ \text { Ni, Nr } & \text { Nonce } \\ \text { N } & \text { Notify } \\ \text { SA } & \text { Security Association } \\ \text { TSi } & \text { Traffic Selector - Initiator } \\ \text { TSr } & \text { Traffic Selector - Responder } \\ \text { V } & \text { Vendor ID }\end{array}$

The details of the contents of each payload are described in section 3. Payloads that may optionally appear will be shown in brackets, such as [CERTREQ], indicate that optionally a certificate request payload can be included.

The initial exchanges are as follows:

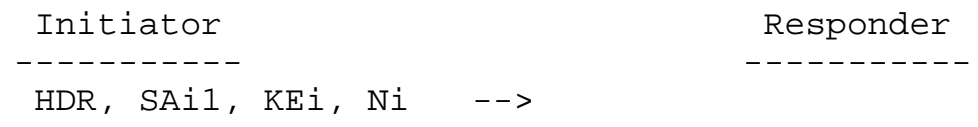

HDR contains the Security Parameter Indexes (SPIs), version numbers, and flags of various sorts. The SAil payload states the cryptographic algorithms the initiator supports for the IKE_SA. The $\mathrm{KE}$ payload sends the initiator's Diffie-Hellman value. Ni is the initiator's nonce.

$<--\quad H D R, \quad S A r 1, \mathrm{KEr}, \mathrm{Nr}$, [CERTREQ]

The responder chooses a cryptographic suite from the initiator's offered choices and expresses that choice in the SArl payload, completes the Diffie-Hellman exchange with the KEr payload, and sends its nonce in the $\mathrm{Nr}$ payload.

At this point in the negotiation, each party can generate SKEYSEED, from which all keys are derived for that IKE_SA. All but the headers of all the messages that follow are encrypted and integrity protected. The keys used for the encryption and integrity protection are derived from SKEYSEED and are known as SK_e (encryption) and SK_a (authentication, a.k.a. integrity protection). A separate SK_e and SK_a is computed for each direction. In addition to the keys SK_e and SK_a derived from the DH value for protection of the IKE_SA, another quantity SK_d is derived and used for derivation of further keying material for CHILD_SAs. The notation SK \{... $\}$ indicates that these payloads are encrypted and integrity protected using that direction's sk_e and SK_a. 


$$
\begin{aligned}
& \text { HDR, SK \{IDi, [CERT, ] [CERTREQ, ] [IDr, ] } \\
& \text { AUTH, SAi2, TSi, TSr }\} \quad->
\end{aligned}
$$

The initiator asserts its identity with the IDi payload, proves knowledge of the secret corresponding to IDi and integrity protects the contents of the first message using the AUTH payload (see section 2.15). It might also send its certificate(s) in CERT payload(s) and a list of its trust anchors in CERTREQ payload(s). If any CERT payloads are included, the first certificate provided MUST contain the public key used to verify the AUTH field. The optional payload IDr enables the initiator to specify which of the responder's identities it wants to talk to. This is useful when the machine on which the responder is running is hosting multiple identities at the same IP address. The initiator begins negotiation of a CHILD_SA using the SAi2 payload. The final fields (starting with SAi2) are described in the description of the CREATE_CHILD_SA exchange.

$$
\begin{aligned}
&<--\quad H D R, \text { SK }\{\text { IDr, [CERT, ] AUTH, } \\
&\text { SAr2, TSi, TSr }\}
\end{aligned}
$$

The responder asserts its identity with the IDr payload, optionally sends one or more certificates (again with the certificate containing the public key used to verify AUTH listed first), authenticates its identity and protects the integrity of the second message with the AUTH payload, and completes negotiation of a CHILD_SA with the additional fields described below in the CREATE_CHILD_SA exchange.

The recipients of messages 3 and 4 MUST verify that all signatures and MACs are computed correctly and that the names in the ID payloads correspond to the keys used to generate the AUTH payload.

\subsection{The CREATE_CHILD_SA Exchange}

This exchange consists of a single request/response pair, and was referred to as a phase 2 exchange in IKEv1. It MAY be initiated by either end of the IKE_SA after the initial exchanges are completed.

All messages following the initial exchange are cryptographically protected using the cryptographic algorithms and keys negotiated in the first two messages of the IKE exchange. These subsequent messages use the syntax of the Encrypted Payload described in section 3.14. All subsequent messages included an Encrypted Payload, even if they are referred to in the text as "empty".

Either endpoint may initiate a CREATE_CHILD_SA exchange, so in this section the term "initiator" refers to the endpoint initiating this exchange. 
A CHILD_SA is created by sending a CREATE_CHILD_SA request. The CREATE_CHILD_SA request MAY optionally contain a KE payload for an additional Diffie-Hellman exchange to enable stronger guarantees of forward secrecy for the CHILD_SA. The keying material for the CHILD_SA is a function of SK_d established during the establishment of the IKE_SA, the nonces exchanged during the CREATE_CHILD_SA exchange, and the Diffie-Hellman value (if KE payloads are included in the CREATE_CHILD_SA exchange).

In the CHILD_SA created as part of the initial exchange, a second KE payload and nonce MUST NOT be sent. The nonces from the initial exchange are used in computing the keys for the CHILD_SA.

The CREATE_CHILD_SA request contains:

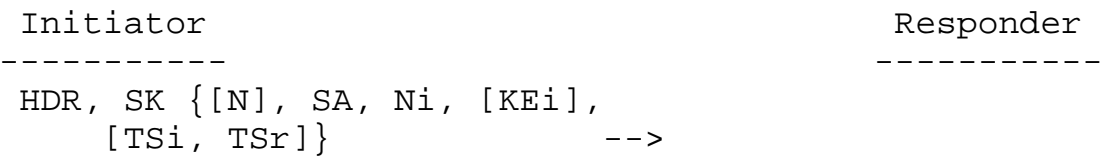

The initiator sends SA offer(s) in the SA payload, a nonce in the Ni payload, optionally a Diffie-Hellman value in the KEi payload, and the proposed traffic selectors in the TSi and TSr payloads. If this CREATE_CHILD_SA exchange is rekeying an existing SA other than the IKE_SA, the leading $N$ payload of type REKEY_SA MUST identify the SA being rekeyed. If this CREATE_CHILD_SA exchange is not rekeying an existing $\mathrm{SA}$, the $\mathrm{N}$ payload MUST be omitted. If the SA offers include different Diffie-Hellman groups, KEi MUST be an element of the group the initiator expects the responder to accept. If it guesses wrong, the CREATE_CHILD_SA exchange will fail, and it will have to retry with a different KEi.

The message following the header is encrypted and the message including the header is integrity protected using the cryptographic algorithms negotiated for the IKE_SA.

The CREATE_CHILD_SA response contains:

$$
\begin{gathered}
<--\quad H D R, \quad S K\{\mathrm{SA}, \mathrm{Nr},[\mathrm{KEr}], \\
[\mathrm{TSi}, \mathrm{TSr}]\}
\end{gathered}
$$

The responder replies (using the same Message ID to respond) with the accepted offer in an SA payload, and a Diffie-Hellman value in the $\mathrm{KEr}$ payload if $\mathrm{KEi}$ was included in the request and the selected cryptographic suite includes that group. If the responder chooses a cryptographic suite with a different group, it MUST reject the request. The initiator SHOULD repeat the request, but now with a $\mathrm{KEi}$ payload from the group the responder selected. 
The traffic selectors for traffic to be sent on that SA are specified in the TS payloads, which may be a subset of what the initiator of the CHILD_SA proposed. Traffic selectors are omitted if this CREATE_CHILD_SA request is being used to change the key of the IKE_SA.

\subsection{The INFORMATIONAL Exchange}

At various points during the operation of an IKE_SA, peers may desire to convey control messages to each other regarding errors or notifications of certain events. To accomplish this, IKE defines an INFORMATIONAL exchange. INFORMATIONAL exchanges MUST ONLY occur after the initial exchanges and are cryptographically protected with the negotiated keys.

Control messages that pertain to an IKE_SA MUST be sent under that IKE_SA. Control messages that pertain to CHILD_SAs MUST be sent under the protection of the IKE_SA which generated them (or its successor if the IKE_SA was replaced for the purpose of rekeying).

Messages in an INFORMATIONAL exchange contain zero or more Notification, Delete, and Configuration payloads. The Recipient of an INFORMATIONAL exchange request MUST send some response (else the Sender will assume the message was lost in the network and will retransmit it). That response MAY be a message with no payloads. The request message in an INFORMATIONAL exchange MAY also contain no payloads. This is the expected way an endpoint can ask the other endpoint to verify that it is alive.

ESP and AH SAs always exist in pairs, with one SA in each direction. When an SA is closed, both members of the pair MUST be closed. When SAs are nested, as when data (and IP headers if in tunnel mode) are encapsulated first with IPComp, then with ESP, and finally with $\mathrm{AH}$ between the same pair of endpoints, all of the SAs MUST be deleted together. Each endpoint MUST close its incoming SAs and allow the other endpoint to close the other SA in each pair. To delete an SA, an INFORMATIONAL exchange with one or more delete payloads is sent listing the SPIs (as they would be expected in the headers of inbound packets) of the SAs to be deleted. The recipient MUST close the designated SAs. Normally, the reply in the INFORMATIONAL exchange will contain delete payloads for the paired SAs going in the other direction. There is one exception. If by chance both ends of a set of SAs independently decide to close them, each may send a delete payload and the two requests may cross in the network. If a node receives a delete request for SAs for which it has already issued a delete request, it MUST delete the outgoing SAs while processing the request and the incoming SAs while processing the response. In that 
case, the responses MUST NOT include delete payloads for the deleted SAs, since that would result in duplicate deletion and could in theory delete the wrong SA.

A node SHOULD regard half-closed connections as anomalous and audit their existence should they persist. Note that this specification nowhere specifies time periods, so it is up to individual endpoints to decide how long to wait. A node MAY refuse to accept incoming data on half-closed connections but MUST NOT unilaterally close them and reuse the SPIs. If connection state becomes sufficiently messed up, a node MAY close the IKE_SA; doing so will implicitly close all SAs negotiated under it. It can then rebuild the SAs it needs on a clean base under a new IKE_SA.

The INFORMATIONAL exchange is defined as:

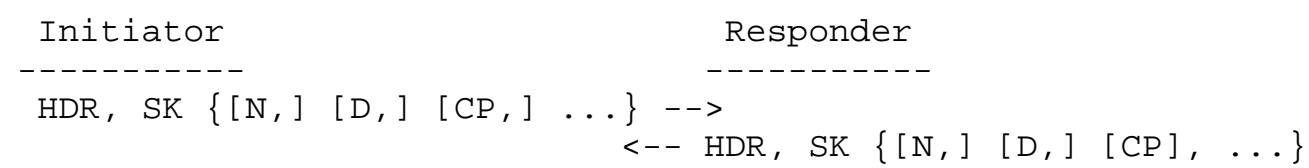

The processing of an INFORMATIONAL exchange is determined by its component payloads.

\subsection{Informational Messages outside of an IKE_SA}

If an encrypted IKE packet arrives on port 500 or 4500 with an unrecognized SPI, it could be because the receiving node has recently crashed and lost state or because of some other system malfunction or attack. If the receiving node has an active IKE_SA to the IP address from whence the packet came, it MAY send a notification of the wayward packet over that IKE_SA in an INFORMATIONAL exchange. If it does not have such an IKE_SA, it MAY send an Informational message without cryptographic protection to the source IP address. Such a message is not part of an informational exchange, and the receiving node MUST NOT respond to it. Doing so could cause a message loop.

\section{IKE Protocol Details and Variations}

IKE normally listens and sends on UDP port 500, though IKE messages may also be received on UDP port 4500 with a slightly different format (see section 2.23). Since UDP is a datagram (unreliable) protocol, IKE includes in its definition recovery from transmission errors, including packet loss, packet replay, and packet forgery. IKE is designed to function so long as (1) at least one of a series of retransmitted packets reaches its destination before timing out; and (2) the channel is not so full of forged and replayed packets so 
as to exhaust the network or CPU capacities of either endpoint. Even in the absence of those minimum performance requirements, IKE is designed to fail cleanly (as though the network were broken).

Although IKEv2 messages are intended to be short, they contain structures with no hard upper bound on size (in particular, X.509 certificates), and IKEv2 itself does not have a mechanism for fragmenting large messages. IP defines a mechanism for fragmentation of oversize UDP messages, but implementations vary in the maximum message size supported. Furthermore, use of IP fragmentation opens an implementation to denial of service attacks [KPS03]. Finally, some NAT and/or firewall implementations may block IP fragments.

All IKEv2 implementations MUST be able to send, receive, and process IKE messages that are up to 1280 bytes long, and they SHOULD be able to send, receive, and process messages that are up to 3000 bytes long. IKEv2 implementations SHOULD be aware of the maximum UDP message size supported and MAY shorten messages by leaving out some certificates or cryptographic suite proposals if that will keep messages below the maximum. Use of the "Hash and URL" formats rather than including certificates in exchanges where possible can avoid most problems. Implementations and configuration should keep in mind, however, that if the URL lookups are possible only after the IPsec SA is established, recursion issues could prevent this technique from working.

\subsection{Use of Retransmission Timers}

All messages in IKE exist in pairs: a request and a response. The setup of an IKE_SA normally consists of two request/response pairs. Once the IKE_SA is set up, either end of the security association may initiate requests at any time, and there can be many requests and responses "in flight" at any given moment. But each message is labeled as either a request or a response, and for each request/response pair one end of the security association is the initiator and the other is the responder.

For every pair of IKE messages, the initiator is responsible for retransmission in the event of a timeout. The responder MUST never retransmit a response unless it receives a retransmission of the request. In that event, the responder MUST ignore the retransmitted request except insofar as it triggers a retransmission of the response. The initiator MUST remember each request until it receives the corresponding response. The responder MUST remember each response until it receives a request whose sequence number is larger than the sequence number in the response plus its window size (see section 2.3). 
IKE is a reliable protocol, in the sense that the initiator MUST retransmit a request until either it receives a corresponding reply OR it deems the IKE security association to have failed and it discards all state associated with the IKE_SA and any CHILD_SAs negotiated using that IKE_SA.

\subsection{Use of Sequence Numbers for Message ID}

Every IKE message contains a Message ID as part of its fixed header. This Message ID is used to match up requests and responses, and to identify retransmissions of messages.

The Message ID is a 32-bit quantity, which is zero for the first IKE request in each direction. The IKE_SA initial setup messages will always be numbered 0 and 1. Each endpoint in the IKE Security Association maintains two "current" Message IDs: the next one to be used for a request it initiates and the next one it expects to see in a request from the other end. These counters increment as requests are generated and received. Responses always contain the same message ID as the corresponding request. That means that after the initial exchange, each integer $n$ may appear as the message ID in four distinct messages: the nth request from the original IKE initiator, the corresponding response, the nth request from the original IKE responder, and the corresponding response. If the two ends make very different numbers of requests, the Message IDs in the two directions can be very different. There is no ambiguity in the messages, however, because the (I) nitiator and (R) esponse bits in the message header specify which of the four messages a particular one is.

Note that Message IDs are cryptographically protected and provide protection against message replays. In the unlikely event that Message IDs grow too large to fit in 32 bits, the IKE_SA MUST be closed. Rekeying an IKE_SA resets the sequence numbers.

\subsection{Window Size for Overlapping Requests}

In order to maximize IKE throughput, an IKE endpoint MAY issue multiple requests before getting a response to any of them if the other endpoint has indicated its ability to handle such requests. For simplicity, an IKE implementation MAY choose to process requests strictly in order and/or wait for a response to one request before issuing another. Certain rules must be followed to ensure interoperability between implementations using different strategies.

After an IKE_SA is set up, either end can initiate one or more requests. These requests may pass one another over the network. An IKE endpoint MUST be prepared to accept and process a request while 
it has a request outstanding in order to avoid a deadlock in this situation. An IKE endpoint SHOULD be prepared to accept and process multiple requests while it has a request outstanding.

An IKE endpoint MUST wait for a response to each of its messages before sending a subsequent message unless it has received a SET_WINDOW_SIZE Notify message from its peer informing it that the peer is prepared to maintain state for multiple outstanding messages in order to allow greater throughput.

An IKE endpoint MUST NOT exceed the peer's stated window size for transmitted IKE requests. In other words, if the responder stated its window size is $\mathrm{N}$, then when the initiator needs to make a request $\mathrm{X}$, it MUST wait until it has received responses to all requests up through request $\mathrm{X}-\mathrm{N}$. An IKE endpoint MUST keep a copy of (or be able to regenerate exactly) each request it has sent until it receives the corresponding response. An IKE endpoint MUST keep a copy of (or be able to regenerate exactly) the number of previous responses equal to its declared window size in case its response was lost and the initiator requests its retransmission by retransmitting the request.

An IKE endpoint supporting a window size greater than one SHOULD be capable of processing incoming requests out of order to maximize performance in the event of network failures or packet reordering.

\subsection{State Synchronization and Connection Timeouts}

An IKE endpoint is allowed to forget all of its state associated with an IKE_SA and the collection of corresponding CHILD_SAs at any time. This is the anticipated behavior in the event of an endpoint crash and restart. It is important when an endpoint either fails or reinitializes its state that the other endpoint detect those conditions and not continue to waste network bandwidth by sending packets over discarded SAs and having them fall into a black hole.

Since IKE is designed to operate in spite of Denial of Service (DoS) attacks from the network, an endpoint MUST NOT conclude that the other endpoint has failed based on any routing information (e.g., ICMP messages) or IKE messages that arrive without cryptographic protection (e.g., Notify messages complaining about unknown SPIs). An endpoint MUST conclude that the other endpoint has failed only when repeated attempts to contact it have gone unanswered for a timeout period or when a cryptographically protected INITIAL_CONTACT notification is received on a different IKE_SA to the same authenticated identity. An endpoint SHOULD suspect that the other endpoint has failed based on routing information and initiate a request to see whether the other endpoint is alive. To check whether the other side is alive, IKE specifies an empty INFORMATIONAL message 
that (like all IKE requests) requires an acknowledgement (note that within the context of an IKE_SA, an "empty" message consists of an IKE header followed by an Encrypted payload that contains no payloads). If a cryptographically protected message has been received from the other side recently, unprotected notifications MAY be ignored. Implementations MUST limit the rate at which they take actions based on unprotected messages.

Numbers of retries and lengths of timeouts are not covered in this specification because they do not affect interoperability. It is suggested that messages be retransmitted at least a dozen times over a period of at least several minutes before giving up on an SA, but different environments may require different rules. To be a good network citizen, retranmission times MUST increase exponentially to avoid flooding the network and making an existing congestion situation worse. If there has only been outgoing traffic on all of the SAs associated with an IKE_SA, it is essential to confirm liveness of the other endpoint to avoid black holes. If no cryptographically protected messages have been received on an IKE_SA or any of its CHILD_SAs recently, the system needs to perform a liveness check in order to prevent sending messages to a dead peer. Receipt of a fresh cryptographically protected message on an IKE_SA or any of its CHILD_SAs ensures liveness of the IKE_SA and all of its CHILD_SAs. Note that this places requirements on the failure modes of an IKE endpoint. An implementation MUST NOT continue sending on any $S A$ if some failure prevents it from receiving on all of the associated SAs. If CHILD_SAs can fail independently from one another without the associated IKE_SA being able to send a delete message, then they MUST be negotiated by separate IKE_SAs.

There is a Denial of Service attack on the initiator of an IKE_SA that can be avoided if the initiator takes the proper care. Since the first two messages of an SA setup are not cryptographically protected, an attacker could respond to the initiator's message before the genuine responder and poison the connection setup attempt. To prevent this, the initiator MAY be willing to accept multiple responses to its first message, treat each as potentially legitimate, respond to it, and then discard all the invalid half-open connections when it receives a valid cryptographically protected response to any one of its requests. Once a cryptographically valid response is received, all subsequent responses should be ignored whether or not they are cryptographically valid.

Note that with these rules, there is no reason to negotiate and agree upon an SA lifetime. If IKE presumes the partner is dead, based on repeated lack of acknowledgement to an IKE message, then the IKE SA and all CHILD_SAs set up through that IKE_SA are deleted. 
An IKE endpoint may at any time delete inactive CHILD_SAs to recover resources used to hold their state. If an IKE endpoint chooses to delete CHILD_SAs, it MUST send Delete payloads to the other end notifying it of the deletion. It MAY similarly time out the IKE_SA. Closing the IKE_SA implicitly closes all associated CHILD_SAs. In this case, an IKE endpoint SHOULD send a Delete payload indicating that it has closed the IKE_SA.

\subsection{Version Numbers and Forward Compatibility}

This document describes version 2.0 of IKE, meaning the major version number is 2 and the minor version number is zero. It is likely that some implementations will want to support both version 1.0 and version 2.0, and in the future, other versions.

The major version number should be incremented only if the packet formats or required actions have changed so dramatically that an older version node would not be able to interoperate with a newer version node if it simply ignored the fields it did not understand and took the actions specified in the older specification. The minor version number indicates new capabilities, and MUST be ignored by a node with a smaller minor version number, but used for informational purposes by the node with the larger minor version number. For example, it might indicate the ability to process a newly defined notification message. The node with the larger minor version number would simply note that its correspondent would not be able to understand that message and therefore would not send it.

If an endpoint receives a message with a higher major version number, it MUST drop the message and SHOULD send an unauthenticated notification message containing the highest version number it supports. If an endpoint supports major version $n$, and major version $\mathrm{m}$, it MUST support all versions between $\mathrm{n}$ and $\mathrm{m}$. If it receives a message with a major version that it supports, it MUST respond with that version number. In order to prevent two nodes from being tricked into corresponding with a lower major version number than the maximum that they both support, IKE has a flag that indicates that the node is capable of speaking a higher major version number.

Thus, the major version number in the IKE header indicates the version number of the message, not the highest version number that the transmitter supports. If the initiator is capable of speaking versions $n, n+1$, and $n+2$, and the responder is capable of speaking versions $n$ and $n+1$, then they will negotiate speaking $n+1$, where the initiator will set the flag indicating its ability to speak a higher version. If they mistakenly (perhaps through an active attacker 
sending error messages) negotiate to version $\mathrm{n}$, then both will notice that the other side can support a higher version number, and they MUST break the connection and reconnect using version $\mathrm{n}+1$.

Note that IKEv1 does not follow these rules, because there is no way in $\mathrm{v} 1$ of noting that you are capable of speaking a higher version number. So an active attacker can trick two v2-capable nodes into speaking v1. When a v2-capable node negotiates down to v1, it SHOULD note that fact in its logs.

Also for forward compatibility, all fields marked RESERVED MUST be set to zero by a version 2.0 implementation and their content MUST be ignored by a version 2.0 implementation ("Be conservative in what you send and liberal in what you receive"). In this way, future versions of the protocol can use those fields in a way that is guaranteed to be ignored by implementations that do not understand them. Similarly, payload types that are not defined are reserved for future use; implementations of version 2.0 MUST skip over those payloads and ignore their contents.

IKEv2 adds a "critical" flag to each payload header for further flexibility for forward compatibility. If the critical flag is set and the payload type is unrecognized, the message MUST be rejected and the response to the IKE request containing that payload MUST include a Notify payload UNSUPPORTED_CRITICAL_PAYLOAD, indicating an unsupported critical payload was included. If the critical flag is not set and the payload type is unsupported, that payload MUST be ignored.

Although new payload types may be added in the future and may appear interleaved with the fields defined in this specification, implementations MUST send the payloads defined in this specification in the order shown in the figures in section 2 and implementations SHOULD reject as invalid a message with those payloads in any other order.

\subsection{Cookies}

The term "cookies" originates with Karn and Simpson [RFC2522] in Photuris, an early proposal for key management with IPsec, and it has persisted. The Internet Security Association and Key Management Protocol (ISAKMP) [MSST98] fixed message header includes two eightoctet fields titled "cookies", and that syntax is used by both IKEv1 and IKEv2 though in IKEv2 they are referred to as the IKE SPI and there is a new separate field in a Notify payload holding the cookie. The initial two eight-octet fields in the header are used as a connection identifier at the beginning of IKE packets. Each endpoint 
chooses one of the two SPIs and SHOULD choose them so as to be unique identifiers of an IKE_SA. An SPI value of zero is special and indicates that the remote SPI value is not yet known by the sender.

Unlike ESP and AH where only the recipient's SPI appears in the header of a message, in IKE the sender's SPI is also sent in every message. Since the SPI chosen by the original initiator of the IKE_SA is always sent first, an endpoint with multiple IKE_SAs open that wants to find the appropriate IKE_SA using the SPI it assigned must look at the I(nitiator) Flag bit in the header to determine whether it assigned the first or the second eight octets.

In the first message of an initial IKE exchange, the initiator will not know the responder's SPI value and will therefore set that field to zero.

An expected attack against IKE is state and CPU exhaustion, where the target is flooded with session initiation requests from forged IP addresses. This attack can be made less effective if an implementation of a responder uses minimal CPU and commits no state to an SA until it knows the initiator can receive packets at the address from which it claims to be sending them. To accomplish this, a responder SHOULD -- when it detects a large number of half-open IKE_SAs -- reject initial IKE messages unless they contain a Notify payload of type COOKIE. It SHOULD instead send an unprotected IKE message as a response and include CoOKIE Notify payload with the cookie data to be returned. Initiators who receive such responses MUST retry the IKE_SA_INIT with a Notify payload of type COOKIE containing the responder supplied cookie data as the first payload and all other payloads unchanged. The initial exchange will then be as follows:

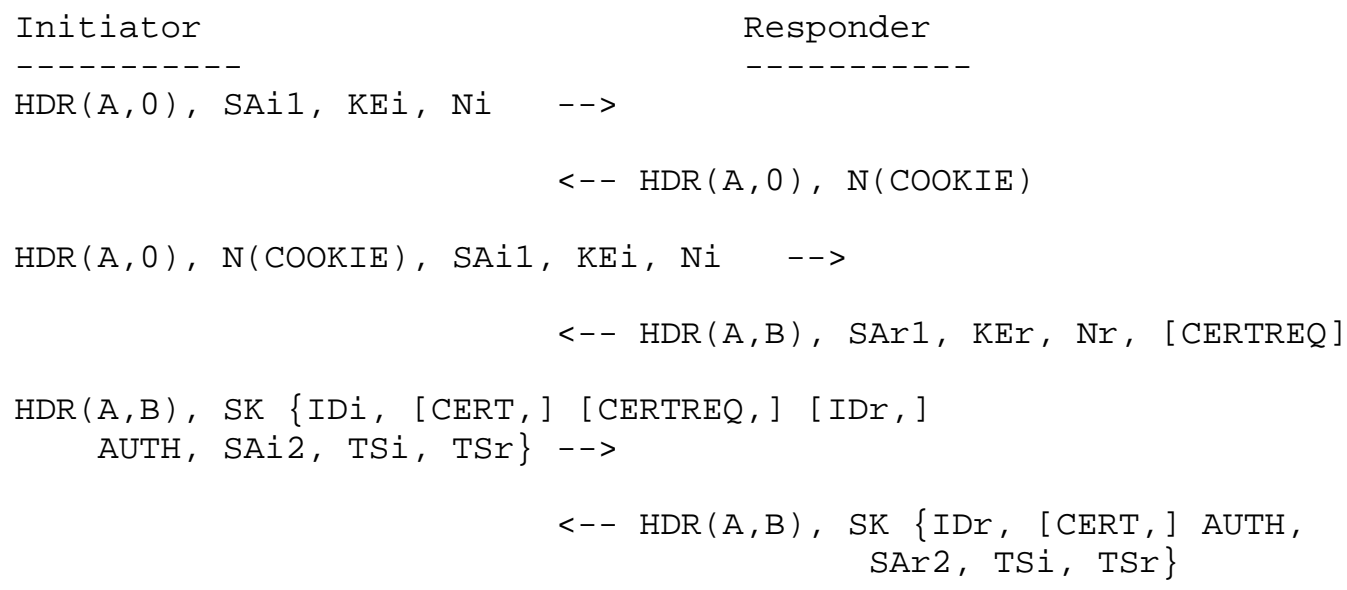


The first two messages do not affect any initiator or responder state except for communicating the cookie. In particular, the message sequence numbers in the first four messages will all be zero and the message sequence numbers in the last two messages will be one. 'A' is the SPI assigned by the initiator, while 'B' is the SPI assigned by the responder.

An IKE implementation SHOULD implement its responder cookie generation in such a way as to not require any saved state to recognize its valid cookie when the second IKE_SA_INIT message arrives. The exact algorithms and syntax they use to generate cookies do not affect interoperability and hence are not specified here. The following is an example of how an endpoint could use cookies to implement limited DOS protection.

A good way to do this is to set the responder cookie to be:

$$
\text { Cookie }=<\text { VersionIDofSecret> } \mid \text { Hash(Ni }|\operatorname{IPi}| \operatorname{SPIi} \mid<\text { secret>) }
$$

where <secret> is a randomly generated secret known only to the responder and periodically changed and | indicates concatenation. <VersionIDofSecret> should be changed whenever <secret> is regenerated. The cookie can be recomputed when the IKE_SA_INIT arrives the second time and compared to the cookie in the received message. If it matches, the responder knows that the cookie was generated since the last change to <secret> and that IPi must be the same as the source address it saw the first time. Incorporating SPIi into the calculation ensures that if multiple IKE_SAs are being set up in parallel they will all get different cookies (assuming the initiator chooses unique SPIi's). Incorporating $\mathrm{Ni}$ into the hash ensures that an attacker who sees only message 2 can't successfully forge a message 3 .

If a new value for <secret> is chosen while there are connections in the process of being initialized, an IKE_SA_INIT might be returned with other than the current <VersionIDofSecret>. The responder in that case MAY reject the message by sending another response with a new cookie or it MAY keep the old value of <secret> around for a short time and accept cookies computed from either one. The responder SHOULD NOT accept cookies indefinitely after <secret> is changed, since that would defeat part of the denial of service protection. The responder SHOULD change the value of <secret> frequently, especially if under attack. 


\subsection{Cryptographic Algorithm Negotiation}

The payload type known as "SA" indicates a proposal for a set of choices of IPsec protocols (IKE, ESP, and/or AH) for the SA as well as cryptographic algorithms associated with each protocol.

An SA payload consists of one or more proposals. Each proposal includes one or more protocols (usually one). Each protocol contains one or more transforms -- each specifying a cryptographic algorithm. Each transform contains zero or more attributes (attributes are needed only if the transform identifier does not completely specify the cryptographic algorithm).

This hierarchical structure was designed to efficiently encode proposals for cryptographic suites when the number of supported suites is large because multiple values are acceptable for multiple transforms. The responder MUST choose a single suite, which MAY be any subset of the SA proposal following the rules below:

Each proposal contains one or more protocols. If a proposal is accepted, the SA response MUST contain the same protocols in the same order as the proposal. The responder MUST accept a single proposal or reject them all and return an error. (Example: if a single proposal contains ESP and AH and that proposal is accepted, both ESP and AH MUST be accepted. If ESP and AH are included in separate proposals, the responder MUST accept only one of them).

Each IPsec protocol proposal contains one or more transforms. Each transform contains a transform type. The accepted cryptographic suite MUST contain exactly one transform of each type included in the proposal. For example: if an ESP proposal includes transforms ENCR_3DES, ENCR_AES w/keysize 128, ENCR_AES w/keysize 256, AUTH_HMAC_MD5, and AUTH_HMAC_SHA, the accepted suite MUST contain one of the ENCR_transforms and one of the AUTH_ transforms. Thus, six combinations are acceptable.

Since the initiator sends its Diffie-Hellman value in the IKE_SA_INIT, it must guess the Diffie-Hellman group that the responder will select from its list of supported groups. If the initiator guesses wrong, the responder will respond with a Notify payload of type INVALID_KE_PAYLOAD indicating the selected group. In this case, the initiator MUST retry the IKE_SA_INIT with the corrected Diffie-Hellman group. The initiator MUST again propose its full set of acceptable cryptographic suites because the rejection message was unauthenticated and otherwise an active attacker could trick the endpoints into negotiating a weaker suite than a stronger one that they both prefer. 


\subsection{Rekeying}

IKE, ESP, and AH security associations use secret keys that SHOULD be used only for a limited amount of time and to protect a limited amount of data. This limits the lifetime of the entire security association. When the lifetime of a security association expires, the security association MUST NOT be used. If there is demand, new security associations MAY be established. Reestablishment of security associations to take the place of ones that expire is referred to as "rekeying".

To allow for minimal IPsec implementations, the ability to rekey SAs without restarting the entire IKE_SA is optional. An implementation MAY refuse all CREATE_CHILD_SA requests within an IKE_SA. If an SA has expired or is about to expire and rekeying attempts using the mechanisms described here fail, an implementation MUST close the IKE_SA and any associated CHILD_SAs and then MAY start new ones. Implementations SHOULD support in-place rekeying of SAs, since doing so offers better performance and is likely to reduce the number of packets lost during the transition.

To rekey a CHILD_SA within an existing IKE_SA, create a new, equivalent SA (see section 2.17 below), and when the new one is established, delete the old one. To rekey an IKE_SA, establish a new equivalent IKE_SA (see section 2.18 below) with the peer to whom the old IKE_SA is shared using a CREATE_CHILD_SA within the existing IKE_SA. An IKE_SA so created inherits all of the original IKE_SA'S CHILD_SAs. Use the new IKE_SA for all control messages needed to maintain the CHILD_SAs created by the old IKE_SA, and delete the old IKE_SA. The Delete payload to delete itself MUST be the last request sent over an IKE_SA.

SAs SHOULD be rekeyed proactively, i.e., the new SA should be established before the old one expires and becomes unusable. Enough time should elapse between the time the new SA is established and the old one becomes unusable so that traffic can be switched over to the new SA.

A difference between IKEV1 and IKEv2 is that in IKEV1 SA lifetimes were negotiated. In IKEv2, each end of the SA is responsible for enforcing its own lifetime policy on the SA and rekeying the SA when necessary. If the two ends have different lifetime policies, the end with the shorter lifetime will end up always being the one to request the rekeying. If an SA bundle has been inactive for a long time and if an endpoint would not initiate the SA in the absence of traffic, the endpoint MAY choose to close the SA instead of rekeying it when its lifetime expires. It SHOULD do so if there has been no traffic since the last time the SA was rekeyed. 
If the two ends have the same lifetime policies, it is possible that both will initiate a rekeying at the same time (which will result in redundant SAs). To reduce the probability of this happening, the timing of rekeying requests SHOULD be jittered (delayed by a random amount of time after the need for rekeying is noticed).

This form of rekeying may temporarily result in multiple similar SAs between the same pairs of nodes. When there are two sAs eligible to receive packets, a node MUST accept incoming packets through either SA. If redundant SAs are created though such a collision, the SA created with the lowest of the four nonces used in the two exchanges SHOULD be closed by the endpoint that created it.

Note that IKEv2 deliberately allows parallel SAs with the same traffic selectors between common endpoints. One of the purposes of this is to support traffic quality of service (QoS) differences among the SAs (see [RFC2474], [RFC2475], and section 4.1 of [RFC2983]). Hence unlike IKEvl, the combination of the endpoints and the traffic selectors may not uniquely identify an SA between those endpoints, so the IKEv1 rekeying heuristic of deleting SAs on the basis of duplicate traffic selectors SHOULD NOT be used.

The node that initiated the surviving rekeyed SA SHOULD delete the replaced SA after the new one is established.

There are timing windows -- particularly in the presence of lost packets -- where endpoints may not agree on the state of an SA. The responder to a CREATE_CHILD_SA MUST be prepared to accept messages on an SA before sending its response to the creation request, so there is no ambiguity for the initiator. The initiator MAY begin sending on an SA as soon as it processes the response. The initiator, however, cannot receive on a newly created SA until it receives and processes the response to its CREATE_CHILD_SA request. How, then, is the responder to know when it is OK to send on the newly created SA?

From a technical correctness and interoperability perspective, the responder MAY begin sending on an SA as soon as it sends its response to the CREATE_CHILD_SA request. In some situations, however, this could result in packets unnecessarily being dropped, so an implementation MAY want to defer such sending.

The responder can be assured that the initiator is prepared to receive messages on an $\mathrm{SA}$ if either (1) it has received a cryptographically valid message on the new SA, or (2) the new SA rekeys an existing $\mathrm{SA}$ and it receives an IKE request to close the replaced SA. When rekeying an SA, the responder SHOULD continue to send messages on the old SA until one of those events occurs. When establishing a new SA, the responder MAY defer sending messages on a 
new SA until either it receives one or a timeout has occurred. If an initiator receives a message on an SA for which it has not received a response to its CREATE_CHILD_SA request, it SHOULD interpret that as a likely packet loss and retransmit the CREATE_CHILD_SA request. An initiator MAY send a dummy message on a newly created SA if it has no messages queued in order to assure the responder that the initiator is ready to receive messages.

\subsection{Traffic Selector Negotiation}

When an IP packet is received by an RFC4301-compliant IPsec subsystem and matches a "protect" selector in its Security Policy Database (SPD), the subsystem MUST protect that packet with IPsec. When no SA exists yet, it is the task of IKE to create it. Maintenance of a system's SPD is outside the scope of IKE (see [PFKEY] for an example protocol), though some implementations might update their SPD in connection with the running of IKE (for an example scenario, see section 1.1 .3$)$.

Traffic Selector (TS) payloads allow endpoints to communicate some of the information from their SPD to their peers. TS payloads specify the selection criteria for packets that will be forwarded over the newly set up SA. This can serve as a consistency check in some scenarios to assure that the SPDs are consistent. In others, it guides the dynamic update of the SPD.

Two TS payloads appear in each of the messages in the exchange that creates a CHILD_SA pair. Each TS payload contains one or more Traffic Selectors. Each Traffic Selector consists of an address range (IPV4 or IPV6), a port range, and an IP protocol ID. In support of the scenario described in section 1.1.3, an initiator may request that the responder assign an IP address and tell the initiator what it is.

IKEv2 allows the responder to choose a subset of the traffic proposed by the initiator. This could happen when the configurations of the two endpoints are being updated but only one end has received the new information. Since the two endpoints may be configured by different people, the incompatibility may persist for an extended period even in the absence of errors. It also allows for intentionally different configurations, as when one end is configured to tunnel all addresses and depends on the other end to have the up-to-date list.

The first of the two TS payloads is known as TSi (Traffic Selectorinitiator). The second is known as TSr (Traffic Selector-responder). TSi specifies the source address of traffic forwarded from (or the destination address of traffic forwarded tol the initiator of the CHILD_SA pair. TSr specifies the destination address of the traffic 
forwarded to (or the source address of the traffic forwarded from) the responder of the CHILD_SA pair. For example, if the original initiator request the creation of a CHILD_SA pair, and wishes to tunnel all traffic from subnet 192.0.1.* on the initiator's side to subnet 192.0.2.* on the responder's side, the initiator would include a single traffic selector in each TS payload. TSi would specify the address range (192.0.1.0 - 192.0.1.255) and TSr would specify the address range (192.0.2.0 - 192.0.2.255). Assuming that proposal was acceptable to the responder, it would send identical TS payloads back. (Note: The IP address range 192.0.2.* has been reserved for use in examples in RFCs and similar documents. This document needed two such ranges, and so also used 192.0.1.*. This should not be confused with any actual address.)

The responder is allowed to narrow the choices by selecting a subset of the traffic, for instance by eliminating or narrowing the range of one or more members of the set of traffic selectors, provided the set does not become the NULL set.

It is possible for the responder's policy to contain multiple smaller ranges, all encompassed by the initiator's traffic selector, and with the responder's policy being that each of those ranges should be sent over a different SA. Continuing the example above, the responder might have a policy of being willing to tunnel those addresses to and from the initiator, but might require that each address pair be on a separately negotiated CHILD_SA. If the initiator generated its request in response to an incoming packet from 192.0.1.43 to 192.0.2.123, there would be no way for the responder to determine which pair of addresses should be included in this tunnel, and it would have to make a guess or reject the request with a status of SINGLE_PAIR_REQUIRED.

To enable the responder to choose the appropriate range in this case, if the initiator has requested the SA due to a data packet, the initiator SHOULD include as the first traffic selector in each of TSi and TSr a very specific traffic selector including the addresses in the packet triggering the request. In the example, the initiator would include in TSi two traffic selectors: the first containing the address range (192.0.1.43 - 192.0.1.43) and the source port and IP protocol from the packet and the second containing (192.0.1.0 192.0.1.255) with all ports and IP protocols. The initiator would similarly include two traffic selectors in TSr.

If the responder's policy does not allow it to accept the entire set of traffic selectors in the initiator's request, but does allow him to accept the first selector of TSi and TSr, then the responder MUST narrow the traffic selectors to a subset that includes the 
initiator's first choices. In this example, the responder might respond with TSi being (192.0.1.43 - 192.0.1.43) with all ports and IP protocols.

If the initiator creates the CHILD_SA pair not in response to an arriving packet, but rather, say, upon startup, then there may be no specific addresses the initiator prefers for the initial tunnel over any other. In that case, the first values in TSi and TSr MAY be ranges rather than specific values, and the responder chooses a subset of the initiator's TSi and TSr that are acceptable. If more than one subset is acceptable but their union is not, the responder MUST accept some subset and MAY include a Notify payload of type ADDITIONAL_TS_POSSIBLE to indicate that the initiator might want to try again. This case will occur only when the initiator and responder are configured differently from one another. If the initiator and responder agree on the granularity of tunnels, the initiator will never request a tunnel wider than the responder will accept. Such misconfigurations SHOULD be recorded in error logs.

2.10. Nonces

The IKE_SA_INIT messages each contain a nonce. These nonces are used as inputs to cryptographic functions. The CREATE_CHILD_SA request and the CREATE_CHILD_SA response also contain nonces. These nonces are used to add freshness to the key derivation technique used to obtain keys for CHILD_SA, and to ensure creation of strong pseudorandom bits from the Diffie-Hellman key. Nonces used in IKEv2 MUST be randomly chosen, MUST be at least 128 bits in size, and MUST be at least half the key size of the negotiated prf. ("prf" refers to "pseudo-random function", one of the cryptographic algorithms negotiated in the IKE exchange.) If the same random number source is used for both keys and nonces, care must be taken to ensure that the latter use does not compromise the former.

\subsection{Address and Port Agility}

IKE runs over UDP ports 500 and 4500, and implicitly sets up ESP and $\mathrm{AH}$ associations for the same IP addresses it runs over. The IP addresses and ports in the outer header are, however, not themselves cryptographically protected, and IKE is designed to work even through Network Address Translation (NAT) boxes. An implementation MUST accept incoming requests even if the source port is not 500 or 4500, and MUST respond to the address and port from which the request was received. It MUST specify the address and port at which the request was received as the source address and port in the response. IKE functions identically over IPv4 or IPv6. 


\subsection{Reuse of Diffie-Hellman Exponentials}

IKE generates keying material using an ephemeral Diffie-Hellman exchange in order to gain the property of "perfect forward secrecy". This means that once a connection is closed and its corresponding keys are forgotten, even someone who has recorded all of the data from the connection and gets access to all of the long-term keys of the two endpoints cannot reconstruct the keys used to protect the conversation without doing a brute force search of the session key space.

Achieving perfect forward secrecy requires that when a connection is closed, each endpoint MUST forget not only the keys used by the connection but also any information that could be used to recompute those keys. In particular, it MUST forget the secrets used in the Diffie-Hellman calculation and any state that may persist in the state of a pseudo-random number generator that could be used to recompute the Diffie-Hellman secrets.

Since the computing of Diffie-Hellman exponentials is computationally expensive, an endpoint may find it advantageous to reuse those exponentials for multiple connection setups. There are several reasonable strategies for doing this. An endpoint could choose a new exponential only periodically though this could result in less-thanperfect forward secrecy if some connection lasts for less than the lifetime of the exponential. Or it could keep track of which exponential was used for each connection and delete the information associated with the exponential only when some corresponding connection was closed. This would allow the exponential to be reused without losing perfect forward secrecy at the cost of maintaining more state.

Decisions as to whether and when to reuse Diffie-Hellman exponentials is a private decision in the sense that it will not affect interoperability. An implementation that reuses exponentials MAY choose to remember the exponential used by the other endpoint on past exchanges and if one is reused to avoid the second half of the calculation.

\subsection{Generating Keying Material}

In the context of the IKE_SA, four cryptographic algorithms are negotiated: an encryption algorithm, an integrity protection algorithm, a Diffie-Hellman group, and a pseudo-random function (prf). The pseudo-random function is used for the construction of keying material for all of the cryptographic algorithms used in both the IKE_SA and the CHILD_SAs. 
We assume that each encryption algorithm and integrity protection algorithm uses a fixed-size key and that any randomly chosen value of that fixed size can serve as an appropriate key. For algorithms that accept a variable length key, a fixed key size MUST be specified as part of the cryptographic transform negotiated. For algorithms for which not all values are valid keys (such as DES or 3DES with key parity), the algorithm by which keys are derived from arbitrary values MUST be specified by the cryptographic transform. For integrity protection functions based on Hashed Message Authentication Code (HMAC), the fixed key size is the size of the output of the underlying hash function. When the prf function takes a variable length key, variable length data, and produces a fixed-length output (e.g., when using HMAC), the formulas in this document apply. When the key for the prf function has fixed length, the data provided as a key is truncated or padded with zeros as necessary unless exceptional processing is explained following the formula.

Keying material will always be derived as the output of the negotiated prf algorithm. Since the amount of keying material needed may be greater than the size of the output of the prf algorithm, we will use the prf iteratively. We will use the terminology prft to describe the function that outputs a pseudo-random stream based on the inputs to a prf as follows: (where | indicates concatenation)

$\operatorname{prft}(\mathrm{K}, \mathrm{S})=\mathrm{T} 1|\mathrm{~T} 2| \mathrm{T} 3|\mathrm{~T} 4| \ldots$

where:

$\mathrm{T} 1=\operatorname{prf}(\mathrm{K}, \mathrm{S} \mid 0 \times 01)$

$\mathrm{T} 2=\operatorname{prf}(\mathrm{K}, \mathrm{T} 1|\mathrm{~S}| \mathrm{0} 02)$

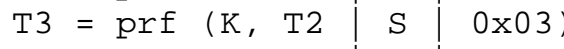

$\mathrm{T} 4=\operatorname{prf}(\mathrm{K}, \mathrm{T} 3|\mathrm{~S}| 0 \times 04)$

continuing as needed to compute all required keys. The keys are taken from the output string without regard to boundaries (e.g., if the required keys are a 256-bit Advanced Encryption Standard (AES) key and a 160-bit HMAC key, and the prf function generates 160 bits, the AES key will come from T1 and the beginning of $\mathrm{T} 2$, while the HMAC key will come from the rest of T2 and the beginning of T3) .

The constant concatenated to the end of each string feeding the prf is a single octet. prft in this document is not defined beyond 255 times the size of the prf output.

\subsection{Generating Keying Material for the IKE_SA}

The shared keys are computed as follows. A quantity called SKEYSEED is calculated from the nonces exchanged during the IKE_SA_INIT exchange and the Diffie-Hellman shared secret established during that 
exchange. SKEYSEED is used to calculate seven other secrets: SK_d used for deriving new keys for the CHILD_SAs established with this IKE_SA; SK_ai and SK_ar used as a key to the integrity protection algorithm for authenticating the component messages of subsequent exchanges; Sk_ei and Sk_er used for encrypting (and of course decrypting) all subsequent exchanges; and SK_pi and SK_pr, which are used when generating an AUTH payload.

SKEYSEED and its derivatives are computed as follows:

$$
\begin{aligned}
& \text { SKEYSEED }=\operatorname{prf}\left(\mathrm{Ni} \mid \mathrm{Nr}, \mathrm{g}^{\wedge} \mathrm{ir}\right) \\
& \begin{array}{r}
\text { SK_d } \mid \text { SK_ai } \mid \text { SK_ar } \mid \text { SK_ei } \mid \text { SK_er } \mid \text { SK_pi } \mid \text { SK_pr }\}=\text { prf+ } \\
\text { (SKEYSEED, Ni }|\operatorname{Nr}| \text { SPIi } \mid \text { SPIr) }
\end{array}
\end{aligned}
$$

(indicating that the quantities SK_d, SK_ai, SK_ar, SK_ei, Sk_er, SK_pi, and SK_pr are taken in order from the generated bits of the prft). $g^{\wedge} i r$ is the shared secret from the ephemeral Diffie-Hellman exchange. $g^{\wedge} i r$ is represented as a string of octets in big endian order padded with zeros if necessary to make it the length of the modulus. Ni and $\mathrm{Nr}$ are the nonces, stripped of any headers. If the negotiated prf takes a fixed-length key and the lengths of $\mathrm{Ni}$ and $\mathrm{Nr}$ do not add up to that length, half the bits must come from $\mathrm{Ni}$ and half from Nr, taking the first bits of each.

The two directions of traffic flow use different keys. The keys used to protect messages from the original initiator are SK_ai and SK_ei. The keys used to protect messages in the other direction are Sk_ar and Sk_er. Each algorithm takes a fixed number of bits of keying material, which is specified as part of the algorithm. For integrity algorithms based on a keyed hash, the key size is always equal to the length of the output of the underlying hash function.

\subsection{Authentication of the IKE_SA}

When not using extensible authentication (see section 2.16), the peers are authenticated by having each sign (or MAC using a shared secret as the key) a block of data. For the responder, the octets to be signed start with the first octet of the first SPI in the header of the second message and end with the last octet of the last payload in the second message. Appended to this (for purposes of computing the signature) are the initiator's nonce $\mathrm{Ni}$ (just the value, not the payload containing it), and the value prf(SK_pr, IDr') where IDr' is the responder's ID payload excluding the fixed header. Note that neither the nonce $\mathrm{Ni}$ nor the value prf(SK_pr, IDr') are transmitted. Similarly, the initiator signs the first message, starting with the first octet of the first SPI in the header and ending with the last octet of the last payload. Appended to this (for purposes of 
computing the signature) are the responder's nonce $\mathrm{Nr}$, and the value prf(SK_pi, IDi'). In the above calculation, IDi' and IDr' are the entire ID payloads excluding the fixed header. It is critical to the security of the exchange that each side sign the other side's nonce.

Note that all of the payloads are included under the signature, including any payload types not defined in this document. If the first message of the exchange is sent twice (the second time with a responder cookie and/or a different Diffie-Hellman group), it is the second version of the message that is signed.

Optionally, messages 3 and 4 MAY include a certificate, or certificate chain providing evidence that the key used to compute a digital signature belongs to the name in the ID payload. The signature or MAC will be computed using algorithms dictated by the type of key used by the signer, and specified by the Auth Method field in the Authentication payload. There is no requirement that the initiator and responder sign with the same cryptographic algorithms. The choice of cryptographic algorithms depends on the type of key each has. In particular, the initiator may be using a shared key while the responder may have a public signature key and certificate. It will commonly be the case (but it is not required) that if a shared secret is used for authentication that the same key is used in both directions. Note that it is a common but typically insecure practice to have a shared key derived solely from a userchosen password without incorporating another source of randomness.

This is typically insecure because user-chosen passwords are unlikely to have sufficient unpredictability to resist dictionary attacks and these attacks are not prevented in this authentication method. (Applications using password-based authentication for bootstrapping and IKE_SA should use the authentication method in section 2.16, which is designed to prevent off-line dictionary attacks.) The preshared key SHOULD contain as much unpredictability as the strongest key being negotiated. In the case of a pre-shared key, the AUTH value is computed as:

$$
\text { AUTH }=\text { prf(prf(Shared Secret, "Key Pad for IKEv2"), <msg octets>) }
$$

where the string "Key Pad for IKEv2" is 17 ASCII characters without null termination. The shared secret can be variable length. The pad string is added so that if the shared secret is derived from a password, the IKE implementation need not store the password in cleartext, but rather can store the value prf(Shared Secret, "Key Pad for IKEv2"), which could not be used as a password equivalent for protocols other than IKEv2. As noted above, deriving the shared secret from a password is not secure. This construction is used because it is anticipated that people will do it anyway. The 
management interface by which the shared secret is provided MUST accept ASCII strings of at least 64 octets and MUST NOT add a null terminator before using them as shared secrets. It MUST also accept a HEX encoding of the shared secret. The management interface MAY accept other encodings if the algorithm for translating the encoding to a binary string is specified. If the negotiated prf takes a fixed-size key, the shared secret MUST be of that fixed size.

\subsection{Extensible Authentication Protocol Methods}

In addition to authentication using public key signatures and shared secrets, IKE supports authentication using methods defined in RFC 3748 [EAP]. Typically, these methods are asymmetric (designed for a user authenticating to a server), and they may not be mutual. For this reason, these protocols are typically used to authenticate the initiator to the responder and MUST be used in conjunction with a public key signature based authentication of the responder to the initiator. These methods are often associated with mechanisms referred to as "Legacy Authentication" mechanisms.

While this memo references [EAP] with the intent that new methods can be added in the future without updating this specification, some simpler variations are documented here and in section 3.16. [EAP] defines an authentication protocol requiring a variable number of messages. Extensible Authentication is implemented in IKE as additional IKE_AUTH exchanges that MUST be completed in order to initialize the IKE_SA.

An initiator indicates a desire to use extensible authentication by leaving out the AUTH payload from message 3. By including an IDi payload but not an AUTH payload, the initiator has declared an identity but has not proven it. If the responder is willing to use an extensible authentication method, it will place an Extensible Authentication Protocol (EAP) payload in message 4 and defer sending SAr2, TSi, and TSr until initiator authentication is complete in a subsequent IKE_AUTH exchange. In the case of a minimal extensible authentication, the initial SA establishment will appear as follows: 


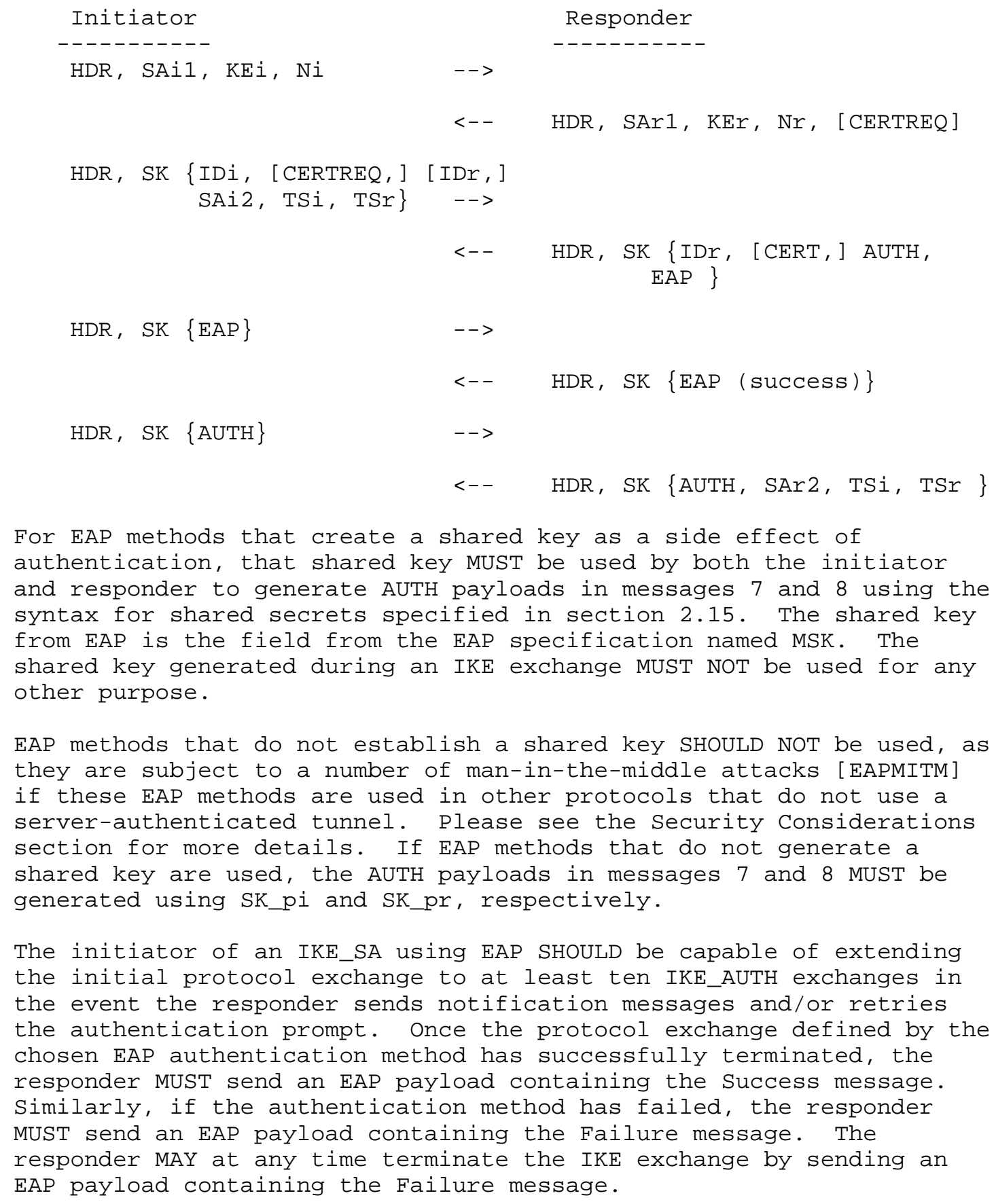


Following such an extended exchange, the EAP AUTH payloads MUST be included in the two messages following the one containing the EAP Success message.

\subsection{Generating Keying Material for CHILD_SAs}

A single CHILD_SA is created by the IKE_AUTH exchange, and additional CHILD_SAs can optionally be created in CREATE_CHILD_SA exchanges. Keying material for them is generated as follows:

$$
\text { KEYMAT }=\operatorname{prf}+\left(\mathrm{SK} \_\mathrm{d}, \mathrm{Ni} \mid \mathrm{Nr}\right)
$$

Where $\mathrm{Ni}$ and $\mathrm{Nr}$ are the nonces from the IKE_SA_INIT exchange if this request is the first CHILD_SA created or the fresh $\mathrm{Ni}$ and $\mathrm{Nr}$ from the CREATE_CHILD_SA exchange if this is a subsequent creation.

For CREATE_CHILD_SA exchanges including an optional Diffie-Hellman exchange, the keying material is defined as:

$$
\text { KEYMAT } \left.=\text { prf+(SK_d, } g^{\wedge} \text { ir (new) }|\mathrm{Ni}| \mathrm{Nr}\right)
$$

where $g^{\wedge}$ ir (new) is the shared secret from the ephemeral DiffieHellman exchange of this CREATE_CHILD_SA exchange (represented as an octet string in big endian order padded with zeros in the high-order bits if necessary to make it the length of the modulus).

A single CHILD_SA negotiation may result in multiple security associations. ESP and AH SAs exist in pairs (one in each direction), and four SAs could be created in a single CHILD_SA negotiation if a combination of ESP and $\mathrm{AH}$ is being negotiated.

Keying material MUST be taken from the expanded KEYMAT in the following order:

All keys for SAs carrying data from the initiator to the responder are taken before SAs going in the reverse direction.

If multiple IPsec protocols are negotiated, keying material is taken in the order in which the protocol headers will appear in the encapsulated packet.

If a single protocol has both encryption and authentication keys, the encryption key is taken from the first octets of KEYMAT and the authentication key is taken from the next octets.

Each cryptographic algorithm takes a fixed number of bits of keying material specified as part of the algorithm. 
2.18. Rekeying IKE_SAs Using a CREATE_CHILD_SA exchange

The CREATE_CHILD_SA exchange can be used to rekey an existing IKE_SA (see section 2.8). New initiator and responder SPIs are supplied in the SPI fields. The TS payloads are omitted when rekeying an IKE_SA. SKEYSEED for the new IKE_SA is computed using SK_d from the existing IKE_SA as follows:

$$
\text { SKEYSEED }=\operatorname{prf}\left(\mathrm{SK} \_d(\mathrm{old}),\left[\mathrm{g}^{\wedge} \text { ir (new) }\right]|\mathrm{Ni}| \mathrm{Nr}\right)
$$

where $g^{\wedge}$ ir (new) is the shared secret from the ephemeral DiffieHellman exchange of this CREATE_CHILD_SA exchange (represented as an octet string in big endian order padded with zeros if necessary to make it the length of the modulus) and $\mathrm{Ni}$ and $\mathrm{Nr}$ are the two nonces stripped of any headers.

The new IKE_SA MUST reset its message counters to 0 .

SK_d, SK_ai, SK_ar, SK_ei, and sk_er are computed from SKEYSEED as specified in section 2.14 .

\subsection{Requesting an Internal Address on a Remote Network}

Most commonly occurring in the endpoint-to-security-gateway scenario, an endpoint may need an IP address in the network protected by the security gateway and may need to have that address dynamically assigned. A request for such a temporary address can be included in any request to create a CHILD_SA (including the implicit request in message 3) by including a CP payload.

This function provides address allocation to an IPsec Remote Access Client (IRAC) trying to tunnel into a network protected by an IPsec Remote Access Server (IRAS). Since the IKE_AUTH exchange creates an IKE_SA and a CHILD_SA, the IRAC MUST request the IRAS-controlled address (and optionally other information concerning the protected network) in the IKE_AUTH exchange. The IRAS may procure an address for the IRAC from any number of sources such as a DHCP/BOOTP server or its own address pool.

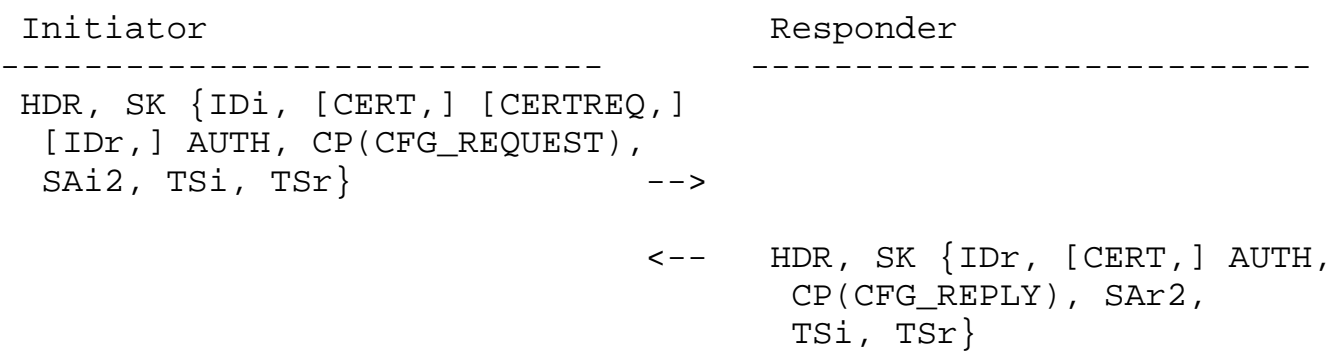


In all cases, the CP payload MUST be inserted before the SA payload. In variations of the protocol where there are multiple IKE_AUTH exchanges, the CP payloads MUST be inserted in the messages containing the SA payloads.

CP (CFG_REQUEST) MUST contain at least an INTERNAL_ADDRESS attribute (either IPV4 or IPV6) but MAY contain any number of additional attributes the initiator wants returned in the response.

For example, message from initiator to responder:

CP (CFG_REQUEST) $=$

INTERNAL_ADDRESS $(0.0 .0 .0)$

INTERNAL_NETMASK $(0.0 .0 .0)$

INTERNAL_DNS $(0.0 .0 .0)$

$\mathrm{TSi}=(0, \overline{0}-65535,0.0 .0 .0-255.255 .255 .255)$

$\mathrm{TSr}=(0,0-65535,0.0 .0 .0-255.255 .255 .255)$

NOTE: Traffic Selectors contain (protocol, port range, address range).

Message from responder to initiator:

CP (CFG_REPLY) $=$ INTERNAL_ADDRESS (192.0.2.202)

INTERNAL_NETMASK (255.255.255.0)

INTERNAL_SUBNET(192.0.2.0/255.255.255.0)

$\mathrm{TSi}=(0, \overline{0}-65535,192.0 .2 .202-192.0 .2 .202)$

$\operatorname{TSr}=(0,0-65535,192.0 .2 .0-192.0 .2 .255)$

All returned values will be implementation dependent. As can be seen in the above example, the IRAS MAY also send other attributes that were not included in CP (CFG_REQUEST) and MAY ignore the non-mandatory attributes that it does not support.

The responder MUST NOT send a CFG_REPLY without having first received a CP (CFG_REQUEST) from the initiator, because we do not want the IRAS to perform an unnecessary configuration lookup if the IRAC cannot process the REPLY. In the case where the IRAS's configuration requires that $\mathrm{CP}$ be used for a given identity IDi, but IRAC has failed to send a CP (CFG_REQUEST), IRAS MUST fail the request, and terminate the IKE exchange with a FAILED_CP_REQUIRED error.

\subsection{Requesting the Peer's Version}

An IKE peer wishing to inquire about the other peer's IKE software version information MAY use the method below. This is an example of a configuration request within an INFORMATIONAL exchange, after the IKE_SA and first CHILD_SA have been created. 
An IKE implementation MAY decline to give out version information prior to authentication or even after authentication to prevent trolling in case some implementation is known to have some security weakness. In that case, it MUST either return an empty string or no $\mathrm{CP}$ payload if $\mathrm{CP}$ is not supported.

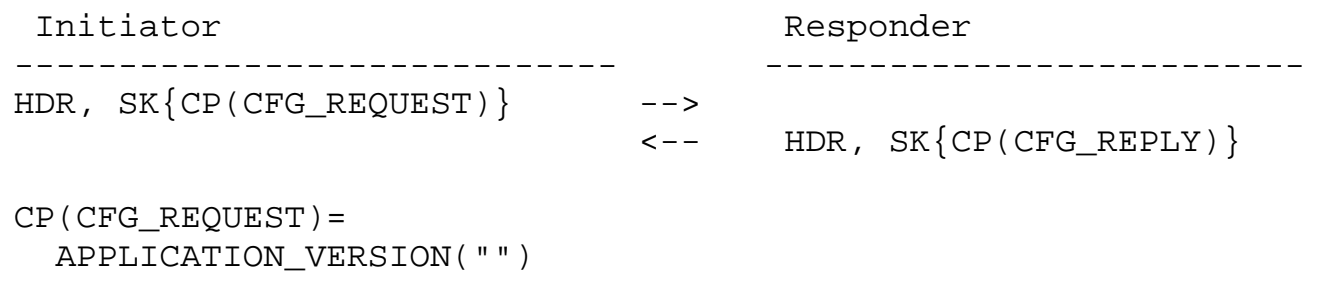

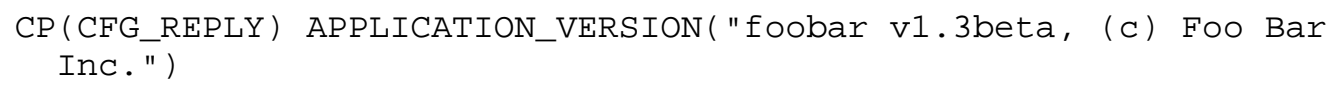

\subsection{Error Handling}

There are many kinds of errors that can occur during IKE processing. If a request is received that is badly formatted or unacceptable for reasons of policy (e.g., no matching cryptographic algorithms), the response MUST contain a Notify payload indicating the error. If an error occurs outside the context of an IKE request (e.g., the node is getting ESP messages on a nonexistent SPI), the node SHOULD initiate an INFORMATIONAL exchange with a Notify payload describing the problem.

Errors that occur before a cryptographically protected IKE_SA is established must be handled very carefully. There is a trade-off between wanting to be helpful in diagnosing a problem and responding to it and wanting to avoid being a dupe in a denial of service attack based on forged messages.

If a node receives a message on UDP port 500 or 4500 outside the context of an IKE_SA known to it (and not a request to start one), it may be the result of a recent crash of the node. If the message is marked as a response, the node MAY audit the suspicious event but MUST NOT respond. If the message is marked as a request, the node MAY audit the suspicious event and MAY send a response. If a response is sent, the response MUST be sent to the IP address and port from whence it came with the same IKE SPIs and the Message ID copied. The response MUST NOT be cryptographically protected and MUST contain a Notify payload indicating INVALID_IKE_SPI.

A node receiving such an unprotected Notify payload MUST NOT respond and MUST NOT change the state of any existing SAs. The message might be a forgery or might be a response the genuine correspondent was 
tricked into sending. A node SHould treat such a message (and also a network message like ICMP destination unreachable) as a hint that there might be problems with SAs to that IP address and SHOULD initiate a liveness test for any such IKE_SA. An implementation SHOULD limit the frequency of such tests to avoid being tricked into participating in a denial of service attack.

A node receiving a suspicious message from an IP address with which it has an IKE_SA MAY send an IKE Notify payload in an IKE

INFORMATIONAL exchange over that SA. The recipient MUST NOT change the state of any $\mathrm{SA}^{\prime} \mathrm{S}$ as a result but SHOULD audit the event to aid in diagnosing malfunctions. A node MUST limit the rate at which it will send messages in response to unprotected messages.

\subsection{IPComp}

Use of IP compression [IPCOMP] can be negotiated as part of the setup of a CHILD_SA. While IP compression involves an extra header in each packet and a compression parameter index (CPI), the virtual "compression association" has no life outside the ESP or AH SA that contains it. Compression associations disappear when the corresponding ESP or AH SA goes away. It is not explicitly mentioned in any DELETE payload.

Negotiation of IP compression is separate from the negotiation of cryptographic parameters associated with a CHILD_SA. A node requesting a CHILD_SA MAY advertise its support for one or more compression algorithms through one or more Notify payloads of type IPCOMP_SUPPORTED. The response MAY indicate acceptance of a single compression algorithm with a Notify payload of type IPCOMP_SUPPORTED. These payloads MUST NOT occur in messages that do not contain SA payloads.

Although there has been discussion of allowing multiple compression algorithms to be accepted and to have different compression algorithms available for the two directions of a CHILD_SA, implementations of this specification MUST NOT accept an IPComp algorithm that was not proposed, MUST NOT accept more than one, and MUST NOT compress using an algorithm other than one proposed and accepted in the setup of the CHILD_SA.

A side effect of separating the negotiation of IPComp from cryptographic parameters is that it is not possible to propose multiple cryptographic suites and propose IP compression with some of them but not others. 


\subsection{NAT Traversal}

Network Address Translation (NAT) gateways are a controversial subject. This section briefly describes what they are and how they are likely to act on IKE traffic. Many people believe that NATs are evil and that we should not design our protocols so as to make them work better. IKEv2 does specify some unintuitive processing rules in order that NATs are more likely to work.

NATs exist primarily because of the shortage of IPv4 addresses, though there are other rationales. IP nodes that are "behind" a NAT have IP addresses that are not globally unique, but rather are assigned from some space that is unique within the network behind the NAT but that are likely to be reused by nodes behind other NATs. Generally, nodes behind NATs can communicate with other nodes behind the same NAT and with nodes with globally unique addresses, but not with nodes behind other NATs. There are exceptions to that rule. When those nodes make connections to nodes on the real Internet, the NAT gateway "translates" the IP source address to an address that will be routed back to the gateway. Messages to the gateway from the Internet have their destination addresses "translated" to the internal address that will route the packet to the correct endnode.

NATs are designed to be "transparent" to endnodes. Neither software on the node behind the NAT nor the node on the Internet requires modification to communicate through the NAT. Achieving this transparency is more difficult with some protocols than with others. Protocols that include IP addresses of the endpoints within the payloads of the packet will fail unless the NAT gateway understands the protocol and modifies the internal references as well as those in the headers. Such knowledge is inherently unreliable, is a network layer violation, and often results in subtle problems.

Opening an IPsec connection through a NAT introduces special problems. If the connection runs in transport mode, changing the IP addresses on packets will cause the checksums to fail and the NAT cannot correct the checksums because they are cryptographically protected. Even in tunnel mode, there are routing problems because transparently translating the addresses of AH and ESP packets requires special logic in the NAT and that logic is heuristic and unreliable in nature. For that reason, IKEv2 can negotiate UDP encapsulation of IKE and ESP packets. This encoding is slightly less efficient but is easier for NATs to process. In addition, firewalls may be configured to pass IPsec traffic over UDP but not ESP/AH or vice versa. 
It is a common practice of NATs to translate TCP and UDP port numbers as well as addresses and use the port numbers of inbound packets to decide which internal node should get a given packet. For this reason, even though IKE packets MUST be sent from and to UDP port 500, they MUST be accepted coming from any port and responses MUST be sent to the port from whence they came. This is because the ports may be modified as the packets pass through NATs. Similarly, IP addresses of the IKE endpoints are generally not included in the IKE payloads because the payloads are cryptographically protected and could not be transparently modified by NATs.

Port 4500 is reserved for UDP-encapsulated ESP and IKE. When working through a NAT, it is generally better to pass IKE packets over port 4500 because some older NATs handle IKE traffic on port 500 cleverly in an attempt to transparently establish IPsec connections between endpoints that don't handle NAT traversal themselves. Such NATs may interfere with the straightforward NAT traversal envisioned by this document, so an IPsec endpoint that discovers a NAT between it and its correspondent MUST send all subsequent traffic to and from port 4500, which NATs should not treat specially (as they might with port $500)$.

The specific requirements for supporting NAT traversal [RFC3715] are listed below. Support for NAT traversal is optional. In this section only, requirements listed as MUST apply only to implementations supporting NAT traversal.

IKE MUST listen on port 4500 as well as port 500. IKE MUST respond to the IP address and port from which packets arrived.

Both IKE initiator and responder MUST include in their IKE_SA_INIT packets Notify payloads of type NAT_DETECTION_SOURCE_IP and NAT_DETECTION_DESTINATION_IP. Those payloads can be used to detect if there is NAT between the hosts, and which end is behind the NAT. The location of the payloads in the IKE_SA_INIT packets are just after the $\mathrm{Ni}$ and $\mathrm{Nr}$ payloads (before the optional CERTREQ payload).

If none of the NAT_DETECTION_SOURCE_IP payload(s) received matches the hash of the source IP and port found from the IP header of the packet containing the payload, it means that the other end is behind NAT (i.e., someone along the route changed the source address of the original packet to match the address of the NAT box). In this case, this end should allow dynamic update of the other ends IP address, as described later. 
If the NAT_DETECTION_DESTINATION_IP payload received does not match the hash of the destination IP and port found from the IP header of the packet containing the payload, it means that this end is behind a NAT. In this case, this end SHOULD start sending keepalive packets as explained in [Hutt05].

The IKE initiator MUST check these payloads if present and if they do not match the addresses in the outer packet MUST tunnel all future IKE and ESP packets associated with this IKE_SA over UDP port 4500 .

To tunnel IKE packets over UDP port 4500, the IKE header has four octets of zero prepended and the result immediately follows the UDP header. To tunnel ESP packets over UDP port 4500, the ESP header immediately follows the UDP header. Since the first four bytes of the ESP header contain the SPI, and the SPI cannot validly be zero, it is always possible to distinguish ESP and IKE messages.

The original source and destination IP address required for the transport mode TCP and UDP packet checksum fixup (see [Hutt05]) are obtained from the Traffic selectors associated with the exchange. In the case of NAT traversal, the Traffic selectors MUST contain exactly one IP address, which is then used as the original IP address.

There are cases where a NAT box decides to remove mappings that are still alive (for example, the keepalive interval is too long, or the NAT box is rebooted). To recover in these cases, hosts that are not behind a NAT SHOULD send all packets (including retransmission packets) to the IP address and port from the last valid authenticated packet from the other end (i.e., dynamically update the address). A host behind a NAT SHOULD NOT do this because it opens a Dos attack possibility. Any authenticated IKE packet or any authenticated UDP-encapsulated ESP packet can be used to detect that the IP address or the port has changed.

Note that similar but probably not identical actions will likely be needed to make IKE work with Mobile IP, but such processing is not addressed by this document.

\subsection{Explicit Congestion Notification (ECN)}

When IPsec tunnels behave as originally specified in [RFC2401], ECN usage is not appropriate for the outer IP headers because tunnel decapsulation processing discards ECN congestion indications to the detriment of the network. ECN support for IPsec tunnels for IKEv1based IPsec requires multiple operating modes and negotiation (see 
[RFC3168]). IKEv2 simplifies this situation by requiring that ECN be usable in the outer IP headers of all tunnel-mode IPsec SAs created by IKEv2. Specifically, tunnel encapsulators and decapsulators for all tunnel-mode SAs created by IKEv2 MUST support the ECN fullfunctionality option for tunnels specified in [RFC3168] and MUST implement the tunnel encapsulation and decapsulation processing specified in [RFC4301] to prevent discarding of ECN congestion indications.

\section{Header and Payload Formats}

\subsection{The IKE Header}

IKE messages use UDP ports 500 and/or 4500, with one IKE message per UDP datagram. Information from the beginning of the packet through the UDP header is largely ignored except that the IP addresses and UDP ports from the headers are reversed and used for return packets. When sent on UDP port 500, IKE messages begin immediately following the UDP header. When sent on UDP port 4500, IKE messages have prepended four octets of zero. These four octets of zero are not part of the IKE message and are not included in any of the length fields or checksums defined by IKE. Each IKE message begins with the IKE header, denoted HDR in this memo. Following the header are one or more IKE payloads each identified by a "Next Payload" field in the preceding payload. Payloads are processed in the order in which they appear in an IKE message by invoking the appropriate processing routine according to the "Next Payload" field in the IKE header and subsequently according to the "Next Payload" field in the IKE payload itself until a "Next Payload" field of zero indicates that no payloads follow. If a payload of type "Encrypted" is found, that payload is decrypted and its contents parsed as additional payloads. An Encrypted payload MUST be the last payload in a packet and an Encrypted payload MUST NOT contain another Encrypted payload.

The Recipient SPI in the header identifies an instance of an IKE security association. It is therefore possible for a single instance of IKE to multiplex distinct sessions with multiple peers.

All multi-octet fields representing integers are laid out in big endian order (aka most significant byte first, or network byte order).

The format of the IKE header is shown in Figure 4. 


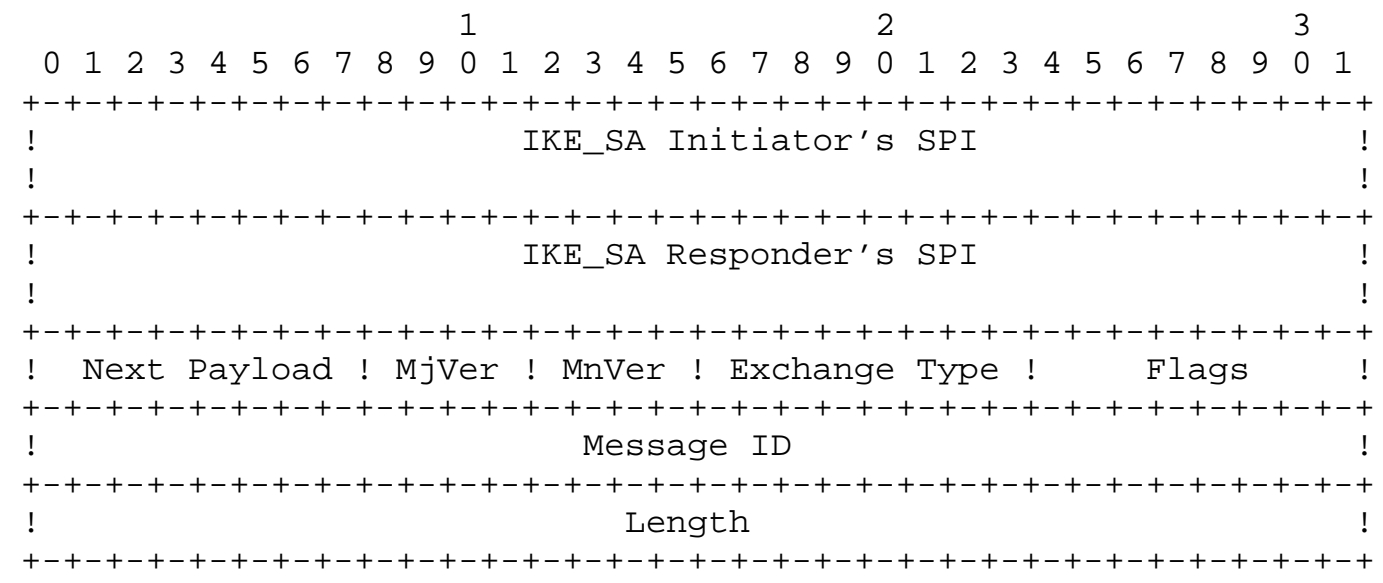

Figure 4: IKE Header Format

- Initiator's SPI (8 octets) - A value chosen by the initiator to identify a unique IKE security association. This value MUST NOT be zero.

- Responder's SPI (8 octets) - A value chosen by the responder to identify a unique IKE security association. This value MUST be zero in the first message of an IKE Initial Exchange (including repeats of that message including a cookie) and MUST NOT be zero in any other message.

- Next Payload (1 octet) - Indicates the type of payload that immediately follows the header. The format and value of each payload are defined below.

- Major Version (4 bits) - Indicates the major version of the IKE protocol in use. Implementations based on this version of IKE MUST set the Major Version to 2. Implementations based on previous versions of IKE and ISAKMP MUST set the Major Version to 1. Implementations based on this version of IKE MUST reject or ignore messages containing a version number greater than 2 .

- Minor Version (4 bits) - Indicates the minor version of the IKE protocol in use. Implementations based on this version of IKE MUST set the Minor Version to 0 . They MUST ignore the minor version number of received messages.

- Exchange Type (1 octet) - Indicates the type of exchange being used. This constrains the payloads sent in each message and orderings of messages in an exchange. 


$\begin{array}{ll}\text { Exchange Type } & \text { Value } \\ \text { RESERVED } & 0-33 \\ \text { IKE_SA_INIT } & 34 \\ \text { IKE_AUTH } & 35 \\ \text { CREATE_CHILD_SA } & 36 \\ \text { INEORMATIONAL } & 37 \\ \text { RESERVED TO IANA } & 38-239 \\ \text { Reserved for private use } & 240-255\end{array}$

- Flags (1 octet) - Indicates specific options that are set for the message. Presence of options are indicated by the appropriate bit in the flags field being set. The bits are defined LSB first, so bit 0 would be the least significant bit of the Flags octet. In the description below, a bit being 'set' means its value is ' 1 ', while 'cleared' means its value is '0'.

-- X(reserved) (bits 0-2) - These bits MUST be cleared when sending and MUST be ignored on receipt.

-- I(nitiator) (bit 3 of Flags) - This bit MUST be set in messages sent by the original initiator of the IKE_SA and MUST be cleared in messages sent by the original responder. It is used by the recipient to determine which eight octets of the SPI were generated by the recipient.

-- V(ersion) (bit 4 of Flags) - This bit indicates that the transmitter is capable of speaking a higher major version number of the protocol than the one indicated in the major version number field. Implementations of IKEv2 must clear this bit when sending and MUST ignore it in incoming messages.

-- R(esponse) (bit 5 of Flags) - This bit indicates that this message is a response to a message containing the same message ID. This bit MUST be cleared in all request messages and MUST be set in all responses. An IKE endpoint MUST NOT generate a response to a message that is marked as being a response.

-- X(reserved) (bits 6-7 of Flags) - These bits MUST be cleared when sending and MUST be ignored on receipt. 


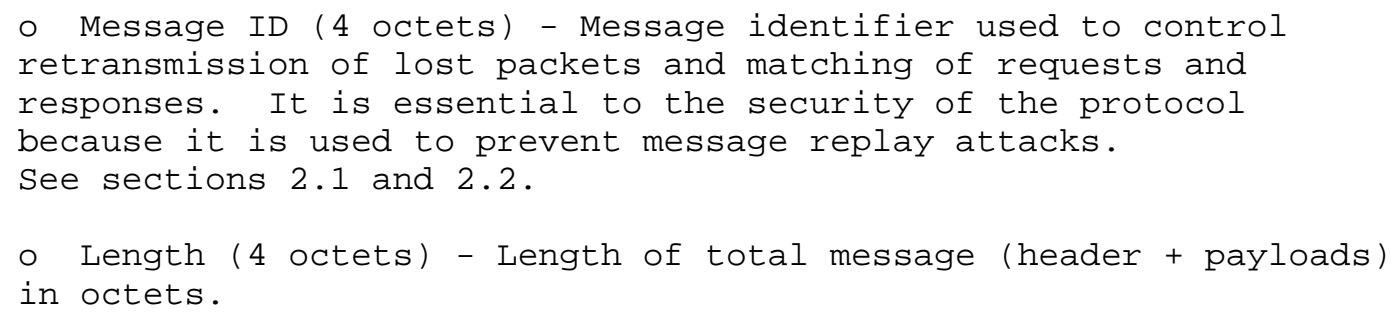

\subsection{Generic Payload Header}

Each IKE payload defined in sections 3.3 through 3.16 begins with a generic payload header, shown in Figure 5. Figures for each payload below will include the generic payload header, but for brevity the description of each field will be omitted.

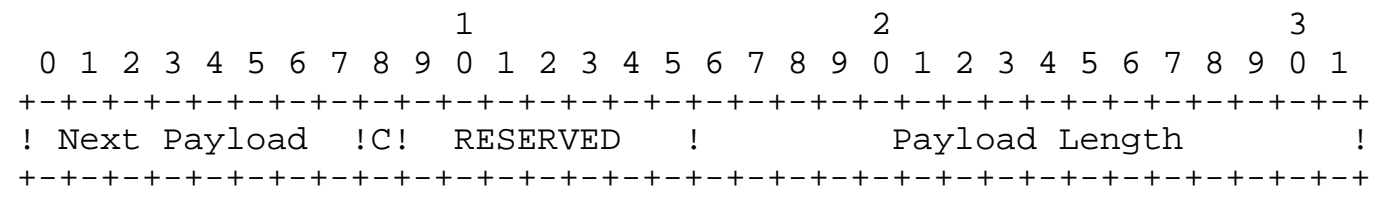

Figure 5: Generic Payload Header

The Generic Payload Header fields are defined as follows:

- Next Payload (1 octet) - Identifier for the payload type of the next payload in the message. If the current payload is the last in the message, then this field will be 0 . This field provides a "chaining" capability whereby additional payloads can be added to a message by appending it to the end of the message and setting the "Next Payload" field of the preceding payload to indicate the new payload's type. An Encrypted payload, which must always be the last payload of a message, is an exception. It contains data structures in the format of additional payloads. In the header of an Encrypted payload, the Next Payload field is set to the payload type of the first contained payload (instead of 0 ).

Payload Type Values

Next Payload Type

Notation Value

No Next Payload

RESERVED

Security Association

Key Exchange

Identification - Initiator

$\begin{array}{lc} & 0 \\ & 1-32 \\ \mathrm{SA} & 33 \\ \mathrm{KE} & 34 \\ \mathrm{ID} i & 35\end{array}$




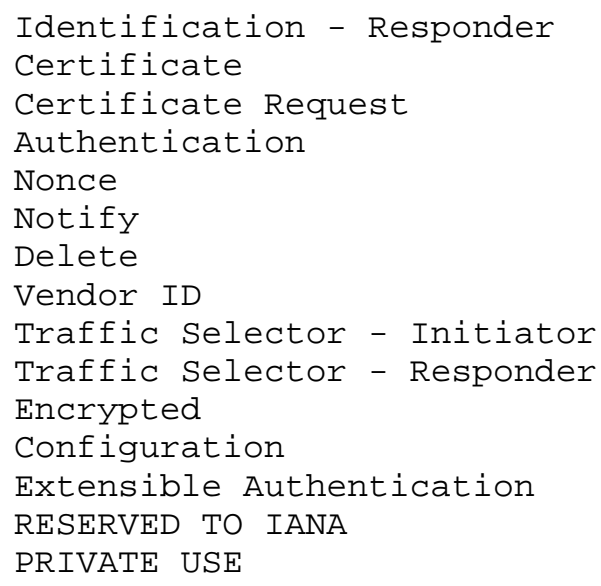

$\begin{array}{lc}\text { IDr } & 36 \\ \text { CERT } & 37 \\ \text { CERTREQ } & 38 \\ \text { AUTH } & 39 \\ \mathrm{Ni}, \mathrm{Nr} & 40 \\ \mathrm{~N} & 41 \\ \mathrm{D} & 42 \\ \mathrm{~V} & 43 \\ \mathrm{TSi} & 44 \\ \mathrm{TSr} & 45 \\ \mathrm{E} & 46 \\ \mathrm{CP} & 47 \\ \text { EAP } & 48 \\ & 49-127 \\ & 128-255\end{array}$

Payload type values $1-32$ should not be used so that there is no overlap with the code assignments for IKEv1. Payload type values 49-127 are reserved to IANA for future assignment in IKEv2 (see section 6). Payload type values $128-255$ are for private use among mutually consenting parties.

- Critical (1 bit) - MUST be set to zero if the sender wants the recipient to skip this payload if it does not understand the payload type code in the Next Payload field of the previous payload. MUST be set to one if the sender wants the recipient to reject this entire message if it does not understand the payload type. MUST be ignored by the recipient if the recipient understands the payload type code. MUST be set to zero for payload types defined in this document. Note that the critical bit applies to the current payload rather than the "next" payload whose type code appears in the first octet. The reasoning behind not setting the critical bit for payloads defined in this document is that all implementations MUST understand all payload types defined in this document and therefore must ignore the Critical bit's value. Skipped payloads are expected to have valid Next Payload and Payload Length fields.

- RESERVED ( 7 bits) - MUST be sent as zero; MUST be ignored on receipt.

- Payload Length (2 octets) - Length in octets of the current payload, including the generic payload header. 


\subsection{Security Association Payload}

The Security Association Payload, denoted SA in this memo, is used to negotiate attributes of a security association. Assembly of Security Association Payloads requires great peace of mind. An SA payload MAY contain multiple proposals. If there is more than one, they MUST be ordered from most preferred to least preferred. Each proposal may contain multiple IPsec protocols (where a protocol is IKE, ESP, or $\mathrm{AH}$, each protocol MAY contain multiple transforms, and each transform MAY contain multiple attributes. When parsing an SA, an implementation MUST check that the total Payload Length is consistent with the payload's internal lengths and counts. Proposals, Transforms, and Attributes each have their own variable length encodings. They are nested such that the Payload Length of an SA includes the combined contents of the SA, Proposal, Transform, and Attribute information. The length of a Proposal includes the lengths of all Transforms and Attributes it contains. The length of a Transform includes the lengths of all Attributes it contains.

The syntax of Security Associations, Proposals, Transforms, and Attributes is based on ISAKMP; however, the semantics are somewhat different. The reason for the complexity and the hierarchy is to allow for multiple possible combinations of algorithms to be encoded in a single SA. Sometimes there is a choice of multiple algorithms, whereas other times there is a combination of algorithms. For example, an initiator might want to propose using ( $\mathrm{AH}$ W/MD5 and ESP w/3DES) OR (ESP w/MD5 and 3DES) .

One of the reasons the semantics of the SA payload has changed from ISAKMP and IKEv1 is to make the encodings more compact in common cases.

The Proposal structure contains within it a Proposal \# and an IPsec protocol ID. Each structure MUST have the same Proposal \# as the previous one or be one (1) greater. The first Proposal MUST have a Proposal \# of one (1). If two successive structures have the same Proposal number, it means that the proposal consists of the first structure AND the second. So a proposal of AH AND ESP would have two proposal structures, one for $A H$ and one for ESP and both would have Proposal \#1. A proposal of AH OR ESP would have two proposal structures, one for AH with Proposal \#1 and one for ESP with Proposal \#2.

Each Proposal/Protocol structure is followed by one or more transform structures. The number of different transforms is generally determined by the Protocol. AH generally has a single transform: an integrity check algorithm. ESP generally has two: an encryption algorithm and an integrity check algorithm. IKE generally has four 
transforms: a Diffie-Hellman group, an integrity check algorithm, a prf algorithm, and an encryption algorithm. If an algorithm that combines encryption and integrity protection is proposed, it MUST be proposed as an encryption algorithm and an integrity protection algorithm MUST NOT be proposed. For each Protocol, the set of permissible transforms is assigned transform ID numbers, which appear in the header of each transform.

If there are multiple transforms with the same Transform Type, the proposal is an OR of those transforms. If there are multiple Transforms with different Transform Types, the proposal is an AND of the different groups. For example, to propose ESP with (3DES or IDEA) and (HMAC_MD5 or HMAC_SHA), the ESP proposal would contain two Transform Type 1 candidates (one for 3DES and one for IDEA) and two Transform Type 2 candidates (one for HMAC_MD5 and one for HMAC_SHA). This effectively proposes four combinations of algorithms. If the initiator wanted to propose only a subset of those, for example (3DES and HMAC_MD5) or (IDEA and HMAC_SHA), there is no way to encode that as multiple transforms within a single Proposal. Instead, the initiator would have to construct two different Proposals, each with two transforms.

A given transform MAY have one or more Attributes. Attributes are necessary when the transform can be used in more than one way, as when an encryption algorithm has a variable key size. The transform would specify the algorithm and the attribute would specify the key size. Most transforms do not have attributes. A transform MUST NOT have multiple attributes of the same type. To propose alternate values for an attribute (for example, multiple key sizes for the AES encryption algorithm), and implementation MUST include multiple Transforms with the same Transform Type each with a single Attribute.

Note that the semantics of Transforms and Attributes are quite different from those in IKEVl. In IKEV1, a single Transform carried multiple algorithms for a protocol with one carried in the Transform and the others carried in the Attributes.

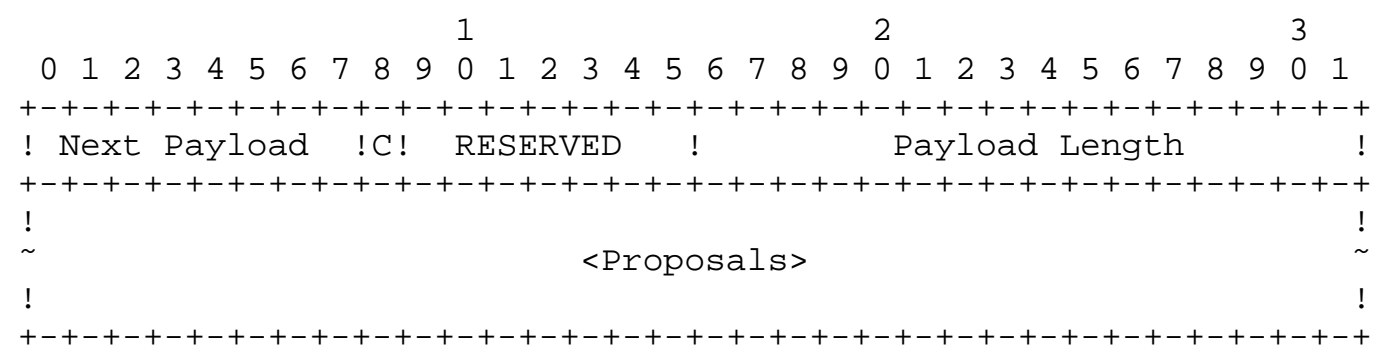

Figure 6: Security Association Payload 
- Proposals (variable) - One or more proposal substructures.

The payload type for the Security Association Payload is thirty three (33).

\subsubsection{Proposal Substructure}

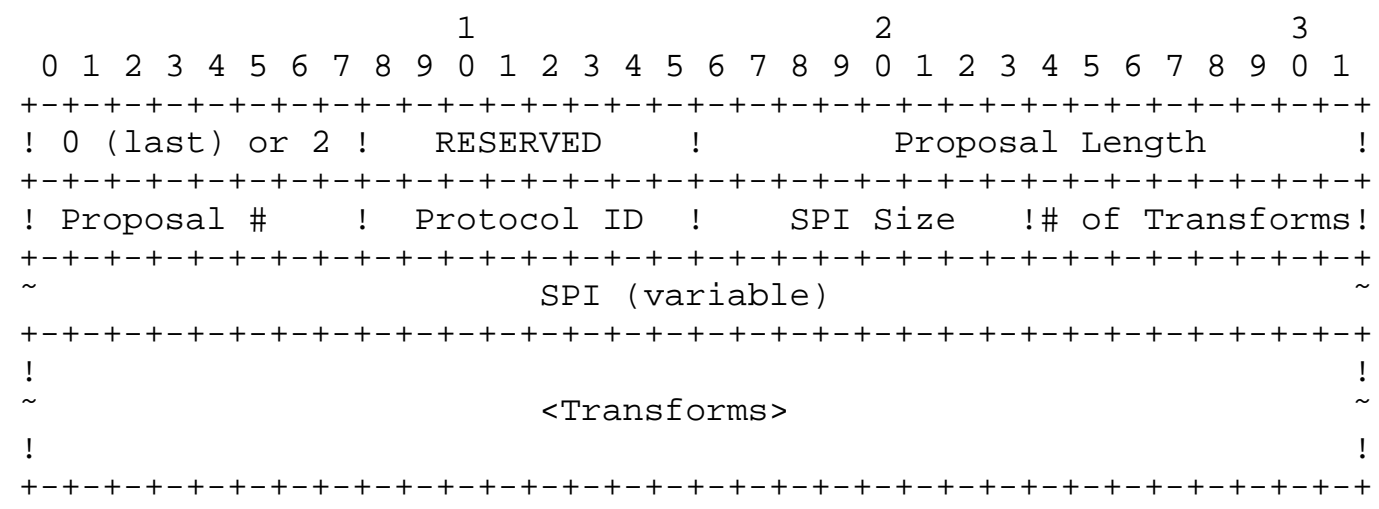

Figure 7: Proposal Substructure

- 0 (last) or 2 (more) (1 octet) - specifies whether this is the last Proposal Substructure in the SA. This syntax is inherited from ISAKMP, but is unnecessary because the last Proposal could be identified from the length of the SA. The value (2) corresponds to a Payload Type of Proposal in IKEv1, and the first 4 octets of the Proposal structure are designed to look somewhat like the header of a Payload.

- RESERVED (1 octet) - MUST be sent as zero; MUST be ignored on receipt.

- Proposal Length (2 octets) - Length of this proposal, including all transforms and attributes that follow.

- Proposal \# (1 octet) - When a proposal is made, the first proposal in an SA payload MUST be \#1, and subsequent proposals MUST either be the same as the previous proposal (indicating an AND of the two proposals) or one more than the previous proposal (indicating an OR of the two proposals). When a proposal is accepted, all of the proposal numbers in the SA payload MUST be the same and MUST match the number on the proposal sent that was accepted. 
- Protocol ID (1 octet) - Specifies the IPsec protocol identifier for the current negotiation. The defined values are:

$\begin{array}{ll}\text { Protocol } & \text { Protocol ID } \\ \text { RESERVED } & 0 \\ \text { IKE } & 1 \\ \text { AH } & 2 \\ \text { ESP } & 3 \\ \text { RESERVED TO IANA } & 4-200 \\ \text { PRIVATE USE } & 201-255\end{array}$

- SPI Size (1 octet) - For an initial IKE_SA negotiation, this field MUST be zero; the SPI is obtained from the outer header. During subsequent negotiations, it is equal to the size, in octets, of the SPI of the corresponding protocol (8 for IKE, 4 for ESP and AH).

o \# of Transforms (1 octet) - Specifies the number of transforms in this proposal.

- SPI (variable) - The sending entity's SPI. Even if the SPI Size is not a multiple of 4 octets, there is no padding applied to the payload. When the SPI Size field is zero, this field is not present in the Security Association payload.

o Transforms (variable) - One or more transform substructures.

3.3.2. Transform Substructure

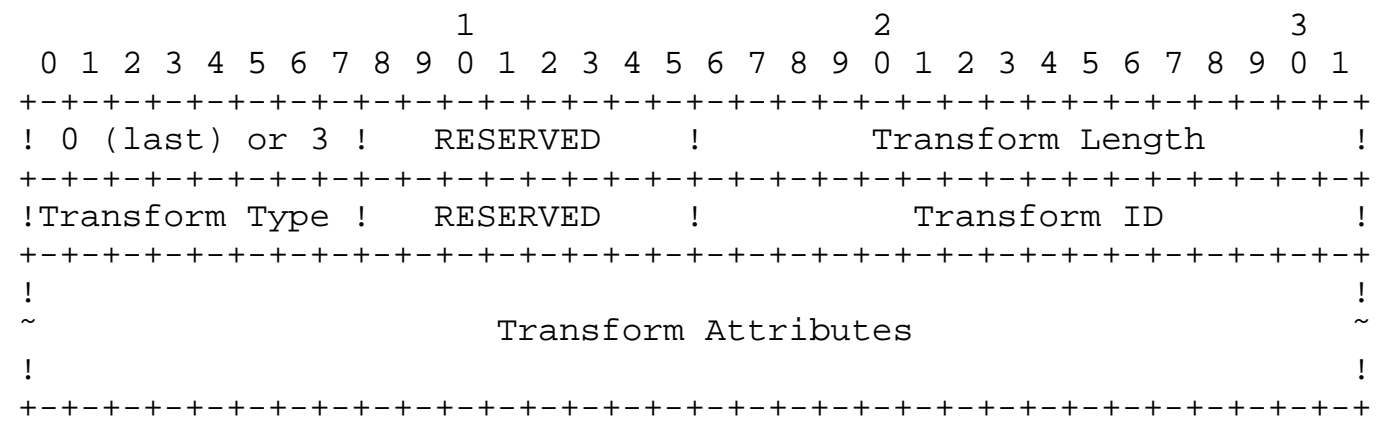

Figure 8: Transform Substructure

- 0 (last) or 3 (more) (1 octet) - specifies whether this is the last Transform Substructure in the Proposal. This syntax is inherited from ISAKMP, but is unnecessary because the last Proposal could be identified from the length of the SA. The 
value (3) corresponds to a Payload Type of Transform in IKEv1, and the first 4 octets of the Transform structure are designed to look somewhat like the header of a Payload.

- RESERVED - MUST be sent as zero; MUST be ignored on receipt.

- Transform Length - The length (in octets) of the Transform Substructure including Header and Attributes.

- Transform Type (1 octet) - The type of transform being specified in this transform. Different protocols support different transform types. For some protocols, some of the transforms may be optional. If a transform is optional and the initiator wishes to propose that the transform be omitted, no transform of the given type is included in the proposal. If the initiator wishes to make use of the transform optional to the responder, it includes a transform substructure with transform ID $=0$ as one of the options.

- Transform ID (2 octets) - The specific instance of the transform type being proposed.

Transform Type Values

RESERVED

Encryption Algorithm (ENCR)

Pseudo-random Function (PRF)

Integrity Algorithm (INTEG)

Diffie-Hellman Group (D-H)

Extended Sequence Numbers

RESERVED TO IANA

PRIVATE USE

\section{Transform Used In}

Type

0

1 (IKE and ESP)

2 (IKE)

3 (IKE, $\mathrm{AH}$, optional in ESP)

4 (IKE, optional in $\mathrm{AH} \& \mathrm{ESP})$

$(\mathrm{ESN}) 5 \quad(\mathrm{AH}$ and $\mathrm{ESP})$

$6-240$

241-255

For Transform Type 1 (Encryption Algorithm), defined Transform IDs are:

\author{
Name \\ RESERVED \\ ENCR_DES_IV64 \\ ENCR_DES \\ ENCR_3DES \\ ENCR_RC5 \\ ENCR_IDEA \\ ENCR_CAST \\ ENCR_BLOWFISH \\ ENCR_3IDEA
}

Number
0
1
2
3
4
5
6
7
8

Defined In

(RFC1827)

(RFC2405), [DES ]

(RFC2451)

(RFC2451)

(RFC2451), [IDEA]

(RFC2451)

(RFC2451)

(RFC2451) 


$\begin{array}{lrr}\text { ENCR_DES_IV32 } & 9 & \\ \text { RESERVED } & 10 & \\ \text { ENCR_NULL } & 11 & \text { (RFC2410) } \\ \text { ENCR_AES_CBC } & 12 & \text { (RFC3602) } \\ \text { ENCR_AES_CTR } & 13 & \text { (RFC3664) }\end{array}$

values 14-1023 are reserved to IANA. Values 1024-65535 are for private use among mutually consenting parties.

For Transform Type 2 (Pseudo-random Function), defined Transform IDs are:

$\begin{array}{lcc}\text { Name } & \text { Number } & \text { Defined In } \\ \text { RESERVED } & 0 & \\ \text { PRF_HMAC_MD5 } & 1 & (\text { RFC2104), [MD5] } \\ \text { PRF_HMAC_SHA1 } & 2 & (\text { RFC2104), [SHA] } \\ \text { PRF_HMAC_TIGER } & 3 & (\text { RFC2104) } \\ \text { PRF_AES128_XCBC } & 4 & \text { (RFC3664) } \\ & & \\ \text { values 5-1023 are reserved to IANA. Values 1024-65535 are for } \\ \text { private use among mutually consenting parties. }\end{array}$

For Transform Type 3 (Integrity Algorithm), defined Transform IDs are:

$\begin{array}{lcc}\text { Name } & \text { Number } & \text { Defined In } \\ \text { NONE } & 0 & \\ \text { AUTH_HMAC_MD5_96 } & 1 & (\text { RFC2403) } \\ \text { AUTH_HMAC_SHA1_96 } & 2 & (\text { RFC2404) } \\ \text { AUTH_DES_MAC } & 3 & \\ \text { AUTH_KPDK_MD5 } & 4 & \\ \text { AUTH_AES_XCBC_96 } & 5 & \\ & & \\ \text { values 6-1023 are reserved to IANA. Values 1024-65535 are for } \\ \text { private use among mutually consenting parties. }\end{array}$

For Transform Type 4 (Diffie-Hellman Group), defined Transform IDs are:

$\begin{array}{ll}\text { Name } & \text { Number } \\ \text { NONE } & 0 \\ \text { Defined in Appendix B } & 1-2 \\ \text { RESERVED } & 3-4 \\ \text { Defined in [ADDGROUP] } & 5 \\ \text { RESERVED TO IANA } & 6-13 \\ \text { Defined in [ADDGROUP] } & 14-18 \\ \text { RESERVED TO IANA } & 19-1023 \\ \text { PRIVATE USE } & 1024-65535\end{array}$


For Transform Type 5 (Extended Sequence Numbers), defined Transform IDs are:

$\begin{array}{ll}\text { Name } & \text { Number } \\ \text { No Extended Sequence Numbers } & 0 \\ \text { Extended Sequence Numbers } & 1 \\ \text { RESERVED } & 2-65535\end{array}$

\subsubsection{Valid Transform Types by Protocol}

The number and type of transforms that accompany an SA payload are dependent on the protocol in the SA itself. An SA payload proposing the establishment of an SA has the following mandatory and optional transform types. A compliant implementation MUST understand all mandatory and optional types for each protocol it supports (though it need not accept proposals with unacceptable suites). A proposal MAY omit the optional types if the only value for them it will accept is NONE .

$\begin{array}{cll}\text { Protocol } & \text { Mandatory Types } & \text { Optional Types } \\ \text { IKE } & \text { ENCR, PRF, INTEG, D-H } & \\ \text { ESP } & \text { ENCR, ESN } & \text { INTEG, D-H } \\ \text { AH } & \text { INTEG, ESN } & \text { D-H }\end{array}$

\subsubsection{Mandatory Transform IDs}

The specification of suites that MUST and SHOULD be supported for interoperability has been removed from this document because they are likely to change more rapidly than this document evolves.

An important lesson learned from IKEv1 is that no system should only implement the mandatory algorithms and expect them to be the best choice for all customers. For example, at the time that this document was written, many IKEv1 implementers were starting to migrate to AES in Cipher Block Chaining (CBC) mode for Virtual Private Network (VPN) applications. Many IPsec systems based on IKEV2 will implement AES, additional Diffie-Hellman groups, and additional hash algorithms, and some IPsec customers already require these algorithms in addition to the ones listed above.

It is likely that IANA will add additional transforms in the future, and some users may want to use private suites, especially for IKE where implementations should be capable of supporting different parameters, up to certain size limits. In support of this goal, all implementations of IKEv2 SHOULD include a management facility that allows specification (by a user or system administrator) of DiffieHellman (DH) parameters (the generator, modulus, and exponent lengths and values) for new DH groups. Implementations SHOULD provide a 
management interface via which these parameters and the associated transform IDs may be entered (by a user or system administrator), to enable negotiating such groups.

All implementations of IKEv2 MUST include a management facility that enables a user or system administrator to specify the suites that are acceptable for use with IKE. Upon receipt of a payload with a set of transform IDs, the implementation MUST compare the transmitted transform IDs against those locally configured via the management controls, to verify that the proposed suite is acceptable based on local policy. The implementation MUST reject SA proposals that are not authorized by these IKE suite controls. Note that cryptographic suites that MUST be implemented need not be configured as acceptable to local policy.

\subsubsection{Transform Attributes}

Each transform in a Security Association payload may include attributes that modify or complete the specification of the transform. These attributes are type/value pairs and are defined below. For example, if an encryption algorithm has a variable-length key, the key length to be used may be specified as an attribute. Attributes can have a value with a fixed two octet length or a variable-length value. For the latter, the attribute is encoded as type/length/value.

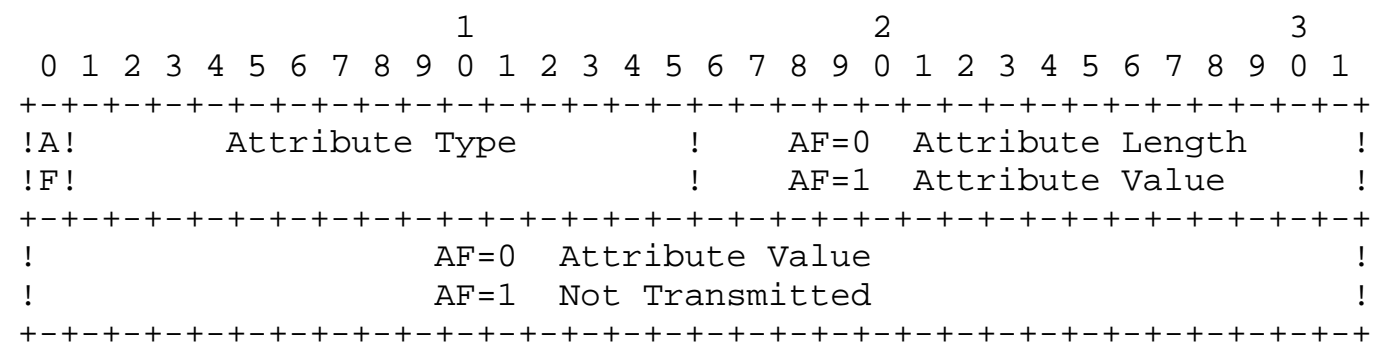

\section{Figure 9: Data Attributes}

- Attribute Type (2 octets) - Unique identifier for each type of attribute (see below).

The most significant bit of this field is the Attribute Format bit $(\mathrm{AF})$. It indicates whether the data attributes follow the Type/Length/Value (TLV) format or a shortened Type/Value (TV) format. If the AF bit is zero (0), then the Data Attributes are of the Type/Length/Value (TLV) form. If the AF bit is a one (1), then the Data Attributes are of the Type/Value form. 
- Attribute Length (2 octets) - Length in octets of the Attribute Value. When the AF bit is a one (1), the Attribute Value is only 2 octets and the Attribute Length field is not present.

- Attribute Value (variable length) - Value of the Attribute associated with the Attribute Type. If the AF bit is a zero (0), this field has a variable length defined by the Attribute Length field. If the $\mathrm{AF}$ bit is a one (1), the Attribute Value has a length of 2 octets.

Note that only a single attribute type (Key Length) is defined, and it is fixed length. The variable-length encoding specification is included only for future extensions. The only algorithms defined in this document that accept attributes are the AES-based encryption, integrity, and pseudo-random functions, which require a single attribute specifying key width.

Attributes described as basic MUST NOT be encoded using the variable-length encoding. Variable-length attributes MUST NOT be encoded as basic even if their value can fit into two octets. NOTE: This is a change from IKEv1, where increased flexibility may have simplified the composer of messages but certainly complicated the parser.

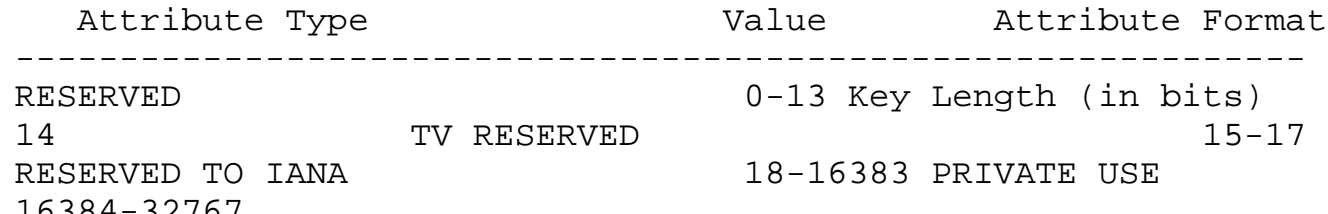

Values 0-13 and 15-17 were used in a similar context in IKEv1 and should not be assigned except to matching values. Values 18-16383 are reserved to IANA. Values 16384-32767 are for private use among mutually consenting parties.

- Key Length

When using an Encryption Algorithm that has a variable-length key, this attribute specifies the key length in bits (MUST use network byte order). This attribute MUST NOT be used when the specified Encryption Algorithm uses a fixed-length key. 


\subsubsection{Attribute Negotiation}

During security association negotiation, initiators present offers to responders. Responders MUST select a single complete set of parameters from the offers (or reject all offers if none are acceptable). If there are multiple proposals, the responder MUST choose a single proposal number and return all of the Proposal substructures with that Proposal number. If there are multiple Transforms with the same type, the responder MUST choose a single one. Any attributes of a selected transform MUST be returned unmodified. The initiator of an exchange MUST check that the accepted offer is consistent with one of its proposals, and if not that response MUST be rejected.

Negotiating Diffie-Hellman groups presents some special challenges. SA offers include proposed attributes and a Diffie-Hellman public number (KE) in the same message. If in the initial exchange the initiator offers to use one of several Diffie-Hellman groups, it SHOULD pick the one the responder is most likely to accept and include a $\mathrm{KE}$ corresponding to that group. If the guess turns out to be wrong, the responder will indicate the correct group in the response and the initiator SHOULD pick an element of that group for its $\mathrm{KE}$ value when retrying the first message. It SHould, however, continue to propose its full supported set of groups in order to prevent a man-in-the-middle downgrade attack.

Implementation Note:

Certain negotiable attributes can have ranges or could have multiple acceptable values. These include the key length of a variable key length symmetric cipher. To further interoperability and to support upgrading endpoints independently, implementers of this protocol SHOULD accept values that they deem to supply greater security. For instance, if a peer is configured to accept a variable-length cipher with a key length of $\mathrm{X}$ bits and is offered that cipher with a larger key length, the implementation SHOULD accept the offer if it supports use of the longer key.

Support of this capability allows an implementation to express a concept of "at least" a certain level of security -- "a key length of _at least_ $\mathrm{X}$ bits for cipher $\mathrm{Y} "$. 


\subsection{Key Exchange Payload}

The Key Exchange Payload, denoted $\mathrm{KE}$ in this memo, is used to exchange Diffie-Hellman public numbers as part of a Diffie-Hellman key exchange. The Key Exchange Payload consists of the IKE generic payload header followed by the Diffie-Hellman public value itself.

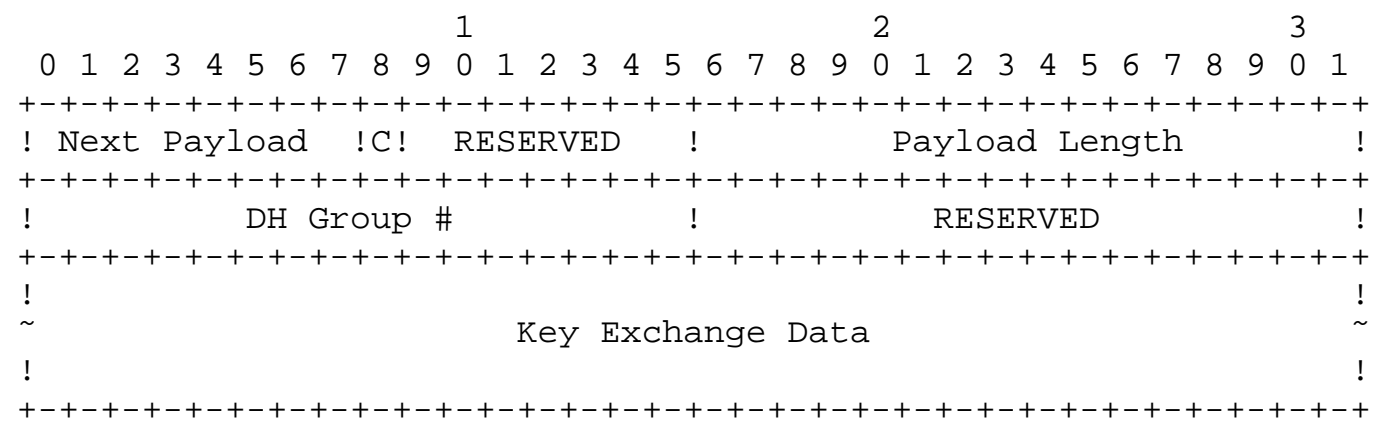

Figure 10: Key Exchange Payload Format

A key exchange payload is constructed by copying one's Diffie-Hellman public value into the "Key Exchange Data" portion of the payload. The length of the Diffie-Hellman public value MUST be equal to the length of the prime modulus over which the exponentiation was performed, prepending zero bits to the value if necessary.

The DH Group \# identifies the Diffie-Hellman group in which the Key Exchange Data was computed (see section 3.3.2). If the selected proposal uses a different Diffie-Hellman group, the message MUST be rejected with a Notify payload of type INVALID_KE_PAYLOAD.

The payload type for the Key Exchange payload is thirty four (34).

\subsection{Identification Payloads}

The Identification Payloads, denoted IDi and IDr in this memo, allow peers to assert an identity to one another. This identity may be used for policy lookup, but does not necessarily have to match anything in the CERT payload; both fields may be used by an implementation to perform access control decisions.

NOTE: In IKEv1, two ID payloads were used in each direction to hold Traffic selector (TS) information for data passing over the SA. In IKEv2, this information is carried in TS payloads (see section 3.13). 
The Identification Payload consists of the IKE generic payload header followed by identification fields as follows:

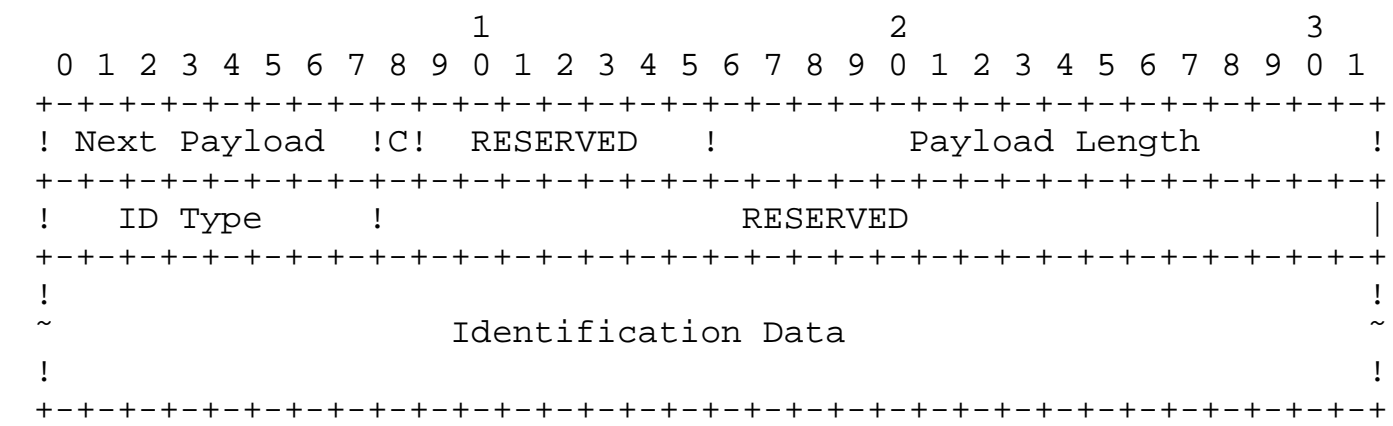

Figure 11: Identification Payload Format

- ID Type (1 octet) - Specifies the type of Identification being used.

- RESERVED - MUST be sent as zero; MUST be ignored on receipt.

- Identification Data (variable length) - Value, as indicated by the Identification Type. The length of the Identification Data is computed from the size in the ID payload header.

The payload types for the Identification Payload are thirty five (35) for IDi and thirty six (36) for IDr.

The following table lists the assigned values for the Identification Type field, followed by a description of the Identification Data which follows:

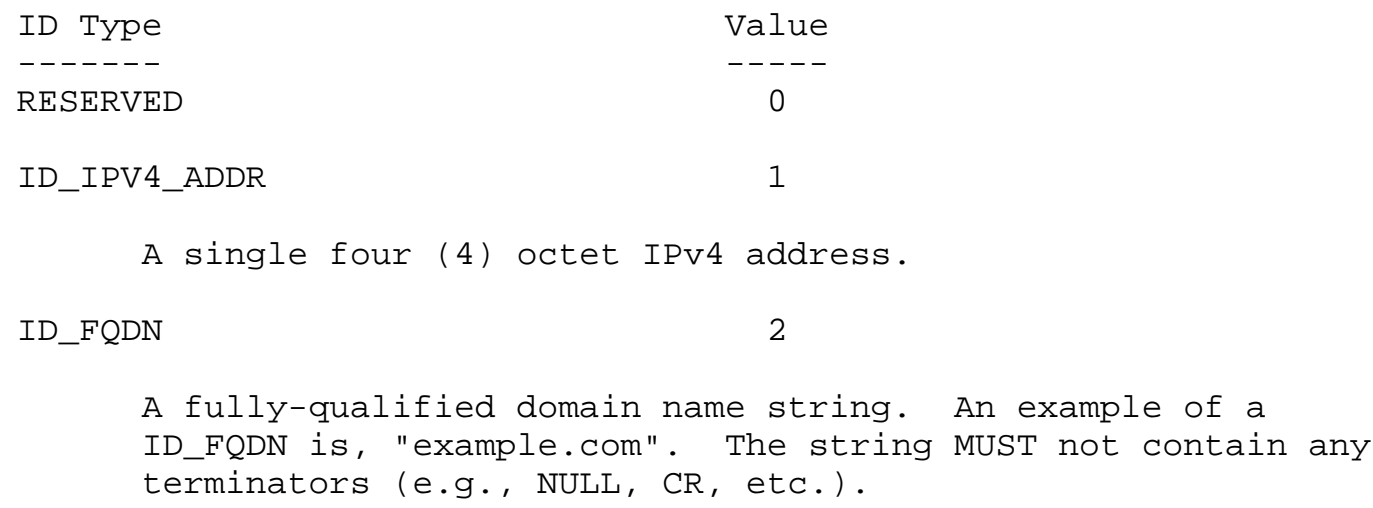




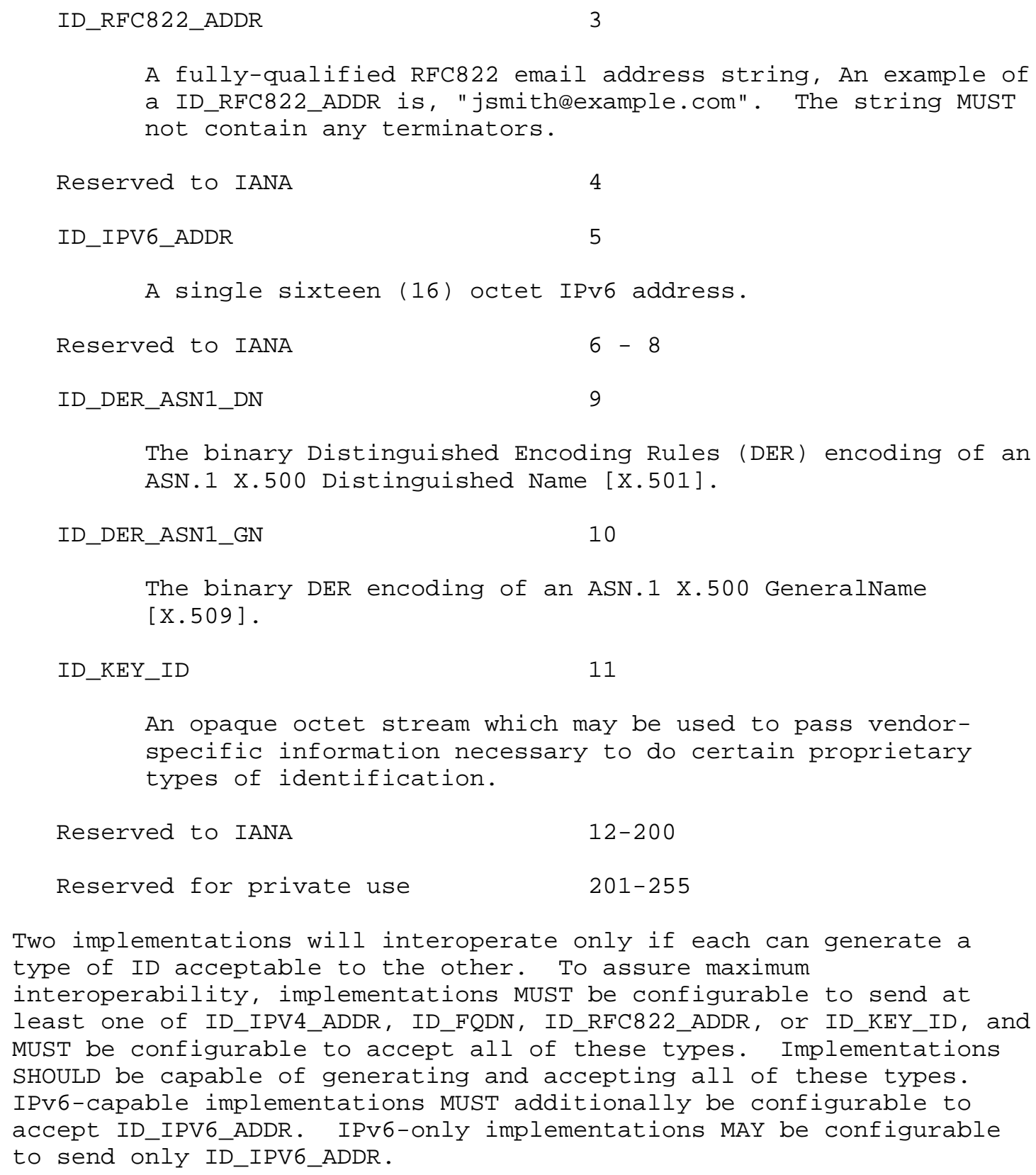

A fully-qualified RFC822 email address string, An example of a ID_RFC822_ADDR is, "jsmith@example.com". The string MUST not contain any terminators. 


\subsection{Certificate Payload}

The Certificate Payload, denoted CERT in this memo, provides a means to transport certificates or other authentication-related information via IKE. Certificate payloads SHOULD be included in an exchange if certificates are available to the sender unless the peer has indicated an ability to retrieve this information from elsewhere using an HTTP_CERT_LOOKUP_SUPPORTED Notify payload. Note that the term "Certificate Payload" is somewhat misleading, because not all authentication mechanisms use certificates and data other than certificates may be passed in this payload.

The Certificate Payload is defined as follows:

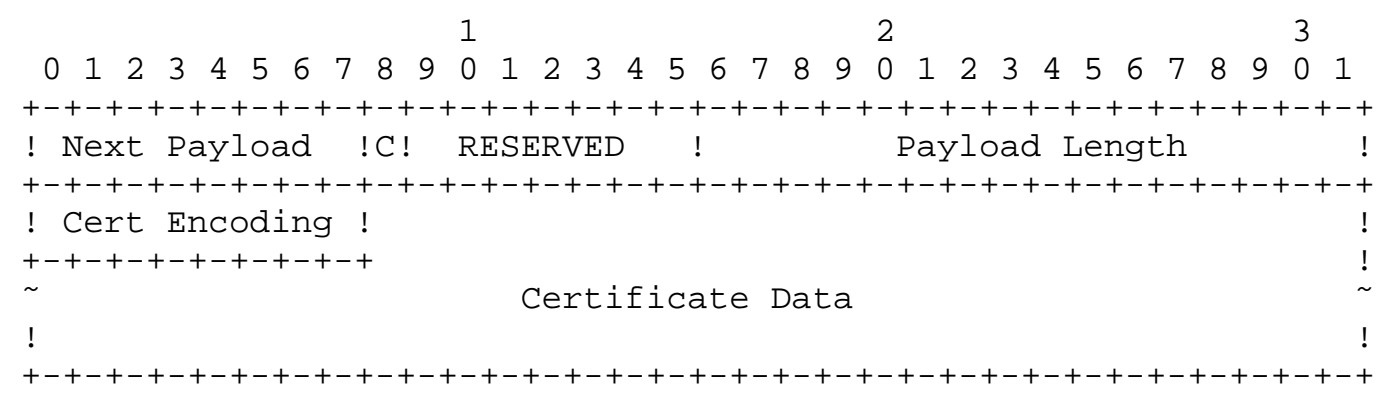

Figure 12: Certificate Payload Format

- Certificate Encoding (1 octet) - This field indicates the type of certificate or certificate-related information contained in the Certificate Data field.

$\begin{array}{lc}\text { Certificate Encoding } & \text { Value } \\ ------ \\ \text { RESERVED } & 0 \\ \text { PKCS \#7 wrapped X.509 certificate } & 1 \\ \text { PGP Certificate } & 2 \\ \text { DNS Signed Key } & 3 \\ \text { X.509 Certificate - Signature } & 4 \\ \text { Kerberos Token } & 6 \\ \text { Certificate Revocation List (CRL) } & 7 \\ \text { Authority Revocation List (ARL) } & 8 \\ \text { SPKI Certificate } & 9 \\ \text { X.509 Certificate - Attribute } & 10 \\ \text { Raw RSA Key } & 11 \\ \text { Hash and URL of X.509 certificate } & 12 \\ \text { Hash and URL of X.509 bundle } & 13 \\ \text { RESERVED to IANA } & 14-200 \\ \text { PRIVATE USE } & 201-255\end{array}$


- Certificate Data (variable length) - Actual encoding of certificate data. The type of certificate is indicated by the Certificate Encoding field.

The payload type for the Certificate Payload is thirty seven (37). Specific syntax is for some of the certificate type codes above is not defined in this document. The types whose syntax is defined in this document are:

X.509 Certificate - Signature (4) contains a DER encoded X.509 certificate whose public key is used to validate the sender's AUTH payload.

Certificate Revocation List (7) contains a DER encoded X.509 certificate revocation list.

Raw RSA Key (11) contains a PKCS \#1 encoded RSA key (see [RSA] and [PKCS1]).

Hash and URL encodings (12-13) allow IKE messages to remain short by replacing long data structures with a 20 octet SHA-1 hash (see [SHA]) of the replaced value followed by a variable-length URL that resolves to the DER encoded data structure itself. This improves efficiency when the endpoints have certificate data cached and makes IKE less subject to denial of service attacks that become easier to mount when IKE messages are large enough to require IP fragmentation [KPS03].

Use the following ASN.1 definition for an X.509 bundle:

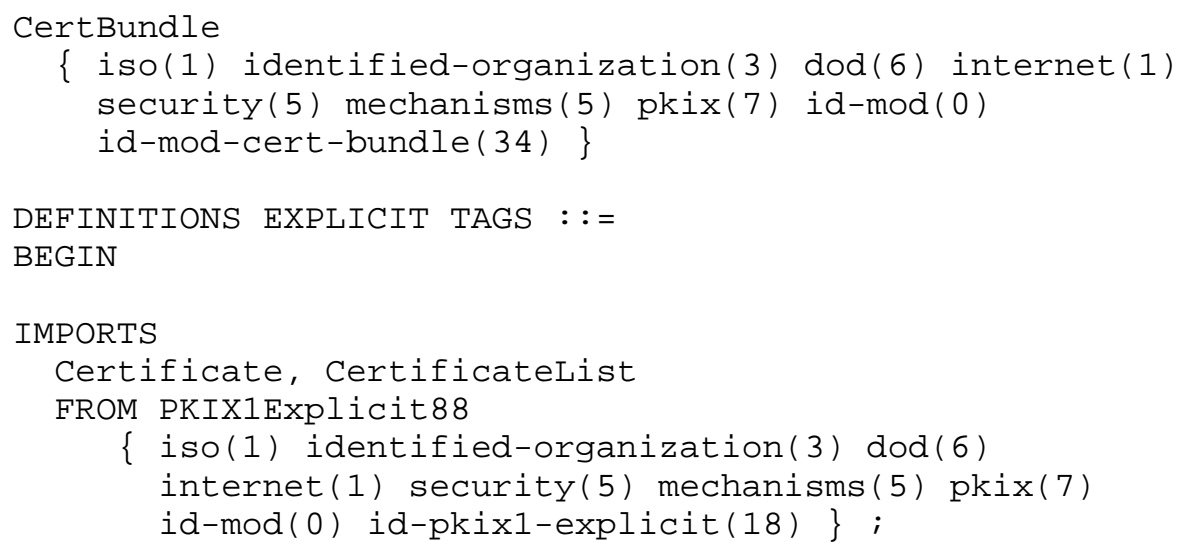




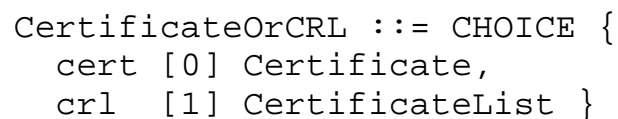

CertificateBundle : := SEQUENCE OF CertificateOrCRL

END

Implementations MUST be capable of being configured to send and accept up to four X.509 certificates in support of authentication, and also MUST be capable of being configured to send and accept the first two Hash and URL formats (with HTTP URLs). Implementations SHOULD be capable of being configured to send and accept Raw RSA keys. If multiple certificates are sent, the first certificate MUST contain the public key used to sign the AUTH payload. The other certificates may be sent in any order.

\subsection{Certificate Request Payload}

The Certificate Request Payload, denoted CERTREQ in this memo, provides a means to request preferred certificates via IKE and can appear in the IKE_INIT_SA response and/or the IKE_AUTH request. Certificate Request payloads MAY be included in an exchange when the sender needs to get the certificate of the receiver. If multiple CAs are trusted and the cert encoding does not allow a list, then multiple Certificate Request payloads SHOULD be transmitted.

The Certificate Request Payload is defined as follows:

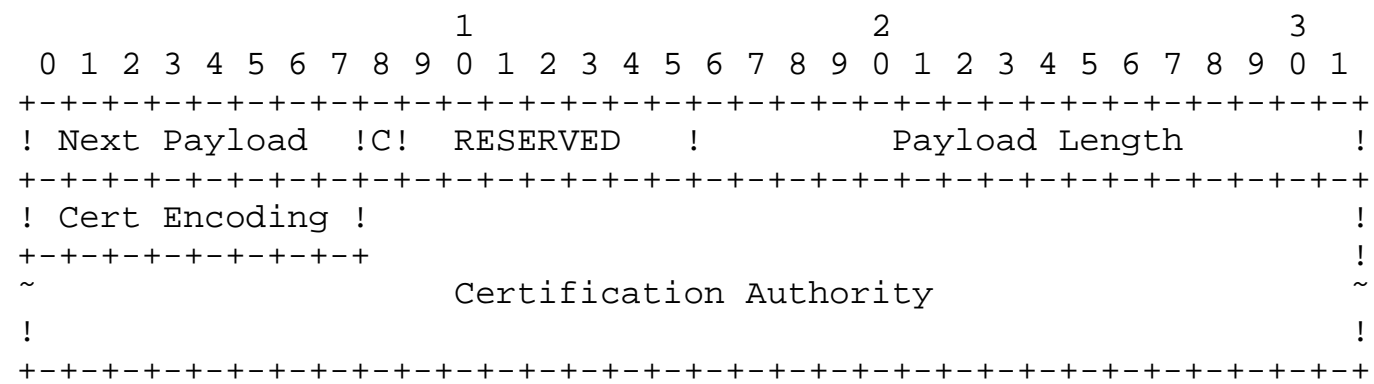

Figure 13: Certificate Request Payload Format

- Certificate Encoding (1 octet) - Contains an encoding of the type or format of certificate requested. Values are listed in section 3.6 . 
- Certification Authority (variable length) - Contains an encoding of an acceptable certification authority for the type of certificate requested.

The payload type for the Certificate Request Payload is thirty eight (38).

The Certificate Encoding field has the same values as those defined in section 3.6. The Certification Authority field contains an indicator of trusted authorities for this certificate type. The Certification Authority value is a concatenated list of SHA-1 hashes of the public keys of trusted Certification Authorities (CAs). Each is encoded as the SHA-1 hash of the Subject Public Key Info element (see section 4.1.2.7 of [RFC3280]) from each Trust Anchor certificate. The twenty-octet hashes are concatenated and included with no other formatting.

Note that the term "Certificate Request" is somewhat misleading, in that values other than certificates are defined in a "Certificate" payload and requests for those values can be present in a Certificate Request Payload. The syntax of the Certificate Request payload in such cases is not defined in this document.

The Certificate Request Payload is processed by inspecting the "Cert Encoding" field to determine whether the processor has any certificates of this type. If so, the "Certification Authority" field is inspected to determine if the processor has any certificates that can be validated up to one of the specified certification authorities. This can be a chain of certificates.

If an end-entity certificate exists that satisfies the criteria specified in the CERTREQ, a certificate or certificate chain SHOULD be sent back to the certificate requestor if the recipient of the CERTREQ :

- is configured to use certificate authentication,

- is allowed to send a CERT payload,

- has matching CA trust policy governing the current negotiation, and

- has at least one time-wise and usage appropriate end-entity certificate chaining to a CA provided in the CERTREQ.

Certificate revocation checking must be considered during the chaining process used to select a certificate. Note that even if two peers are configured to use two different CAs, cross-certification relationships should be supported by appropriate selection logic. 
The intent is not to prevent communication through the strict adherence of selection of a certificate based on CERTREQ, when an alternate certificate could be selected by the sender that would still enable the recipient to successfully validate and trust it through trust conveyed by cross-certification, CRLs, or other outof-band configured means. Thus, the processing of a CERTREQ should be seen as a suggestion for a certificate to select, not a mandated one. If no certificates exist, then the CERTREQ is ignored. This is not an error condition of the protocol. There may be cases where there is a preferred CA sent in the CERTREQ, but an alternate might be acceptable (perhaps after prompting a human operator).

\subsection{Authentication Payload}

The Authentication Payload, denoted AUTH in this memo, contains data used for authentication purposes. The syntax of the Authentication data varies according to the Auth Method as specified below.

The Authentication Payload is defined as follows:

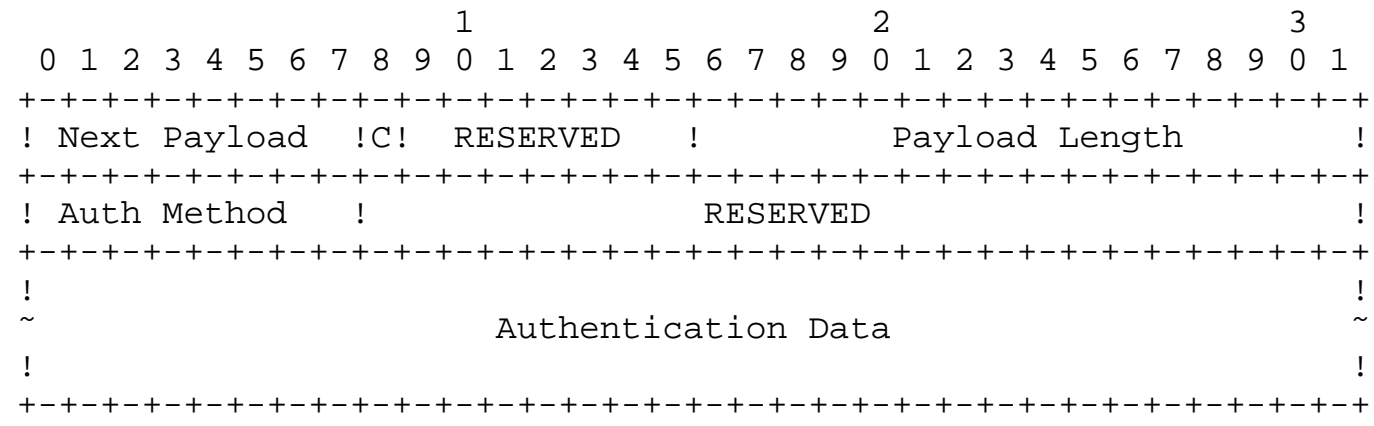

\section{Figure 14: Authentication Payload Format}

- Auth Method (1 octet) - Specifies the method of authentication used. Values defined are:

RSA Digital Signature (1) - Computed as specified in section 2.15 using an RSA private key over a PKCS\#1 padded hash (see [RSA] and [PKCS1]).

Shared Key Message Integrity Code (2) - Computed as specified in section 2.15 using the shared key associated with the identity in the ID payload and the negotiated prf function

DSS Digital Signature (3) - Computed as specified in section 2.15 using a DSS private key (see [DSS]) over a SHA-1 hash. 
The values 0 and 4-200 are reserved to IANA. The values 201-255 are available for private use.

o Authentication Data (variable length) - see section 2.15.

The payload type for the Authentication Payload is thirty nine (39).

3.9. Nonce Payload

The Nonce Payload, denoted $\mathrm{Ni}$ and $\mathrm{Nr}$ in this memo for the initiator's and responder's nonce respectively, contains random data used to guarantee liveness during an exchange and protect against replay attacks.

The Nonce Payload is defined as follows:

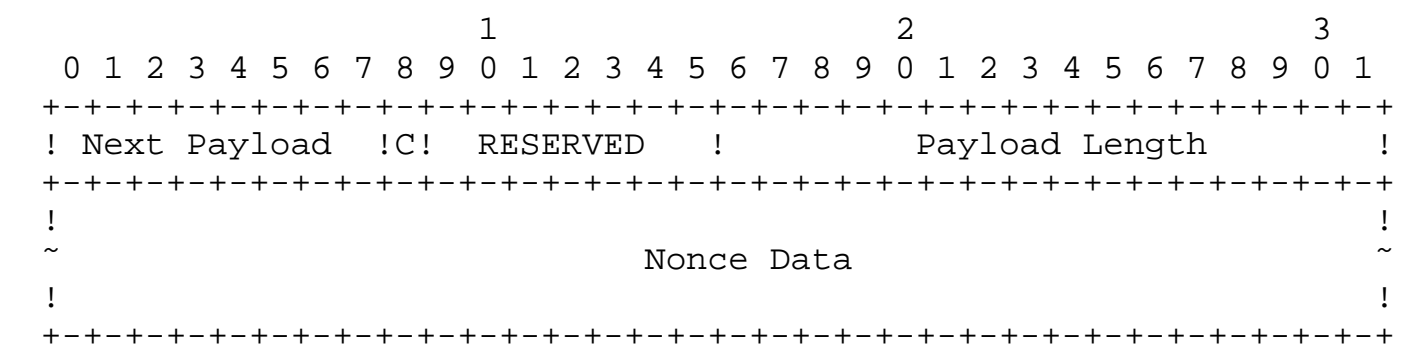

\section{Figure 15: Nonce Payload Format}

- Nonce Data (variable length) - Contains the random data generated by the transmitting entity.

The payload type for the Nonce Payload is forty (40).

The size of a Nonce MUST be between 16 and 256 octets inclusive. Nonce values MUST NOT be reused.

\subsection{Notify Payload}

The Notify Payload, denoted $\mathrm{N}$ in this document, is used to transmit informational data, such as error conditions and state transitions, to an IKE peer. A Notify Payload may appear in a response message (usually specifying why a request was rejected), in an INFORMATIONAL Exchange (to report an error not in an IKE request), or in any other message to indicate sender capabilities or to modify the meaning of the request. 
The Notify Payload is defined as follows:

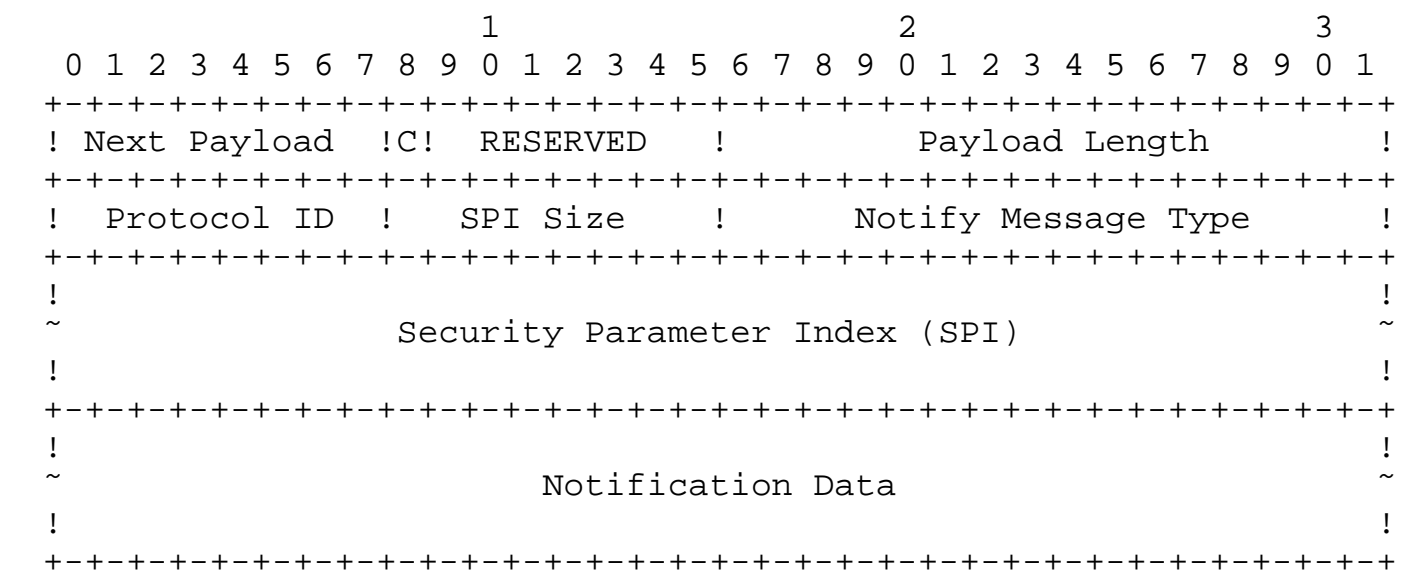

Figure 16: Notify Payload Format

- Protocol ID (1 octet) - If this notification concerns an existing SA, this field indicates the type of that SA. For IKE_SA notifications, this field MUST be one (1). For notifications concerning IPsec SAs this field MUST contain either (2) to indicate $\mathrm{AH}$ or (3) to indicate ESP. For notifications that do not relate to an existing SA, this field MUST be sent as zero and MUST be ignored on receipt. All other values for this field are reserved to IANA for future assignment.

- SPI Size (1 octet) - Length in octets of the SPI as defined by the IPsec protocol ID or zero if no SPI is applicable. For a notification concerning the IKE_SA, the SPI Size MUST be zero.

- Notify Message Type (2 octets) - Specifies the type of notification message.

- SPI (variable length) - Security Parameter Index.

- Notification Data (variable length) - Informational or error data transmitted in addition to the Notify Message Type. Values for this field are type specific (see below).

The payload type for the Notify Payload is forty one (41). 


\subsubsection{Notify Message Types}

Notification information can be error messages specifying why an SA could not be established. It can also be status data that a process managing an SA database wishes to communicate with a peer process. The table below lists the Notification messages and their corresponding values. The number of different error statuses was greatly reduced from IKEv1 both for simplification and to avoid giving configuration information to probers.

Types in the range 0 - 16383 are intended for reporting errors. An implementation receiving a Notify payload with one of these types that it does not recognize in a response MUST assume that the corresponding request has failed entirely. Unrecognized error types in a request and status types in a request or response MUST be ignored except that they SHOULD be logged.

Notify payloads with status types MAY be added to any message and MUST be ignored if not recognized. They are intended to indicate capabilities, and as part of SA negotiation are used to negotiate non-cryptographic parameters.

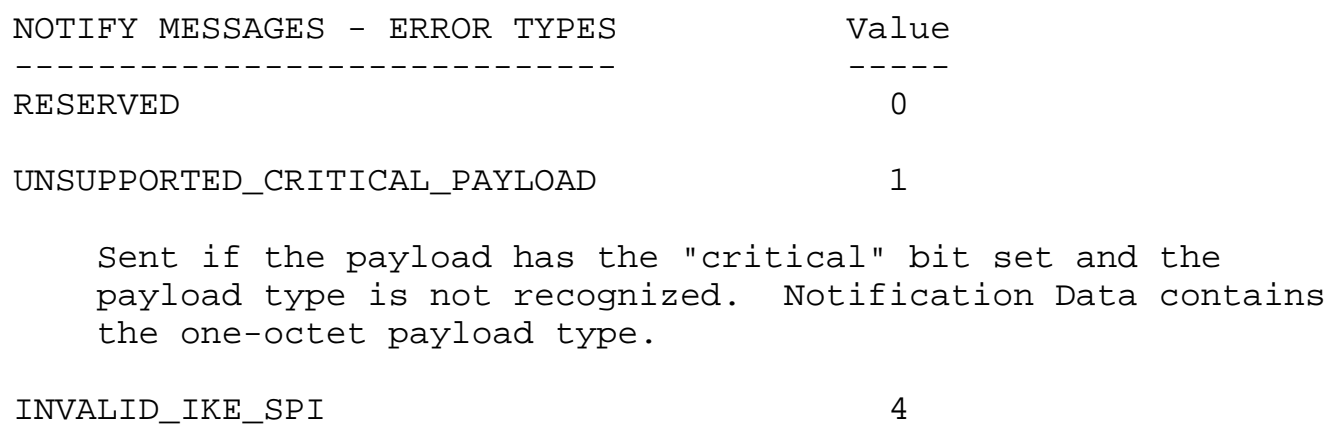


because the request was rejected for policy reasons. To avoid a denial of service attack using forged messages, this status may only be returned for and in an encrypted packet if the message ID and cryptographic checksum were valid. To avoid leaking information to someone probing a node, this status MUST be sent in response to any error not covered by one of the other status types. To aid debugging, more detailed error information SHOULD be written to a console or $\log$.

INVALID_MESSAGE_ID

9

Sent when an IKE message ID outside the supported window is received. This Notify MUST NOT be sent in a response; the invalid request MUST NOT be acknowledged. Instead, inform the other side by initiating an INFORMATIONAL exchange with Notification data containing the four octet invalid message ID. Sending this notification is optional, and notifications of this type MUST be rate limited.

INVALID_SPI

11

MAY be sent in an IKE INFORMATIONAL exchange when a node receives an ESP or AH packet with an invalid SPI. The Notification Data contains the SPI of the invalid packet. This usually indicates a node has rebooted and forgotten an SA. If this Informational Message is sent outside the context of an IKE_SA, it should be used by the recipient only as a "hint" that something might be wrong (because it could easily be forged).

NO_PROPOSAL_CHOSEN

None of the proposed crypto suites was acceptable.

$\begin{array}{ll}\text { INVALID_KE_PAYLOAD } & 17\end{array}$

The D-H Group \# field in the $\mathrm{KE}$ payload is not the group \# selected by the responder for this exchange. There are two octets of data associated with this notification: the accepted D-H Group \# in big endian order.

AUTHENTICATION_FAILED

24

Sent in the response to an IKE_AUTH message when for some reason the authentication failed. There is no associated data. 
This error indicates that a CREATE_CHILD_SA request is unacceptable because its sender is only willing to accept traffic selectors specifying a single pair of addresses. The requestor is expected to respond by requesting an SA for only the specific traffic it is trying to forward.

NO_ADDITIONAL_SAS

35

This error indicates that a CREATE_CHILD_SA request is unacceptable because the responder is unwilling to accept any more CHILD_SAs on this IKE_SA. Some minimal implementations may only accept a single CHILD_SA setup in the context of an initial IKE exchange and reject any subsequent attempts to add more.

INTERNAL_ADDRESS_FAILURE

36

Indicates an error assigning an internal address (i.e., INTERNAL_IP4_ADDRESS Or INTERNAL_IP6_ADDRESS) during the processing of a Configuration Payload by a responder. If this error is generated within an IKE_AUTH exchange, no CHILD_SA will be created.

FAILED_CP_REQUIRED

Sent by responder in the case where CP (CFG_REQUEST) was expected but not received, and so is a conflict with locally configured policy. There is no associated data.

TS_UNACCEPTABLE 38

Indicates that none of the addresses/protocols/ports in the supplied traffic selectors is acceptable.

INVALID_SELECTORS 39

MAY be sent in an IKE INFORMATIONAL exchange when a node receives an ESP or AH packet whose selectors do not match those of the SA on which it was delivered (and that caused the packet to be dropped). The Notification Data contains the start of the offending packet (as in ICMP messages) and the SPI field of the notification is set to match the SPI of the IPsec SA.

RESERVED TO IANA - Error types 40 - 8191

Private Use - Errors $\quad 8192$ - 16383 
NOTIFY MESSAGES - STATUS TYPES Value

------------------------------ -----

INITIAL_CONTACT

16384

This notification asserts that this IKE_SA is the only IKE_SA currently active between the authenticated identities. It MAY be sent when an IKE_SA is established after a crash, and the recipient MAY use this information to delete any other IKE_SAs it has to the same authenticated identity without waiting for a timeout. This notification MUST NOT be sent by an entity that may be replicated (e.g., a roaming user's credentials where the user is allowed to connect to the corporate firewall from two remote systems at the same time).

SET_WINDOW_SIZE

16385

This notification asserts that the sending endpoint is capable of keeping state for multiple outstanding exchanges, permitting the recipient to send multiple requests before getting a response to the first. The data associated with a SET_WINDOW_SIZE notification MUST be 4 octets long and contain the big endian representation of the number of messages the sender promises to keep. Window size is always one until the initial exchanges complete.

ADDITIONAL_TS_POSSIBLE

16386

This notification asserts that the sending endpoint narrowed the proposed traffic selectors but that other traffic selectors would also have been acceptable, though only in a separate SA (see section 2.9). There is no data associated with this Notify type. It may be sent only as an additional payload in a message including accepted TSs.

IPCOMP_SUPPORTED

16387

This notification may be included only in a message containing an SA payload negotiating a CHILD_SA and indicates a willingness by its sender to use IPComp on this SA. The data associated with this notification includes a two-octet IPComp CPI followed by a one-octet transform ID optionally followed by attributes whose length and format are defined by that transform ID. A message proposing an SA may contain multiple IPCOMP_SUPPORTED notifications to indicate multiple supported algorithms. A message accepting an SA may contain at most one. 
The transform IDs currently defined are:

$\begin{array}{lcl}\text { NAME } & \text { NUMBER } & \text { DEFINED IN } \\ ---------- & ------ & --------- \\ \text { RESERVED } & 0 & \\ \text { IPCOMP_OUI } & 1 & \text { RFC 2394 } \\ \text { IPCOMP_DEFLATE } & 2 & \text { RFC 2395 } \\ \text { IPCOMP_LZS } & 3 & \text { RFC 3051 } \\ \text { IPCOMP_LZJH } & 4 & \\ & & \\ \text { values 5-240 are reserved to IANA. Values 241-255 are } \\ \text { for private use among mutually consenting parties. }\end{array}$

NAT_DETECTION_SOURCE_IP 16388

This notification is used by its recipient to determine whether the source is behind a NAT box. The data associated with this notification is a SHA-1 digest of the SPIs (in the order they appear in the header), IP address, and port on which this packet was sent. There MAY be multiple Notify payloads of this type in a message if the sender does not know which of several network attachments will be used to send the packet. The recipient of this notification MAY compare the supplied value to a SHA-1 hash of the SPIs, source IP address, and port, and if they don't match it SHOULD enable NAT traversal (see section 2.23). Alternately, it MAY reject the connection attempt if NAT traversal is not supported.

NAT_DETECTION_DESTINATION_IP

This notification is used by its recipient to determine whether it is behind a NAT box. The data associated with this notification is a SHA-1 digest of the SPIs (in the order they appear in the header), IP address, and port to which this packet was sent. The recipient of this notification MAY compare the supplied value to a hash of the SPIs, destination IP address, and port, and if they don't match it SHOULD invoke NAT traversal (see section 2.23). If they don't match, it means that this end is behind a NAT and this end SHOULD start sending keepalive packets as defined in [Hutt05]. Alternately, it MAY reject the connection attempt if NAT traversal is not supported. 
COOKIE

16390

This notification MAY be included in an IKE_SA_INIT

response. It indicates that the request should be retried with a copy of this notification as the first payload. This notification MUST be included in an IKE_SA_INIT request retry if a COOKIE notification was included in the initial response. The data associated with this notification MUST be between 1 and 64 octets in length (inclusive).

USE_TRANSPORT_MODE

16391

This notification MAY be included in a request message that also includes an SA payload requesting a CHILD_SA. It requests that the CHILD_SA use transport mode rather than tunnel mode for the SA created. If the request is accepted, the response MUST also include a notification of type USE_TRANSPORT_MODE. If the responder declines the request, the CHILD_SA will be established in tunnel mode. If this is unacceptable to the initiator, the initiator MUST delete the SA. Note: Except when using this option to negotiate transport mode, all CHILD_SAs will use tunnel mode.

Note: The ECN decapsulation modifications specified in [RFC4301] MUST be performed for every tunnel mode SA created by IKEV2.

HTTP_CERT_LOOKUP_SUPPORTED

16392

This notification MAY be included in any message that can include a CERTREQ payload and indicates that the sender is capable of looking up certificates based on an HTTP-based URL (and hence presumably would prefer to receive certificate specifications in that format).

REKEY_SA

16393

This notification MUST be included in a CREATE_CHILD_SA exchange if the purpose of the exchange is to replace an existing ESP or AH SA. The SPI field identifies the SA being rekeyed. There is no data.

ESP_TFC_PADDING_NOT_SUPPORTED 16394

This notification asserts that the sending endpoint will NOT accept packets that contain Flow Confidentiality (TFC) padding. 
NON_FIRST_FRAGMENTS_ALSO

Used for fragmentation control. See [RFC4301] for explanation.

$$
\begin{array}{lr}
\text { RESERVED TO IANA - STATUS TYPES } & 16396 \text { - } 40959 \\
\text { Private Use - STATUS TYPES } & 40960 \text { - } 65535
\end{array}
$$

\subsection{Delete Payload}

The Delete Payload, denoted D in this memo, contains a protocolspecific security association identifier that the sender has removed from its security association database and is, therefore, no longer valid. Figure 17 shows the format of the Delete Payload. It is possible to send multiple SPIs in a Delete payload; however, each SPI MUST be for the same protocol. Mixing of protocol identifiers MUST NOT be performed in a Delete payload. It is permitted, however, to include multiple Delete payloads in a single INFORMATIONAL exchange where each Delete payload lists SPIs for a different protocol.

Deletion of the IKE_SA is indicated by a protocol ID of 1 (IKE) but no SPIs. Deletion of a CHILD_SA, such as ESP or AH, will contain the IPsec protocol ID of that protocol (2 for AH, 3 for ESP), and the SPI is the SPI the sending endpoint would expect in inbound ESP or $A H$ packets.

The Delete Payload is defined as follows:

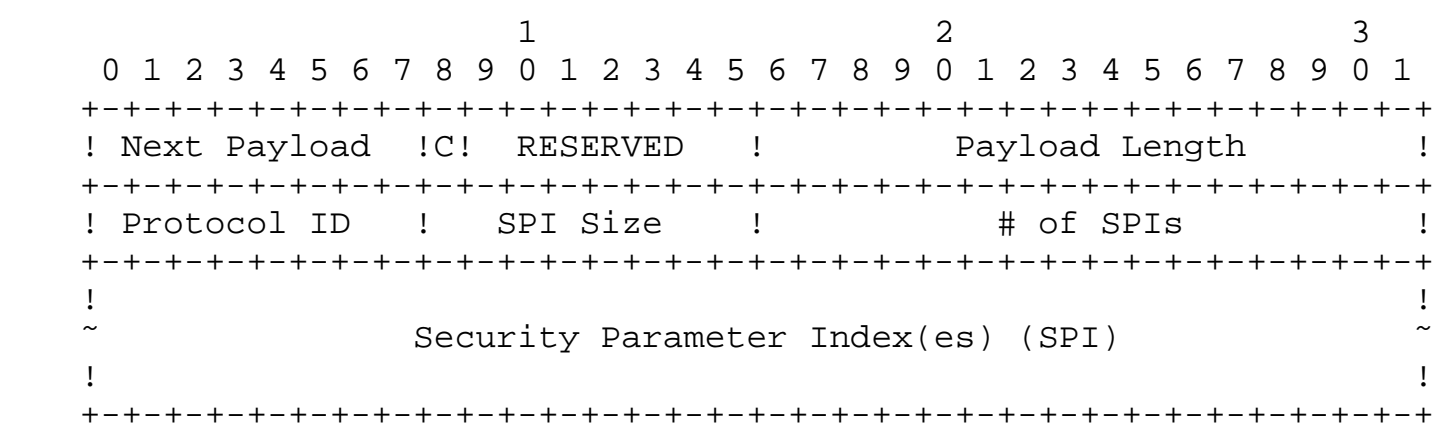

Figure 17: Delete Payload Format

o Protocol ID (1 octet) - Must be 1 for an IKE_SA, 2 for AH, or 3 for ESP. 
- SPI Size (1 octet) - Length in octets of the SPI as defined by the protocol ID. It MUST be zero for IKE (SPI is in message header) or four for $\mathrm{AH}$ and ESP.

- \# of SPIs (2 octets) - The number of SPIs contained in the Delete payload. The size of each SPI is defined by the SPI Size field.

- Security Parameter Index(es) (variable length) - Identifies the specific security association(s) to delete. The length of this field is determined by the SPI Size and \# of SPIs fields.

The payload type for the Delete Payload is forty two (42).

\subsection{Vendor ID Payload}

The Vendor ID Payload, denoted $V$ in this memo, contains a vendor defined constant. The constant is used by vendors to identify and recognize remote instances of their implementations. This mechanism allows a vendor to experiment with new features while maintaining backward compatibility.

A Vendor ID payload MAY announce that the sender is capable to accepting certain extensions to the protocol, or it MAY simply identify the implementation as an aid in debugging. A Vendor ID payload MUST NOT change the interpretation of any information defined in this specification (i.e., the critical bit MUST be set to 0 ). Multiple Vendor ID payloads MAY be sent. An implementation is NOT REQUIRED to send any Vendor ID payload at all.

A Vendor ID payload may be sent as part of any message. Reception of a familiar Vendor ID payload allows an implementation to make use of Private USE numbers described throughout this memo -- private payloads, private exchanges, private notifications, etc. Unfamiliar Vendor IDs MUST be ignored.

Writers of Internet-Drafts who wish to extend this protocol MUST define a Vendor ID payload to announce the ability to implement the extension in the Internet-Draft. It is expected that Internet-Drafts that gain acceptance and are standardized will be given "magic numbers" out of the Future Use range by IANA, and the requirement to use a Vendor ID will go away. 
The Vendor ID Payload fields are defined as follows:

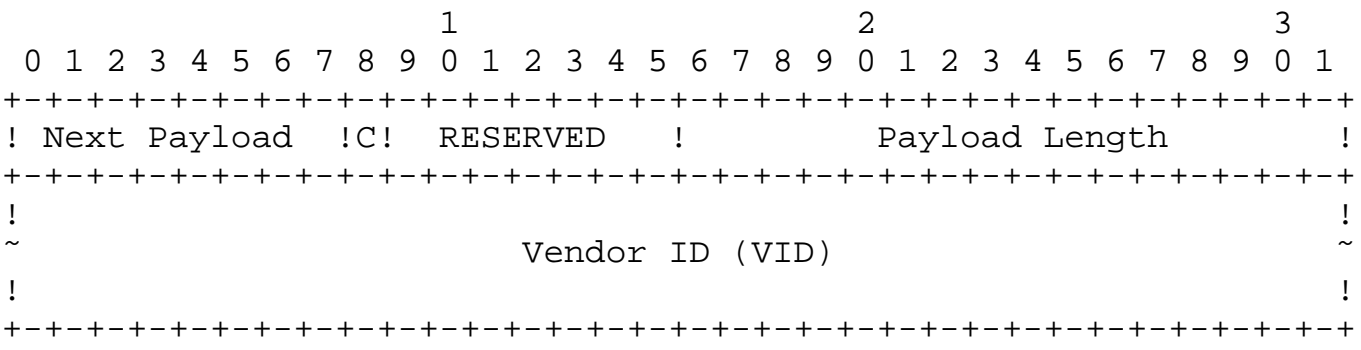

Figure 18: Vendor ID Payload Format

- Vendor ID (variable length) - It is the responsibility of the person choosing the Vendor ID to assure its uniqueness in spite of the absence of any central registry for IDs. Good practice is to include a company name, a person name, or some such. If you want to show off, you might include the latitude and longitude and time where you were when you chose the ID and some random input. A message digest of a long unique string is preferable to the long unique string itself.

The payload type for the Vendor ID Payload is forty three (43).

\subsection{Traffic Selector Payload}

The Traffic Selector Payload, denoted TS in this memo, allows peers to identify packet flows for processing by IPsec security services. The Traffic Selector Payload consists of the IKE generic payload header followed by individual traffic selectors as follows:

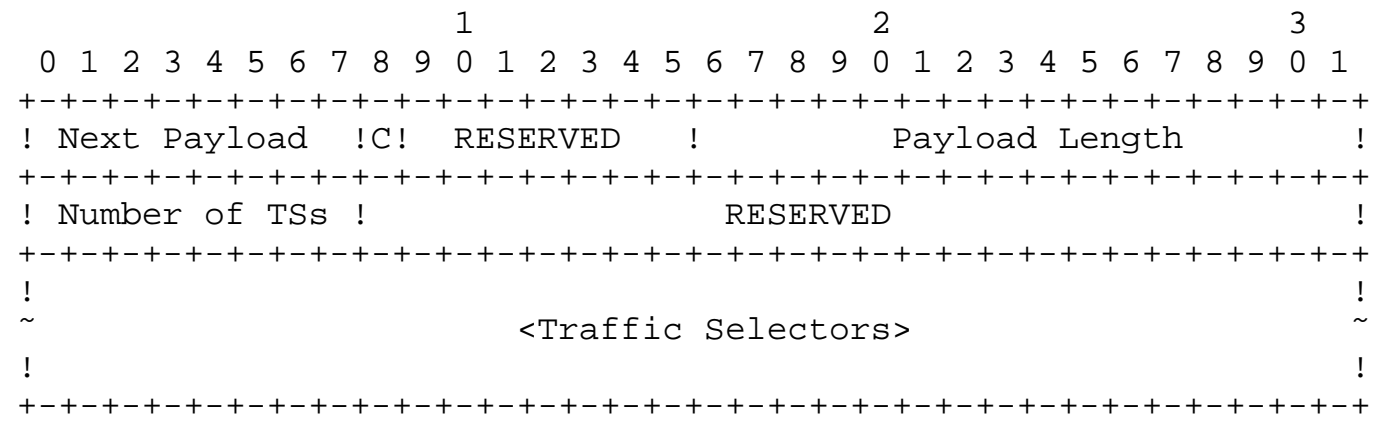

Figure 19: Traffic Selectors Payload Format

- Number of TSs (1 octet) - Number of traffic selectors being provided. 
- RESERVED - This field MUST be sent as zero and MUST be ignored on receipt.

- Traffic Selectors (variable length) - One or more individual traffic selectors.

The length of the Traffic Selector payload includes the TS header and all the traffic selectors.

The payload type for the Traffic selector payload is forty four (44) for addresses at the initiator's end of the SA and forty five (45) for addresses at the responder's end.

\subsubsection{Traffic Selector}

$\begin{array}{llllllllllllllll}1 & 2 & & & & \end{array}$

$\begin{array}{llllllllllllllllllllllllllllllll}0 & 1 & 2 & 3 & 4 & 5 & 6 & 7 & 8 & 9 & 0 & 1 & 2 & 3 & 4 & 5 & 6 & 7 & 8 & 9 & 0 & 1 & 2 & 3 & 4 & 5 & 6 & 7 & 8 & 9 & 0 & 1\end{array}$ +-+-+-+-+-+-+-+-+-+-+-+-+-+-+-+-+-+-+-+-+-+-+-+-+-+-+-+-+-+-+-+-t ! TS Type !IP Protocol ID* $\quad$ Selector Length $+-+-+-+-+-+-+-+-+-+-+-+-+-+-+-+-+-+-+-+-+-+-+-+-+-+-+-+-+-+-+-+-+$ Start Port* | End Port +-+-+-+-+-+-+-+-+-+-+-+-+-+-+-+-+-+-+-+-+-+-+-+-+-+-+-+-+-+-+-+-+ ! ! ! $+-+-+-+-+-+-+-+-+-+-+-+-+-+-+-+-+-+-+-+-+-+-+-+-+-+-+-+-+-+-+-+-+$ $\begin{array}{lll}\dot{\sim} & \text { Ending Address* } & \text { ! } \\ \text { ! } & & \text { ! }\end{array}$

Figure 20: Traffic Selector

* Note: All fields other than TS Type and Selector Length depend on the TS Type. The fields shown are for TS Types 7 and 8 , the only two values currently defined.

o TS Type (one octet) - Specifies the type of traffic selector.

- IP protocol ID (1 octet) - Value specifying an associated IP protocol ID (e.g., UDP/TCP/ICMP). A value of zero means that the protocol ID is not relevant to this traffic selector -- the SA can carry all protocols.

- Selector Length - Specifies the length of this Traffic Selector Substructure including the header. 
- Start Port (2 octets) - Value specifying the smallest port number allowed by this Traffic selector. For protocols for which port is undefined, or if all ports are allowed, this field MUST be zero. For the ICMP protocol, the two one-octet fields Type and Code are treated as a single 16-bit integer (with Type in the most significant eight bits and code in the least significant eight bits) port number for the purposes of filtering based on this field.

- End Port (2 octets) - Value specifying the largest port number allowed by this Traffic selector. For protocols for which port is undefined, or if all ports are allowed, this field MUST be 65535. For the ICMP protocol, the two one-octet fields Type and Code are treated as a single 16-bit integer (with Type in the most significant eight bits and code in the least significant eight bits) port number for the purposed of filtering based on this field.

- Starting Address - The smallest address included in this Traffic Selector (length determined by TS type).

- Ending Address - The largest address included in this Traffic Selector (length determined by TS type).

Systems that are complying with [RFC4301] that wish to indicate "ANY" ports MUST set the start port to 0 and the end port to 65535; note that according to [RFC4301], "ANY" includes "OPAQUE". Systems working with [RFC4301] that wish to indicate "OPAQUE" ports, but not "ANY" ports, MUST set the start port to 65535 and the end port to 0 .

The following table lists the assigned values for the Traffic Selector Type field and the corresponding Address Selector Data.

TS Type
$\begin{gathered}\text { Value } \\ ---- \\ \text { RESERVED } \\ 0-6\end{gathered}$
TS_IPV4_ADDR_RANGE
A range of IPv4 addresses, represented by two four-octet
values. The first value is the beginning IPv4 address
(inclusive) and the second value is the ending IPv4 address
(inclusive). All addresses falling between the two
specified addresses are considered to be within the list.


A range of IPv6 addresses, represented by two sixteen-octet values. The first value is the beginning IPv6 address (inclusive) and the second value is the ending IPv6 address (inclusive). All addresses falling between the two specified addresses are considered to be within the list.

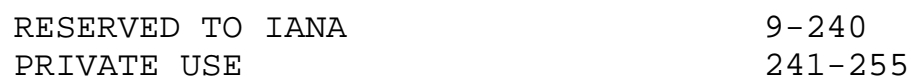

\subsection{Encrypted Payload}

The Encrypted Payload, denoted SK\{... or E in this memo, contains other payloads in encrypted form. The Encrypted Payload, if present in a message, MUST be the last payload in the message. Often, it is the only payload in the message.

The algorithms for encryption and integrity protection are negotiated during IKE_SA setup, and the keys are computed as specified in sections 2.14 and 2.18 .

The encryption and integrity protection algorithms are modeled after the ESP algorithms described in RFCs 2104 [KBC96], 4303 [RFC4303], and 2451 [ESPCBC]. This document completely specifies the cryptographic processing of IKE data, but those documents should be consulted for design rationale. We require a block cipher with a fixed block size and an integrity check algorithm that computes a fixed-length checksum over a variable size message.

The payload type for an Encrypted payload is forty six (46). The Encrypted Payload consists of the IKE generic payload header followed by individual fields as follows: 


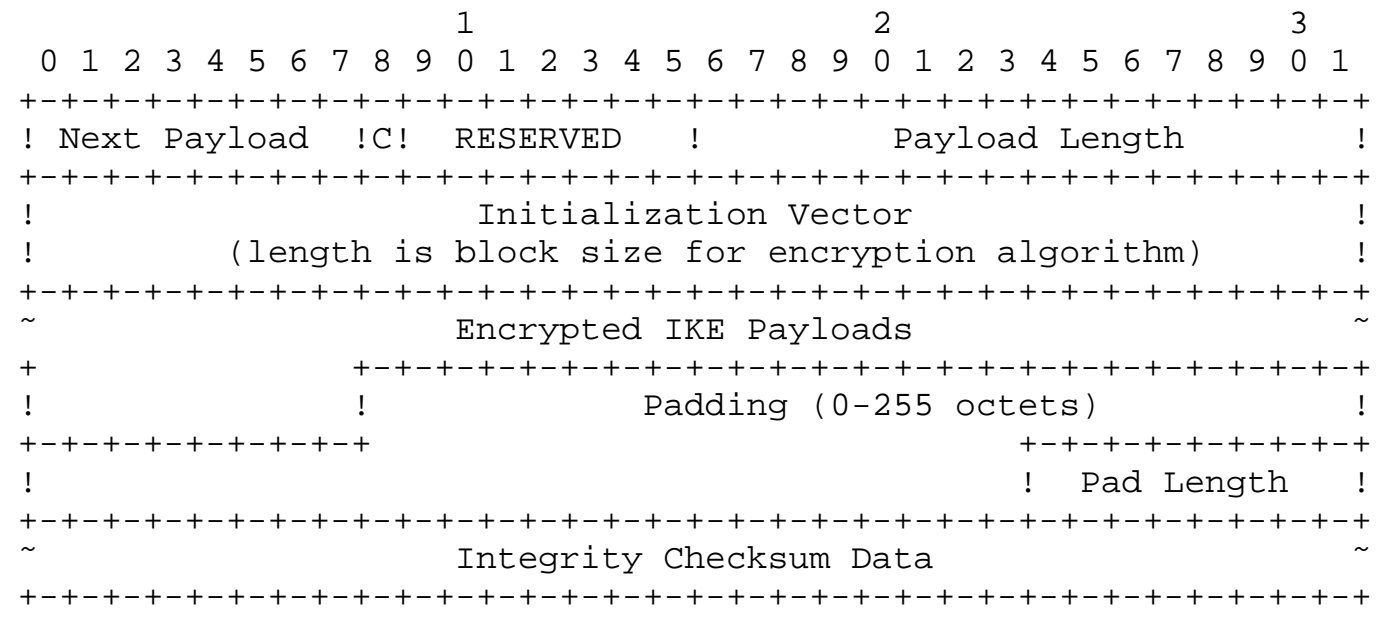

Figure 21: Encrypted Payload Format

- Next Payload - The payload type of the first embedded payload. Note that this is an exception in the standard header format, since the Encrypted payload is the last payload in the message and therefore the Next Payload field would normally be zero. But because the content of this payload is embedded payloads and there was no natural place to put the type of the first one, that type is placed here.

- Payload Length - Includes the lengths of the header, IV, Encrypted IKE Payloads, Padding, Pad Length, and Integrity Checksum Data.

- Initialization Vector - A randomly chosen value whose length is equal to the block length of the underlying encryption algorithm. Recipients MUST accept any value. Senders SHOULD either pick this value pseudo-randomly and independently for each message or use the final ciphertext block of the previous message sent. Senders MUST NOT use the same value for each message, use a sequence of values with low hamming distance (e.g., a sequence number), or use ciphertext from a received message.

- IKE Payloads are as specified earlier in this section. This field is encrypted with the negotiated cipher.

- Padding MAY contain any value chosen by the sender, and MUST have a length that makes the combination of the Payloads, the Padding, and the Pad Length to be a multiple of the encryption block size. This field is encrypted with the negotiated cipher. 
- Pad Length is the length of the Padding field. The sender SHOULD set the Pad Length to the minimum value that makes the combination of the Payloads, the Padding, and the Pad Length a multiple of the block size, but the recipient MUST accept any length that results in proper alignment. This field is encrypted with the negotiated cipher.

- Integrity Checksum Data is the cryptographic checksum of the entire message starting with the Fixed IKE Header through the Pad Length. The checksum MUST be computed over the encrypted message. Its length is determined by the integrity algorithm negotiated.

\subsection{Configuration Payload}

The Configuration payload, denoted CP in this document, is used to exchange configuration information between IKE peers. The exchange is for an IRAC to request an internal IP address from an IRAS and to exchange other information of the sort that one would acquire with Dynamic Host Configuration Protocol (DHCP) if the IRAC were directly connected to a LAN.

Configuration payloads are of type CFG_REQUEST/CFG_REPLY or CFG_SET/CFG_ACK (see CFG Type in the payload description below). CFG_REQUEST and CFG_SET payloads may optionally be added to any IKE request. The IKE response MUST include either a corresponding CFG_REPLY or CFG_ACK or a Notify payload with an error type indicating why the request could not be honored. An exception is that a minimal implementation MAY ignore all CFG_REQUEST and CFG_SET payloads, so a response message without a corresponding CFG_REPLY or CFG_ACK MUST be accepted as an indication that the request was not supported.

"CFG_REQUEST/CFG_REPLY" allows an IKE endpoint to request information from its peer. If an attribute in the CFG_REQUEST Configuration Payload is not zero-length, it is taken as a suggestion for that attribute. The CFG_REPLY Configuration Payload MAY return that value, or a new one. It MAY also add new attributes and not include some requested ones. Requestors MUST ignore returned attributes that they do not recognize.

Some attributes MAY be multi-valued, in which case multiple attribute values of the same type are sent and/or returned. Generally, all values of an attribute are returned when the attribute is requested. For some attributes (in this version of the specification only internal addresses), multiple requests indicates a request that multiple values be assigned. For these attributes, the number of values returned SHOULD NOT exceed the number requested. 
If the data type requested in a CFG_REQUEST is not recognized or not supported, the responder MUST NOT return an error type but rather MUST either send a CFG_REPLY that MAY be empty or a reply not containing a CFG_REPLY payload at all. Error returns are reserved for cases where the request is recognized but cannot be performed as requested or the request is badly formatted.

"CFG_SET/CFG_ACK" allows an IKE endpoint to push configuration data to its peer. In this case, the CFG_SET Configuration Payload contains attributes the initiator wants its peer to alter. The responder MUST return a Configuration Payload if it accepted any of the configuration data and it MUST contain the attributes that the responder accepted with zero-length data. Those attributes that it did not accept MUST NOT be in the CFG_ACK Configuration Payload. If no attributes were accepted, the responder MUST return either an empty CFG_ACK payload or a response message without a CFG_ACK payload. There are currently no defined uses for the CFG_SET/CFG_ACK exchange, though they may be used in connection with extensions based on Vendor IDs. An minimal implementation of this specification MAY ignore CFG_SET payloads.

Extensions via the CP payload SHOULD NOT be used for general purpose management. Its main intent is to provide a bootstrap mechanism to exchange information within IPsec from IRAS to IRAC. While it MAY be useful to use such a method to exchange information between some Security Gateways (SGW) or small networks, existing management protocols such as DHCP [DHCP], RADIUS [RADIUS], SNMP, or LDAP [LDAP] should be preferred for enterprise management as well as subsequent information exchanges.

The Configuration Payload is defined as follows:

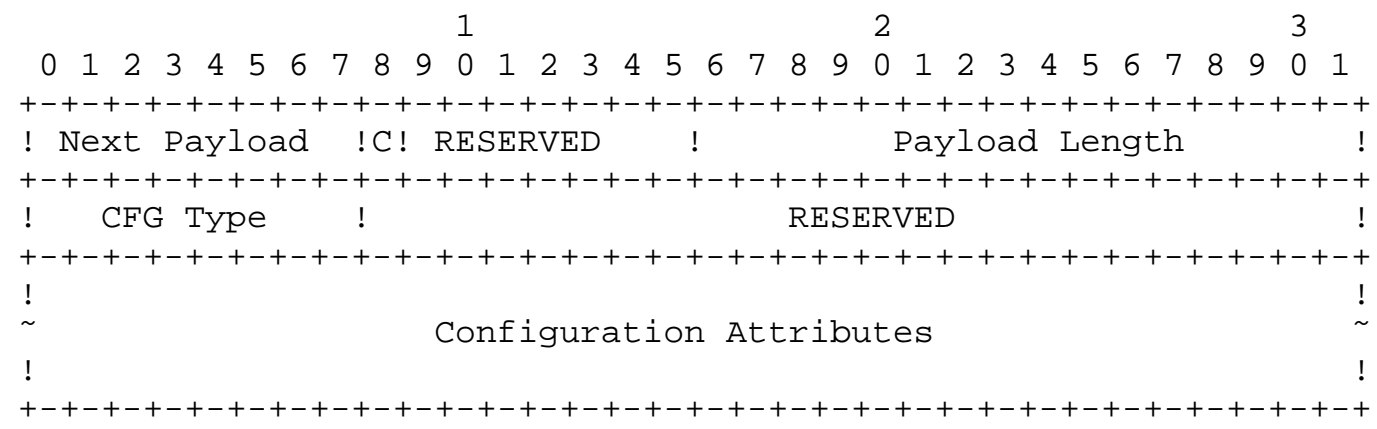

Figure 22: Configuration Payload Format

The payload type for the Configuration Payload is forty seven (47). 
- CFG Type (1 octet) - The type of exchange represented by the Configuration Attributes.

$\begin{array}{lc}\text { CFG Type } & \text { Value } \\ ========= & === \\ \text { RESERVED } & 0 \\ \text { CFG_REQUEST } & 1 \\ \text { CFG_REPLY } & 2 \\ \text { CFG_SET } & 3 \\ \text { CFG_ACK } & 4\end{array}$

values 5-127 are reserved to IANA. Values 128-255 are for private use among mutually consenting parties.

- RESERVED (3 octets) - MUST be sent as zero; MUST be ignored on receipt.

- Configuration Attributes (variable length) - These are type length values specific to the Configuration Payload and are defined below. There may be zero or more Configuration Attributes in this payload.

3.15.1. Configuration Attributes

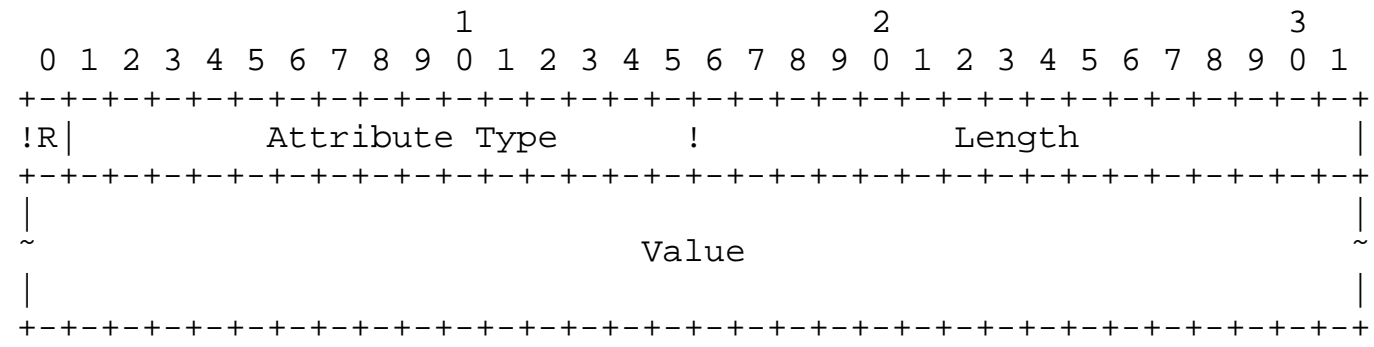

Figure 23: Configuration Attribute Format

- Reserved (1 bit) - This bit MUST be set to zero and MUST be ignored on receipt.

- Attribute Type (15 bits) - A unique identifier for each of the Configuration Attribute Types.

o Length (2 octets) - Length in octets of Value.

- Value (0 or more octets) - The variable-length value of this Configuration Attribute. 
The following attribute types have been defined:

\begin{tabular}{|c|c|c|c|}
\hline $\begin{array}{l}\text { Attribute Type } \\
===================\end{array}$ & $\begin{array}{l}\text { Value } \\
=====\end{array}$ & $\begin{array}{l}\text { Multi- } \\
\text { Valued } \\
======\end{array}$ & $\begin{array}{l}\text { Length } \\
================\end{array}$ \\
\hline RESERVED & 0 & & \\
\hline INTERNAL_IP 4_ADDRESS & 1 & YES * & 0 or 4 octets \\
\hline INTERNAL_IP 4_NETMASK & 2 & NO & 0 or 4 octets \\
\hline INTERNAL_IP4_DNS & 3 & YES & 0 or 4 octets \\
\hline INTERNAL_IP 4_NBNS & 4 & YES & 0 or 4 octets \\
\hline INTERNAL_ADDRESS_EXPIRY & 5 & NO & 0 or 4 octets \\
\hline INTERNAL_IP 4_DHCP & 6 & YES & 0 or 4 octets \\
\hline APPLICATION_VERSION & 7 & NO & 0 or more \\
\hline INTERNAL_IP 6_ADDRESS & 8 & YES* & 0 or 17 octets \\
\hline RESERVED & 9 & & \\
\hline INTERNAL_IP6_DNS & 10 & YES & 0 or 16 octets \\
\hline INTERNAL_IP6_NBNS & 11 & YES & 0 or 16 octets \\
\hline INTERNAL_IP 6_DHCP & 12 & YES & 0 or 16 octets \\
\hline INTERNAL_IP 4_SUBNET & 13 & YES & 0 or 8 octets \\
\hline SUPPORTED_ATTRIBUTES & 14 & NO & Multiple of 2 \\
\hline INTERNAL_IP 6_SUBNET & 15 & YES & 17 octets \\
\hline
\end{tabular}

* These attributes may be multi-valued on return only if multiple values were requested.

Types 16-16383 are reserved to IANA. Values 16384-32767 are for private use among mutually consenting parties.

O INTERNAL_IP4_ADDRESS, INTERNAL_IP6_ADDRESS - An address on the internal network, sometimes called a red node address or private address and MAY be a private address on the Internet. In a request message, the address specified is a requested address (or zero if no specific address is requested). If a specific address is requested, it likely indicates that a previous connection existed with this address and the requestor would like to reuse that address. With IPv6, a requestor MAY supply the low-order address bytes it wants to use. Multiple internal addresses MAY be requested by requesting multiple internal address attributes. The responder MAY only send up to the number of addresses requested. The INTERNAL_IP6_ADDRESS is made up of two fields: the first is a sixteen-octet IPv6 address and the second is a one-octet prefix-length as defined in [ADDRIPV6].

The requested address is valid until the expiry time defined with the INTERNAL_ADDRESS EXPIRY attribute or there are no IKE_SAs between the peers. 
- INTERNAL_IP4_NETMASK - The internal network's netmask. Only one netmask is allowed in the request and reply messages (e.g., 255.255.255.0), and it MUST be used only with an INTERNAL_IP4_ADDRESS attribute.

- INTERNAL_IP4_DNS, INTERNAL_IP6_DNS - Specifies an address of a DNS server within the network. Multiple DNS servers MAY be requested. The responder MAY respond with zero or more DNS server attributes.

- INTERNAL_IP4_NBNS, INTERNAL_IP6_NBNS - Specifies an address of a NetBios Name Server (WINS) within the network. Multiple NBNS servers MAY be requested. The responder MAY respond with zero or more NBNS server attributes.

- INTERNAL_ADDRESS_EXPIRY - Specifies the number of seconds that the host can use the internal IP address. The host MUST renew the IP address before this expiry time. Only one of these attributes MAY be present in the reply.

O INTERNAL_IP4_DHCP, INTERNAL_IP6_DHCP - Instructs the host to send any internal DHCP requests to the address contained within the attribute. Multiple DHCP servers MAY be requested. The responder MAY respond with zero or more DHCP server attributes.

- APPLICATION_VERSION - The version or application information of the IPsec host. This is a string of printable ASCII characters that is NOT null terminated.

- INTERNAL_IP4_SUBNET - The protected sub-networks that this edge-device protects. This attribute is made up of two fields: the first is an IP address and the second is a netmask. Multiple sub-networks MAY be requested. The responder MAY respond with zero or more sub-network attributes.

- SUPPORTED_ATTRIBUTES - When used within a Request, this attribute MUST be zero-length and specifies a query to the responder to reply back with all of the attributes that it supports. The response contains an attribute that contains a set of attribute identifiers each in 2 octets. The length divided by 2 (octets) would state the number of supported attributes contained in the response. 
O INTERNAL_IP6_SUBNET - The protected sub-networks that this edge-device protects. This attribute is made up of two fields: the first is a sixteen-octet IPv6 address and the second is a one-octet prefix-length as defined in [ADDRIPV6]. Multiple sub-networks MAY be requested. The responder MAY respond with zero or more sub-network attributes.

Note that no recommendations are made in this document as to how an implementation actually figures out what information to send in a reply. That is, we do not recommend any specific method of an IRAS determining which DNS server should be returned to a requesting IRAC.

\subsection{Extensible Authentication Protocol (EAP) Payload}

The Extensible Authentication Protocol Payload, denoted EAP in this memo, allows IKE_SAs to be authenticated using the protocol defined in RFC 3748 [EAP] and subsequent extensions to that protocol. The full set of acceptable values for the payload is defined elsewhere, but a short summary of RFC 3748 is included here to make this document stand alone in the common cases.

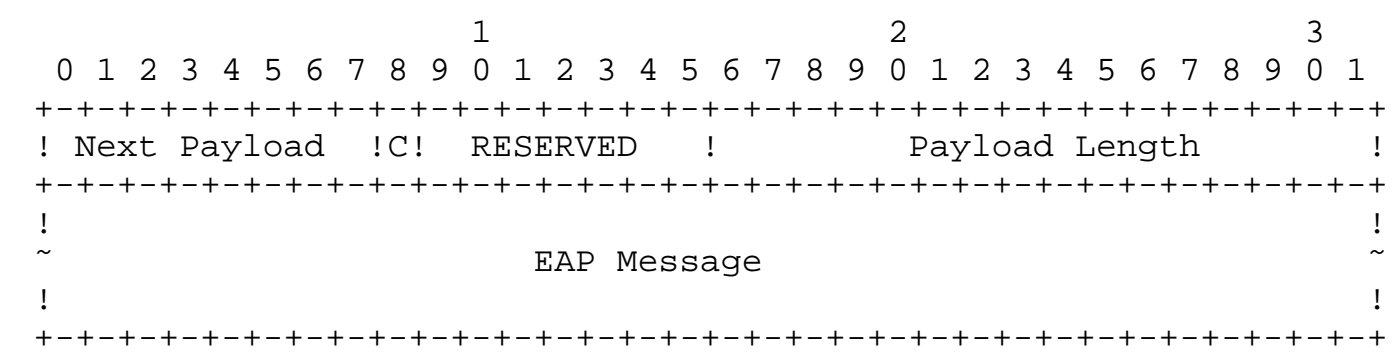

\section{Figure 24: EAP Payload Format}

The payload type for an EAP Payload is forty eight (48).

1

2

3

$\begin{array}{llllllllllllllllllllllllllllllll}0 & 1 & 2 & 3 & 4 & 5 & 6 & 7 & 8 & 9 & 0 & 1 & 2 & 3 & 4 & 5 & 6 & 7 & 8 & 9 & 0 & 1 & 2 & 3 & 4 & 5 & 6 & 7 & 8 & 9 & 0 & 1\end{array}$

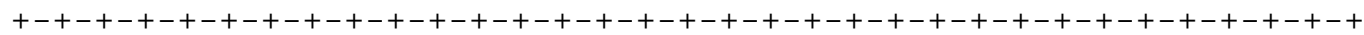
! Code ! Identifier ! Length +-+-+-+-+-+-+-+-+-+-+-+-+-+-+-+-+-+-+-+-+-+-+-+-+-+-+-+-+-+-+-+-+ ! Type ! Type_Data...

$+-+-+-+-+-+-+-+-+-+-+-+-+-+-+-+-+-+-+-+-$

Figure 25: EAP Message Format

- Code (1 octet) indicates whether this message is a Request (1), Response (2), Success (3), or Failure (4). 
- Identifier (1 octet) is used in PPP to distinguish replayed messages from repeated ones. Since in IKE, EAP runs over a reliable protocol, it serves no function here. In a response message, this octet MUST be set to match the identifier in the corresponding request. In other messages, this field MAY be set to any value.

- Length (2 octets) is the length of the EAP message and MUST be four less than the Payload Length of the encapsulating payload.

- Type (1 octet) is present only if the Code field is Request (1) or Response (2). For other codes, the EAP message length MUST be four octets and the Type and Type_Data fields MUST NOT be present. In a Request (1) message, Type indicates the data being requested. In a Response (2) message, Type MUST either be Nak or match the type of the data requested. The following types are defined in RFC 3748:

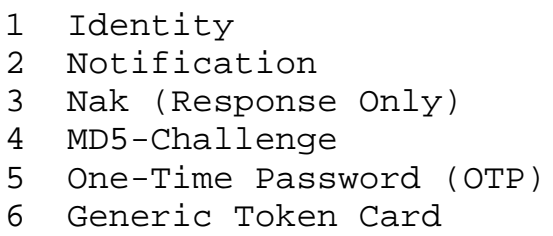

- Type_Data (Variable Length) varies with the Type of Request and the associated Response. For the documentation of the EAP methods, see [EAP].

Note that since IKE passes an indication of initiator identity in message 3 of the protocol, the responder SHOULD NOT send EAP Identity requests. The initiator SHOULD, however, respond to such requests if it receives them.

4. Conformance Requirements

In order to assure that all implementations of IKEv2 can interoperate, there are "MUST support" requirements in addition to those listed elsewhere. Of course, IKEv2 is a security protocol, and one of its major functions is to allow only authorized parties to successfully complete establishment of SAs. So a particular implementation may be configured with any of a number of restrictions concerning algorithms and trusted authorities that will prevent universal interoperability. 
IKEv2 is designed to permit minimal implementations that can interoperate with all compliant implementations. There are a series of optional features that can easily be ignored by a particular implementation if it does not support that feature. Those features include:

Ability to negotiate SAs through a NAT and tunnel the resulting ESP SA over UDP.

Ability to request (and respond to a request for) a temporary IP address on the remote end of a tunnel.

Ability to support various types of legacy authentication.

Ability to support window sizes greater than one.

Ability to establish multiple ESP and/or AH SAs within a single IKE_SA.

Ability to rekey SAs.

To assure interoperability, all implementations MUST be capable of parsing all payload types (if only to skip over them) and to ignore payload types that it does not support unless the critical bit is set in the payload header. If the critical bit is set in an unsupported payload header, all implementations MUST reject the messages containing those payloads.

Every implementation MUST be capable of doing four-message IKE_SA_INIT and IKE_AUTH exchanges establishing two SAs (one for IKE, one for ESP and/or AH). Implementations MAY be initiate-only or respond-only if appropriate for their platform. Every implementation MUST be capable of responding to an INFORMATIONAL exchange, but a minimal implementation MAY respond to any INFORMATIONAL message with an empty INFORMATIONAL reply (note that within the context of an IKE_SA, an "empty" message consists of an IKE header followed by an Encrypted payload with no payloads contained in it). A minimal implementation MAY support the CREATE_CHILD_SA exchange only in so far as to recognize requests and reject them with a Notify payload of type NO_ADDITIONAL_SAS. A minimal implementation need not be able to initiate CREATE_CHILD_SA or INFORMATIONAL exchanges. When an SA expires (based on locally configured values of either lifetime or octets passed), and implementation MAY either try to renew it with a CREATE_CHILD_SA exchange or it MAY delete (close) the old SA and create a new one. If the responder rejects the CREATE_CHILD_SA request with a NO_ADDITIONAL_SAS notification, the implementation MUST be capable of instead closing the old SA and creating a new one. 
Implementations are not required to support requesting temporary IP addresses or responding to such requests. If an implementation does support issuing such requests, it MUST include a CP payload in message 3 containing at least a field of type INTERNAL_IP4_ADDRESS or INTERNAL_IP6_ADDRESS. All other fields are optional. If an implementation supports responding to such requests, it MUST parse the CP payload of type CFG_REQUEST in message 3 and recognize a field of type INTERNAL_IP4_ADDRESS or INTERNAL_IP6_ADDRESS. If it supports leasing an address of the appropriate type, it MUST return a CP payload of type CFG_REPLY containing an address of the requested type. The responder SHOULD include all of the other related attributes if it has them.

A minimal IPv4 responder implementation will ignore the contents of the CP payload except to determine that it includes an INTERNAL_IP4_ADDRESS attribute and will respond with the address and other related attributes regardless of whether the initiator requested them.

A minimal IPv4 initiator will generate a CP payload containing only an INTERNAL_IP4_ADDRESS attribute and will parse the response ignoring attributes it does not know how to use. The only attribute it MUST be able to process is INTERNAL_ADDRESS_EXPIRY, which it must use to bound the lifetime of the SA unless it successfully renews the lease before it expires. Minimal initiators need not be able to request lease renewals and minimal responders need not respond to them.

For an implementation to be called conforming to this specification, it MUST be possible to configure it to accept the following:

PKIX Certificates containing and signed by RSA keys of size 1024 or 2048 bits, where the ID passed is any of ID_KEY_ID, ID_FQDN, ID_RFC822_ADDR, or ID_DER_ASN1_DN.

Shared key authentication where the ID passes is any of ID_KEY_ID, ID_FQDN, or ID_RFC822_ADDR.

Authentication where the responder is authenticated using PKIX Certificates and the initiator is authenticated using shared key authentication. 


\section{Security Considerations}

While this protocol is designed to minimize disclosure of configuration information to unauthenticated peers, some such disclosure is unavoidable. One peer or the other must identify itself first and prove its identity first. To avoid probing, the initiator of an exchange is required to identify itself first, and usually is required to authenticate itself first. The initiator can, however, learn that the responder supports IKE and what cryptographic protocols it supports. The responder (or someone impersonating the responder) can probe the initiator not only for its identity, but using CERTREQ payloads may be able to determine what certificates the initiator is willing to use.

Use of EAP authentication changes the probing possibilities somewhat. When EAP authentication is used, the responder proves its identity before the initiator does, so an initiator that knew the name of a valid initiator could probe the responder for both its name and certificates.

Repeated rekeying using CREATE_CHILD_SA without additional DiffieHellman exchanges leaves all SAs vulnerable to cryptanalysis of a single key or overrun of either endpoint. Implementers should take note of this fact and set a limit on CREATE_CHILD_SA exchanges between exponentiations. This memo does not prescribe such a limit.

The strength of a key derived from a Diffie-Hellman exchange using any of the groups defined here depends on the inherent strength of the group, the size of the exponent used, and the entropy provided by the random number generator used. Due to these inputs, it is difficult to determine the strength of a key for any of the defined groups. Diffie-Hellman group number two, when used with a strong random number generator and an exponent no less than 200 bits, is common for use with 3DES. Group five provides greater security than group two. Group one is for historic purposes only and does not provide sufficient strength except for use with DES, which is also for historic use only. Implementations should make note of these estimates when establishing policy and negotiating security parameters.

Note that these limitations are on the Diffie-Hellman groups themselves. There is nothing in IKE that prohibits using stronger groups nor is there anything that will dilute the strength obtained from stronger groups (limited by the strength of the other algorithms negotiated including the prf function). In fact, the extensible framework of IKE encourages the definition of more groups; use of elliptical curve groups may greatly increase strength using much smaller numbers. 
It is assumed that all Diffie-Hellman exponents are erased from memory after use. In particular, these exponents MUST NOT be derived from long-lived secrets like the seed to a pseudo-random generator that is not erased after use.

The strength of all keys is limited by the size of the output of the negotiated prf function. For this reason, a prf function whose output is less than 128 bits (e.g., 3DES-CBC) MUST NOT be used with this protocol.

The security of this protocol is critically dependent on the randomness of the randomly chosen parameters. These should be generated by a strong random or properly seeded pseudo-random source (see [RFC4086]). Implementers should take care to ensure that use of random numbers for both keys and nonces is engineered in a fashion that does not undermine the security of the keys.

For information on the rationale of many of the cryptographic design choices in this protocol, see [SIGMA] and [SKEME]. Though the security of negotiated CHILD_SAs does not depend on the strength of the encryption and integrity protection negotiated in the IKE_SA, implementations MUST NOT negotiate NONE as the IKE integrity protection algorithm or ENCR_NULL as the IKE encryption algorithm.

When using pre-shared keys, a critical consideration is how to assure the randomness of these secrets. The strongest practice is to ensure that any pre-shared key contain as much randomness as the strongest key being negotiated. Deriving a shared secret from a password, name, or other low-entropy source is not secure. These sources are subject to dictionary and social engineering attacks, among others.

The NAT_DETECTION_*_IP notifications contain a hash of the addresses and ports in an attempt to hide internal IP addresses behind a NAT. Since the IPv4 address space is only 32 bits, and it is usually very sparse, it would be possible for an attacker to find out the internal address used behind the NAT box by trying all possible IP addresses and trying to find the matching hash. The port numbers are normally fixed to 500, and the SPIs can be extracted from the packet. This reduces the number of hash calculations to $2^{\wedge} 32$. With an educated guess of the use of private address space, the number of hash calculations is much smaller. Designers should therefore not assume that use of IKE will not leak internal address information.

When using an EAP authentication method that does not generate a shared key for protecting a subsequent AUTH payload, certain man-inthe-middle and server impersonation attacks are possible [EAPMITM]. These vulnerabilities occur when EAP is also used in protocols that are not protected with a secure tunnel. Since EAP is a general- 
purpose authentication protocol, which is often used to provide single-signon facilities, a deployed IPsec solution that relies on an EAP authentication method that does not generate a shared key (also known as a non-key-generating EAP method) can become compromised due to the deployment of an entirely unrelated application that also happens to use the same non-key-generating EAP method, but in an unprotected fashion. Note that this vulnerability is not limited to just EAP, but can occur in other scenarios where an authentication infrastructure is reused. For example, if the EAP mechanism used by IKEv2 utilizes a token authenticator, a man-in-the-middle attacker could impersonate the web server, intercept the token authentication exchange, and use it to initiate an IKEv2 connection. For this reason, use of non-key-generating EAP methods SHOULD be avoided where possible. Where they are used, it is extremely important that all usages of these EAP methods SHOULD utilize a protected tunnel, where the initiator validates the responder's certificate before initiating the EAP exchange. Implementers SHOULD describe the vulnerabilities of using non-key-generating EAP methods in the documentation of their implementations so that the administrators deploying IPsec solutions are aware of these dangers.

An implementation using EAP MUST also use a public-key-based authentication of the server to the client before the EAP exchange begins, even if the EAP method offers mutual authentication. This avoids having additional IKEv2 protocol variations and protects the EAP data from active attackers.

If the messages of IKEv2 are long enough that IP-level fragmentation is necessary, it is possible that attackers could prevent the exchange from completing by exhausting the reassembly buffers. The chances of this can be minimized by using the Hash and URL encodings instead of sending certificates (see section 3.6). Additional mitigations are discussed in [KPSO3].

\section{IANA Considerations}

This document defines a number of new field types and values where future assignments will be managed by the IANA.

The following registries have been created by the IANA:

IKEv2 Exchange Types (section 3.1)

IKEv2 Payload Types (section 3.2)

IKEv2 Transform Types (section 3.3.2)

IKEv2 Transform Attribute Types (section 3.3.2)

IKEv2 Encryption Transform IDs (section 3.3.2)

IKEv2 Pseudo-random Function Transform IDs (section 3.3.2)

IKEv2 Integrity Algorithm Transform IDs (section 3.3.2) 
IKEv2 Diffie-Hellman Transform IDs (section 3.3.2)

IKEv2 Identification Payload ID Types (section 3.5)

IKEv2 Certificate Encodings (section 3.6)

IKEv2 Authentication Method (section 3.8)

IKEv2 Notify Message Types (section 3.10.1)

IKEv2 Notification IPCOMP Transform IDs (section 3.10.1)

IKEv2 Security Protocol Identifiers (section 3.3.1)

IKEv2 Traffic Selector Types (section 3.13.1)

IKEv2 Configuration Payload CFG Types (section 3.15)

IKEv2 Configuration Payload Attribute Types (section 3.15.1)

Note: When creating a new Transform Type, a new registry for it must be created.

Changes and additions to any of those registries are by expert review.

\section{Acknowledgements}

This document is a collaborative effort of the entire IPsec WG. If there were no limit to the number of authors that could appear on an RFC, the following, in alphabetical order, would have been listed: Bill Aiello, Stephane Beaulieu, Steve Bellovin, Sara Bitan, Matt Blaze, Ran Canetti, Darren Dukes, Dan Harkins, Paul Hoffman, John Ioannidis, Charlie Kaufman, Steve Kent, Angelos Keromytis, Tero Kivinen, Hugo Krawczyk, Andrew Krywaniuk, Radia Perlman, Omer Reingold, and Michael Richardson. Many other people contributed to the design. It is an evolution of IKEV1, ISAKMP, and the IPsec DOI, each of which has its own list of authors. Hugh Daniel suggested the feature of having the initiator, in message 3, specify a name for the responder, and gave the feature the cute name "You Tarzan, Me Jane". David Faucher and Valery Smyzlov helped refine the design of the traffic selector negotiation.

8. References

8.1. Normative References

[ADDGROUP] Kivinen, T. and M. Kojo, "More Modular Exponential (MODP) Diffie-Hellman groups for Internet Key Exchange (IKE)", RFC 3526, May 2003.

[ADDRIPV6] Hinden, R. and S. Deering, "Internet Protocol Version 6 (IPv6) Addressing Architecture", RFC 3513, April 2003.

[Bra97] Bradner, S., "Key Words for use in RFCs to indicate Requirement Levels", BCP 14, RFC 2119, March 1997. 
[EAP ] Aboba, B., Blunk, L., Vollbrecht, J., Carlson, J., and H. Levkowetz, "Extensible Authentication Protocol (EAP)", RFC 3748 , June 2004 .

[ESPCBC] Pereira, R. and R. Adams, "The ESP CBC-Mode Cipher Algorithms", RFC 2451, November 1998.

[Hutt05] Huttunen, A., Swander, B., Volpe, V., DiBurro, L., and M. Stenberg, "UDP Encapsulation of IPsec ESP Packets", RFC 3948, January 2005 .

[RFC2434] Narten, T. and H. Alvestrand, "Guidelines for Writing an IANA Considerations Section in RFCs", BCP 26, RFC 2434, October 1998 .

[RFC3168] Ramakrishnan, K., Floyd, S., and D. Black, "The Addition of Explicit Congestion Notification (ECN) to IP", RFC 3168, September 2001 .

[RFC3280] Housley, R., Polk, W., Ford, W., and D. Solo, "Internet X.509 Public Key Infrastructure Certificate and Certificate Revocation List (CRL) Profile", RFC 3280, April 2002 .

[RFC4301] Kent, S. and K. Seo, "Security Architecture for the Internet Protocol", RFC 4301, December 2005.

8.2. Informative References

[DES] ANSI X3.106, "American National Standard for Information Systems-Data Link Encryption", American National Standards Institute, 1983 .

[DH] Diffie, W., and Hellman M., "New Directions in Cryptography", IEEE Transactions on Information Theory, V. $\mathrm{IT}-22$, n. 6, June 1977.

[DHCP] Droms, R., "Dynamic Host Configuration Protocol", RFC 2131, March 1997.

[DSS] NIST, "Digital Signature Standard", FIPS 186, National Institute of Standards and Technology, U.S. Department of Commerce, May, 1994.

[EAPMITM] Asokan, N., Nierni, V., and Nyberg, K., "Man-in-the-Middle in Tunneled Authentication Protocols", http://eprint.iacr.org/2002/163, November 2002 . 
[HC98] Harkins, D. and D. Carrel, "The Internet Key Exchange (IKE) ", RFC 2409, November 1998.

[IDEA] Lai, X., "On the Design and Security of Block Ciphers," ETH Series in Information Processing, $v \cdot 1$, Konstanz: Hartung-Gorre Verlag, 1992.

[IPCOMP] Shacham, A., Monsour, B., Pereira, R., and M. Thomas, "IP Payload Compression Protocol (IPComp)", RFC 3173, September 2001 .

[KPS03] Kaufman, C., Perlman, R., and Sommerfeld, B., "DoS protection for UDP-based protocols", ACM Conference on Computer and Communications Security, October 2003.

[KBC96] Krawczyk, H., Bellare, M., and R. Canetti, "HMAC: KeyedHashing for Message Authentication", RFC 2104, February 1997.

[LDAP] Wahl, M., Howes, T., and S Kille, "Lightweight Directory Access Protocol (v3)", RFC 2251, December 1997.

[MD5] Rivest, R., "The MD5 Message-Digest Algorithm", RFC 1321, April 1992 .

[MSST98] Maughan, D., Schertler, M., Schneider, M., and J. Turner, "Internet Security Association and Key Management Protocol (ISAKMP) ", RFC 2408, November 1998.

[Orm96] Orman, H., "The OAKLEY Key Determination Protocol", RFC 2412, November 1998 .

[PFKEY] McDonald, D., Metz, C., and B. Phan, "PF_KEY Key Management API, Version 2", RFC 2367, July 1998.

[PKCS1] Jonsson, J. and B. Kaliski, "Public-Key Cryptography Standards (PKCS) \#1: RSA Cryptography Specifications Version 2.1", RFC 3447, February 2003.

[PK01] Perlman, R., and Kaufman, C., "Analysis of the IPsec key exchange Standard", WET-ICE Security Conference, MIT,2001, http://sec.femto.org/wetice-2001/papers/radia-paper.pdf.

[Pip98] Piper, D., "The Internet IP Security Domain of Interpretation for ISAKMP", RFC 2407, November 1998. 
[RADIUS] Rigney, C., Willens, S., Rubens, A., and W. Simpson, "Remote Authentication Dial In User Service (RADIUS)", RFC 2865, June 2000 .

[RFC4086] Eastlake, D., 3rd, Schiller, J., and S. Crocker, "Randomness Requirements for Security", BCP 106, RFC 4086, June 2005.

[RFC1958] Carpenter, B., "Architectural Principles of the Internet", RFC 1958, June 1996.

[RFC2401] Kent, S. and R. Atkinson, "Security Architecture for the Internet Protocol", RFC 2401, November 1998.

[RFC2474] Nichols, K., Blake, S., Baker, F., and D. Black, "Definition of the Differentiated Services Field (DS Field) in the IPv4 and IPv6 Headers", RFC 2474, December 1998 .

[RFC2475] Blake, S., Black, D., Carlson, M., Davies, E., Wang, Z., and W. Weiss, "An Architecture for Differentiated Service", RFC 2475, December 1998.

[RFC2522] Karn, P. and W. Simpson, "Photuris: Session-Key Management Protocol", RFC 2522, March 1999.

[RFC2775] Carpenter, B., "Internet Transparency", RFC 2775, February 2000 .

[RFC2983] Black, D., "Differentiated Services and Tunnels", RFC 2983, October 2000 .

[RFC3439] Bush, R. and D. Meyer, "Some Internet Architectural Guidelines and Philosophy", RFC 3439, December 2002.

[RFC3715] Aboba, B. and W. Dixon, "IPsec-Network Address Translation (NAT) Compatibility Requirements", RFC 3715, March 2004.

[RFC4302] Kent, S., "IP Authentication Header", RFC 4302, December 2005 .

[RFC4303] Kent, S., "IP Encapsulating Security Payload (ESP) ", RFC 4303, December 2005.

[RSA] Rivest, R., Shamir, A., and Adleman, L., "A Method for Obtaining Digital Signatures and Public-Key Cryptosystems", Communications of the ACM, v. 21, n. 2, February 1978 . 
[SHA] NIST, "Secure Hash Standard", FIPS 180-1, National Institute of Standards and Technology, U.S. Department of Commerce, May 1994.

[SIGMA] Krawczyk, H., "SIGMA: the 'SIGn-and-MAc' Approach to Authenticated Diffie-Hellman and its Use in the IKE Protocols", in Advances in Cryptography - CRYPTO 2003 Proceedings, LNCS 2729, Springer, 2003. Available at: http://www.informatik.uni-trier.de/ ley/db/conf/ crypto/crypto2003.html.

[SKEME] Krawczyk, H., "SKEME: A Versatile Secure Key Exchange Mechanism for Internet", from IEEE Proceedings of the 1996 Symposium on Network and Distributed Systems Security.

[X.501] ITU-T Recommendation X.501: Information Technology - Open Systems Interconnection - The Directory: Models, 1993.

[X.509] ITU-T Recommendation X.509 (1997 E): Information Technology - Open Systems Interconnection - The Directory: Authentication Framework, June 1997. 
Appendix A: Summary of changes from IKEv1

The goals of this revision to IKE are:

1) To define the entire IKE protocol in a single document, replacing RFCs 2407, 2408, and 2409 and incorporating subsequent changes to support NAT Traversal, Extensible Authentication, and Remote Address acquisition;

2) To simplify IKE by replacing the eight different initial exchanges with a single four-message exchange (with changes in authentication mechanisms affecting only a single AUTH payload rather than restructuring the entire exchange) see [PKO1];

3) To remove the Domain of Interpretation (DOI), Situation (SIT), and Labeled Domain Identifier fields, and the Commit and Authentication only bits;

4) To decrease IKE's latency in the common case by making the initial exchange be 2 round trips (4 messages), and allowing the ability to piggyback setup of a CHILD_SA on that exchange;

5) To replace the cryptographic syntax for protecting the IKE messages themselves with one based closely on EsP to simplify implementation and security analysis;

6) To reduce the number of possible error states by making the protocol reliable (all messages are acknowledged) and sequenced. This allows shortening CREATE_CHILD_SA exchanges from 3 messages to 2 ;

7) To increase robustness by allowing the responder to not do significant processing until it receives a message proving that the initiator can receive messages at its claimed IP address, and not commit any state to an exchange until the initiator can be cryptographically authenticated;

8) To fix cryptographic weaknesses such as the problem with symmetries in hashes used for authentication documented by Tero Kivinen;

9) To specify Traffic Selectors in their own payloads type rather than overloading ID payloads, and making more flexible the Traffic Selectors that may be specified;

10) To specify required behavior under certain error conditions or when data that is not understood is received, to make it easier to make future revisions that do not break backward compatibility; 
11) To simplify and clarify how shared state is maintained in the presence of network failures and Denial of Service attacks; and

12) To maintain existing syntax and magic numbers to the extent possible to make it likely that implementations of IKEvl can be enhanced to support IKEv2 with minimum effort.

Appendix B: Diffie-Hellman Groups

There are two Diffie-Hellman groups defined here for use in IKE. These groups were generated by Richard Schroeppel at the University of Arizona. Properties of these primes are described in [Orm96].

The strength supplied by group one may not be sufficient for the mandatory-to-implement encryption algorithm and is here for historic reasons.

Additional Diffie-Hellman groups have been defined in [ADDGROUP].

B.1. Group $1-768$ Bit MODP

This group is assigned id 1 (one).

The prime is: $2^{\wedge} 768-2 \wedge 704-1+2^{\wedge} 64 \star\left\{\left[2^{\wedge} 638 \mathrm{pi}\right]+149686\right\}$ Its hexadecimal value is:

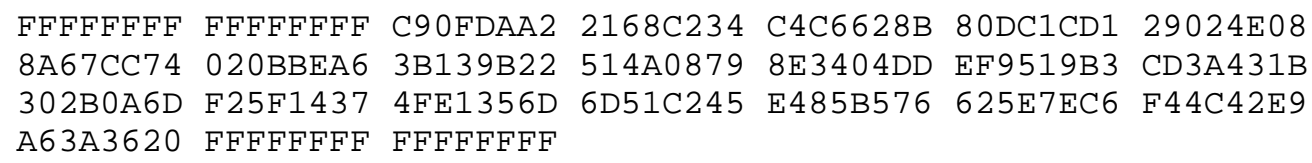

The generator is 2 .

B.2. Group 2 - 1024 Bit MODP

This group is assigned id 2 (two).

The prime is $2^{\wedge} 1024-2^{\wedge} 960-1+2^{\wedge} 64 \star\left\{\left[2^{\wedge} 894 \mathrm{pi}\right]+129093\right\}$. Its hexadecimal value is:

FFFFFFFF FFFFFFFF C90FDAA2 2168C234 C4C6628B 80DC1CD1 $29024 \mathrm{E} 08$

8A67CC74 020BBEA6 3B139B22 514A0879 8E3404DD EF9519B3 CD3A431B

302B0A6D F25F1437 4FE1356D 6D51C245 E485B576 625E7EC6 F44C42E9

A637ED6B OBFF5CB6 F406B7ED EE386BFB 5A899FA5 AE9F2411 7C4B1FE6

49286651 ECE65381 FFFFFFFF FFFFFFFF

The generator is 2 . 


\section{Editor's Address}

Charlie Kaufman

Microsoft Corporation

1 Microsoft Way

Redmond, WA 98052

Phone: 1-425-707-3335

EMail: charliek@microsoft.com 
Full Copyright statement

Copyright (C) The Internet Society (2005).

This document is subject to the rights, licenses and restrictions contained in BCP 78, and except as set forth therein, the authors retain all their rights.

This document and the information contained herein are provided on an "AS IS" basis and THE CONTRIBUTOR, THE ORGANIZATION HE/SHE REPRESENTS OR IS SPONSORED BY (IF ANY), THE INTERNET SOCIETY AND THE INTERNET ENGINEERING TASK FORCE DISCLAIM ALL WARRANTIES, EXPRESS OR IMPLIED, INCLUDING BUT NOT LIMITED TO ANY WARRANTY THAT THE USE OF THE INFORMATION HEREIN WILL NOT INFRINGE ANY RIGHTS OR ANY IMPLIED WARRANTIES OF MERCHANTABILITY OR FITNESS FOR A PARTICULAR PURPOSE.

Intellectual Property

The IETF takes no position regarding the validity or scope of any Intellectual Property Rights or other rights that might be claimed to pertain to the implementation or use of the technology described in this document or the extent to which any license under such rights might or might not be available; nor does it represent that it has made any independent effort to identify any such rights. Information on the procedures with respect to rights in RFC documents can be found in BCP 78 and BCP 79 .

Copies of IPR disclosures made to the IETF Secretariat and any assurances of licenses to be made available, or the result of an attempt made to obtain a general license or permission for the use of such proprietary rights by implementers or users of this specification can be obtained from the IETF on-line IPR repository at http://www.ietf.org/ipr.

The IETF invites any interested party to bring to its attention any copyrights, patents or patent applications, or other proprietary rights that may cover technology that may be required to implement this standard. Please address the information to the IETF at ietfipreietf.org.

Acknowledgement

Funding for the RFC Editor function is currently provided by the Internet Society. 